\title{
Localization and non-renormalization in Chern-Simons theory
}

\author{
Yale Fan \\ Department of Physics, Princeton University, \\ Princeton, NJ 08544, U.S.A. \\ E-mail: yalefan@gmail.com
}

ABSTRACT: We revisit and clarify some aspects of perturbative renormalization in pure Chern-Simons theory by means of a localization principle associated with an underlying supersymmetry. This perspective allows the otherwise perturbative one-loop shifts to be interpreted as nonperturbative consequences of a non-renormalization theorem, while providing a unified understanding of their origin (particularly in the case of Wilson lines). We illustrate this approach explicitly for SU(2) Chern-Simons theory in flat space, on Seifert manifolds, and on a solid torus.

KeYwords: Chern-Simons Theories, Supersymmetric Gauge Theory, Topological Field Theories

ARXIV EPRINT: 1805.11076 


\section{Contents}

1 Introduction 1

1.1 Old perspectives 2

1.2 New perspectives 3

$2 \mathcal{N}=0$ Chern-Simons theory $\quad 5$

2.1 Perturbation theory 6

2.2 Beyond perturbation theory 8

$\begin{array}{lll}3 & \text { Wilson loops and coadjoint orbits } & 9\end{array}$

3.1 The orbit method 9

$\begin{array}{lll}3.2 & \text { Wilson/'t Hooft loops in Chern-Simons theory } & 11\end{array}$

4 Wilson loops in $\mathcal{N}=2$ Chern-Simons theory 12

$\begin{array}{ll}\text { 4.1 Shift from line dynamics } & 12\end{array}$

$\begin{array}{lll}4.1 .1 \mathcal{N}=2 \text { coadjoint orbit } & 12\end{array}$

$\begin{array}{lll}\text { 4.1.2 Effective action } & 15\end{array}$

$\begin{array}{lll}4.2 & \text { Shift from canonical quantization } & 16\end{array}$

$\begin{array}{lll}\text { 4.2.1 } & \text { Bosonic system } & 17\end{array}$

$\begin{array}{lll}\text { 4.2.2 Supersymmetric system } & 17\end{array}$

$\begin{array}{lll}\text { 4.3 Shift from 1D supersymmetric index } & 18\end{array}$

$\begin{array}{lll}\text { 4.3.1 Localization in 1D } & 21\end{array}$

$\begin{array}{lll}4.3 .2 & \text { Finite temperature } & 22\end{array}$

$\begin{array}{ll}\text { 4.3.3 } & \text { Background gauge field }\end{array}$

5 Coupling to the bulk $\quad 25$

5.1 Linearly realized SUSY on the line 26

5.2 Nonlinearly realized SUSY on the line 26

$\begin{array}{lll}5.3 & \text { Nonlinearly realized SUSY in the sigma model } & 29\end{array}$

6 Localization in 3D $\quad 30$

6.1 Overview 30

$\begin{array}{lll}6.2 & \text { Supergravity background } & 31\end{array}$

6.3 Localizing "Seifert" loops 33

6.3.1 Example: $S^{3} \quad 36$

6.3.2 Example: $S_{b}^{3} \quad 38$

6.3.3 Non-example: (squashed) lens spaces 40

6.4 Localization on solid torus 41

7 Matching $\mathcal{N}=0$ and $\mathcal{N}=2$ line operators $\quad 44$

$\begin{array}{lll}7.1 & \text { (Non-)renormalization } & 44\end{array}$

$\begin{array}{ll}7.2 & 3 \mathrm{D} \text { point of view }\end{array}$

7.3 A quasi-2D point of view $\quad 45$

$\begin{array}{lll}8 & \text { Discussion } & 47\end{array}$ 
A.1 Gauge transformations 48

A.2 $1 \mathrm{D} \mathcal{N}=2 \quad 48$

A.3 $3 \mathrm{D} \mathcal{N}=2 \quad 50$

$\begin{array}{lll}\text { A.3.1 Lorentzian } & 50\end{array}$

$\begin{array}{lll}\text { A.3.2 Euclidean } & 51\end{array}$

B Gauging SUSY NLSMs

B.1 Kähler potential $\quad 55$

$\begin{array}{lll}\text { B.2 Higher-derivative terms } & 57\end{array}$

C Details on the $\mathcal{N}=2$ coadjoint orbit $\quad 57$

$\begin{array}{lll}\text { C.1 } & \mathbb{C P}^{1} \text { sigma model } & 57\end{array}$

$\begin{array}{lll}\text { C.2 Effective action } & 59\end{array}$

$\begin{array}{lll}\text { C.3 Canonical quantization } & 60\end{array}$

$\begin{array}{lll}\text { C.3.1 Bosonic system } & 60\end{array}$

$\begin{array}{ll}\text { C.3.2 Supersymmetric system } & 61\end{array}$

$\begin{array}{ll}\text { D Quantization of Chern-Simons theory } & 64\end{array}$

D.1 Generalities 64

D.2 Boundary conditions $\quad 65$

D.3 Real polarization $\quad 66$

$\begin{array}{lll}\text { D.3.1 } \Sigma=D^{2} & 66\end{array}$

D.3.2 $\Sigma=T^{2} \quad 67$

$\begin{array}{lll}\text { D. } & \text { Holomorphic polarization } & 68\end{array}$

$\begin{array}{ll}\text { D.4.1 Coherent states } & 68\end{array}$

$\begin{array}{lll}\text { D.4.2 } \Sigma=T^{2} \text { redux } & 69\end{array}$

$\begin{array}{ll}\text { E Surgery versus localization } & 70\end{array}$

\section{Introduction}

The goal of this paper is to argue that certain properties of three-dimensional ChernSimons theory can be understood in a unified way by regarding the theory as an effective description of an $\mathcal{N}=2$ supersymmetric completion.

The application of supersymmetry to topological field theories is far from new. For instance, both the topological invariance and semiclassical exactness of observables in Wittentype (cohomological) TQFTs have long been recognized as consequences of a fermionic BRST symmetry [1]. After a suitable topological twist, gauge-fixed Chern-Simons theory itself furnishes an example of a Witten-type TQFT [2]. The BRST supersymmetry is a restatement of the underlying general covariance of the theory: the subtraction of ghost degrees of freedom guarantees the absence of excited states. By contrast, our approach relies on a further auxiliary supersymmetry. The relevant fermions obey the spin-statistics 
theorem. At finite Yang-Mills coupling, they result in an infinite tower of states with equal numbers of bosonic and fermionic degrees of freedom, which make no net contribution to supersymmetric observables. However, they have the additional effect of shifting the number of vacuum states. We will argue that this shift, combined with the localization principle afforded by the auxiliary fermionic symmetry, provides a natural framework in which to understand some features of correlation functions in bosonic Chern-Simons theory that are obscure from the point of view of perturbation theory.

\subsection{Old perspectives}

Let us first recall what is known. It has long been understood that induced Chern-Simons terms are one-loop exact because higher-order corrections (via an expansion in $\hbar \sim 1 / k$ ) cannot, in general, respect the quantization condition on the level [3, 4] (see [5] for a diagrammatic proof in the abelian case, and $[6,7]$ for a modern perspective). One manifestation of this fact is that quantum observables in pure Chern-Simons theory with simple gauge group $G$ and level $k>0$, possibly involving Wilson loops in irreducible representations of $G$ labeled by highest weights $\lambda$, are naturally viewed as functions not of the "bare" parameters (suitably defined), but of

$$
k \rightarrow k+h, \quad \lambda \rightarrow \lambda+\rho
$$

where $h$ is the dual Coxeter number and $\rho$ is the Weyl vector of $G$. For example, when $G=\mathrm{SU}(2)$, the shifts read $k \rightarrow k+2$ and $j \rightarrow j+1 / 2$, and the latter appears at the level of representation theory in the $\mathrm{SU}(2)$ Weyl character

$$
\chi_{j}(\theta)=\sum_{m=-j}^{j} e^{i m \theta}=\frac{\sin [(j+1 / 2) \theta]}{\sin (\theta / 2)},
$$

which (up to a $j$-independent prefactor) takes the form of a sum over $m= \pm(j+1 / 2)$, as familiar from equivariant localization formulas (see $[8,9]$ and references therein). These shifts can be thought of as quantum corrections.

By now, exact results for Chern-Simons theory have been obtained by various methods that give different ways of understanding the level shift: aside from surgery and 2D CFT [10], these include abelianization on circle bundles over Riemann surfaces [11, 12], nonabelian localization [13, 14], and supersymmetric localization [2, 15]. Of particular relevance to the last approach (such as when performing supersymmetric tests of nonsupersymmetric dualities $[16,17]$ ) is the fact that correlation functions in pure $\mathcal{N}=2$ and $\mathcal{N}=0$ Chern-Simons coincide up to a shift of the above form: in the $\mathcal{N}=2$ Chern-Simons action at level $k+h$, all superpartners of the gauge field are auxiliary, and performing the Gaussian path integral over these fields leads to an effective $\mathcal{N}=0$ Chern-Simons action at level $k$.

While $\lambda$, unlike $k$, does not appear in the bulk Lagrangian, the associated shift similarly lends itself to a Lagrangian point of view via an auxiliary system attached to the Wilson line, obtained by quantizing the coadjoint orbit of $\lambda$. In fact, as we will explain, the weights $\lambda$ in $\mathcal{N}=2$ Chern-Simons theory are subject to a "non-renormalization principle" similar 
to that of $k$, as can be seen by localizing the corresponding 1D $\mathcal{N}=2$ theories on Wilson lines. ${ }^{1}$ The essence of the $1 \mathrm{D}$ localization argument appears in the prototypical system of a massless charged particle on $S^{2}$ in the field of a magnetic monopole, which we refer to as the "monopole problem." In [19], it is shown using a hidden supersymmetry that the semiclassical approximation to the path integral for the monopole problem is exact: rather than taking the zero-mass limit, one can introduce a fermionic superpartner so that the contributions of all excited states to the partition function cancel regardless of the mass. The upshot is a derivation of the Weyl character formula for $\mathrm{SU}(2)$ from supersymmetric quantum mechanics. The same strategy of localizing an apparently purely bosonic theory has many modern incarnations: see, for example, [20]. Part of our discussion involves giving a slightly more modern formulation of the treatment of the monopole problem in [19], while embedding it into Chern-Simons theory.

\subsection{New perspectives}

Our goal is to explain how supersymmetric localization provides a structural understanding of the aforementioned exact results in the sense that the essential mechanism for both shifts, in the supersymmetric context, is identical in 3D and in $1 \mathrm{D}$.

While the renormalized parameters in (1.1) are one-loop exact, general observables in the $\mathcal{N}=0$ theory are not, reflecting the fact that Chern-Simons theory is conventionally formulated as a Schwarz-type rather than a Witten-type TQFT. The real power of supersymmetry lies in its ability to explain how the shifts (1.1) persist nonperturbatively in a wide class of observables. Enhancing both the 3D Chern-Simons action and the 1D coadjoint orbit action for Wilson loops with $\mathcal{N}=2$ supersymmetry gives one access to a localization argument that ensures that correlation functions depend only on the bare couplings appearing in the respective actions. This is a sort of non-renormalization principle. These two supersymmetrizations are not independent, as there exists a precise map between fields in the bulk and fields on the line. The supersymmetric, coupled 3D-1D path integral can be evaluated exactly, and after adjusting for parity anomalies ${ }^{2}$ from integrating out the auxiliary fermions (in 3D and in 1D), we immediately deduce the exact result in the corresponding bosonic theory, including the famous shifts. In this way, a one-loop supersymmetric localization computation reproduces an all-loop result in the bosonic theory. This reasoning leads to a conceptually simpler explanation for (1.1) than that originally obtained from anomalies in the coherent state functional integral [21].

Making the above statements precise requires fixing unambiguous physical definitions of the "bare" parameters $k$ and $\lambda$ - for example, via the coefficient of the two-point function in the associated $2 \mathrm{D}$ current algebra and canonical quantization of the coadjoint

\footnotetext{
${ }^{1} 3 \mathrm{D} \mathcal{N} \geq 2$ theories are precisely those whose holomorphy properties allow them to be constrained by non-renormalization theorems [18].

${ }^{2}$ We are abusing terminology here: by this, we simply mean the trading of a parity-violating fermion mass for a parity-violating Chern-Simons term. The induced Chern-Simons terms that we obtain from integrating out massive fermions will always be properly quantized, so we will not encounter any actual parity anomalies (the situation is different when $\mathcal{N}=1[4]$ ).
} 
orbit theory, respectively. ${ }^{3}$ Having done so, the shifts in $k$ and $\lambda$ arise in a unified fashion from jointly supersymmetrizing the 3D bulk theory and the $1 \mathrm{D}$ coadjoint orbit theory, giving rise to three equivalent descriptions of the same theory:

1. The bosonic Chern-Simons theory has level $k$ and Wilson loops

$$
\operatorname{Tr}_{\lambda} P \exp \left(i \oint A_{\mu} d x^{\mu}\right) \text {. }
$$

2. The supersymmetric Chern-Simons theory has level $k+h$ and Wilson loops

$$
\operatorname{Tr}_{\lambda} P \exp \left[i \oint\left(A_{\mu} d x^{\mu}-i \sigma d s\right)\right]
$$

where $\sigma$ is the real scalar in the vector multiplet.

3. The coadjoint orbit description of half-BPS Wilson loops coupled to the bulk supersymmetric theory has level $k+h$ and weight $\lambda+\rho$ from the start; these parameters are not renormalized. The trace in (1.4) is replaced by an appropriate supertrace in a 1D theory containing an auxiliary complex fermion $\psi$. In the standard presentation of a supersymmetric Wilson loop, the fermion $\psi$ has already been integrated out.

One would in principle expect to be able to match all observables between these descriptions, not only those that are protected (BPS) and hence calculable using supersymmetric localization, because the path integral over the auxiliary fields can be performed exactly (shifting $(k+h, \lambda+\rho) \rightarrow(k, \lambda)$ and setting $\sigma=0$, respectively). The main limitation of our analysis is that we are able to demonstrate this equivalence only for correlation functions of Wilson loops that are BPS with respect to the bulk supersymmetry (for which the integration contour implicit in (1.4) is subject to certain constraints).

Our approach involves introducing an auxiliary fermionic symmetry with the aid of generalized Killing spinors, allowing the localization procedure to be carried out on arbitrary Seifert manifolds. The underlying geometric structure that makes this possible is a transversely holomorphic foliation, or THF [24, 25]. It is worth contrasting this approach with that of [2], which avoids assuming the existence of Killing spinors by using a contact structure to define the requisite fermionic symmetry. A contact structure exists on any compact, orientable three-manifold. It is, locally, a one-form $\kappa$ for which $\kappa \wedge d \kappa \neq 0$; a metric can always be chosen for which $\kappa \wedge d \kappa$ is the corresponding volume form, i.e., such that $* 1=\kappa \wedge d \kappa$ and $* \kappa=d \kappa$. The dual vector field $v$ such that $\iota_{v} \kappa=1$ and $\iota_{v} d \kappa=0$ is known

\footnotetext{
${ }^{3}$ An intrinsically bulk definition of $k$ is as follows. For positive integer $k$, the Hilbert space of ChernSimons theory with simply connected $G$ on a Riemann surface $\Sigma$ is isomorphic to $H^{0}\left(\mathcal{M}, \mathcal{L}^{k}\right)$ where $\mathcal{M}$ is the moduli space of flat $G$-connections on $\Sigma$ and $\mathcal{L}$ is the basic line bundle over $\mathcal{M}$ in the sense of having positive curvature and that all other line bundles over $\mathcal{M}$ take the form $\mathcal{L}^{n}$ for some integer $n$ [4]. For example, for simple, connected, simply connected $G$ and $\Sigma=T^{2}, \mathcal{M}$ is a weighted projective space of complex dimension $\operatorname{rank} G$ and $\mathcal{L}=\mathcal{O}(1)$ (whose sections are functions of degree one in homogeneous coordinates on $\mathcal{M}$ ). In the $\mathcal{N}=1$ and $\mathcal{N}=2$ settings, fermions have the effect of tensoring $\mathcal{L}^{k}$ with $K^{1 / 2}$ or $K$ to give $\mathcal{L}^{k-h / 2}$ or $\mathcal{L}^{k-h}$, respectively, where $K=\mathcal{L}^{-h}$ is the canonical bundle of $\mathcal{M}$. Note that these fermions effectively implement the metaplectic correction in geometric quantization [22, 23].
} 
as the Reeb vector field. It was found in [2] that to carry out the localization, the corresponding Reeb vector field must be a Killing vector field, which restricts this approach to Seifert manifolds (as in [14]); this approach was generalized in [26] to Chern-Simons theories with matter. Therefore, while the geometric basis for our approach differs from that for the cohomological localization of $[2,26]$, the domain of applicability is the same. Our focus, however, is different: the compensating level shift from auxiliary fermions was ignored in [2], noted in [26], and essential in neither.

We begin by reviewing some background material and setting our conventions in sections 2 and 3. We then carry out the analysis for Wilson lines very explicitly for $G=\mathrm{SU}(2)$ in section 4 (we comment briefly on the generalization to arbitrary $G$ at the end of the paper). Using the description of these lines as $1 \mathrm{D} \mathcal{N}=2$ sigma models, we compute the effective action for fermions at both zero and finite temperature, canonically quantize the system, and present the localization argument in 1D.

In section 5 , we show how to embed this story in bulk $3 \mathrm{D} \mathcal{N}=2$ Chern-Simons theory. Crucially, while we expect $\mathcal{N}=0$ and $\mathcal{N}=2$ Chern-Simons to be equivalent by integrating out the extra fields in the vector multiplet, the equivalence only holds if we take into account both the shift of the level and the weight (as discussed further in section 7).

In section 6, we describe how to generalize the aforementioned analysis of a Wilson line in flat space, either straight or wrapping a compact direction, to various classes of compact three-manifolds. We also give some examples of the observables that we can compute. Both the $\mathcal{N}=0$ and $\mathcal{N}=2$ theories are topological, so their observables are metric-independent. In the $\mathcal{N}=0$ case, the introduction of a metric is usually regarded as a "necessary evil" for the purposes of gauge-fixing and regularization. In the $\mathcal{N}=2$ case, the metric plays a more essential role in computing observables because it determines which observables are compatible with supersymmetry and therefore accessible to localization techniques. Seifert loops (i.e., Wilson loops along the Seifert fiber direction) can give different knots depending on the choice of Seifert fibration. For instance, depending on the choice of Seifert fibration on $S^{3}$, the half-BPS sector can contain Wilson loop configurations with the topology of Hopf links or torus links [14].

We review in appendix D the necessary elements of the quantization of Chern-Simons theory to which we refer throughout the paper. In appendix E, we comment on SUSY as an alternative to surgery computations in some situations.

\section{$2 \mathcal{N}=0$ Chern-Simons theory}

Let $M^{3}$ be a compact, oriented three-manifold and let $G$ be a simple, compact, connected, simply connected Lie group. The latter two assumptions on $G$ ensure that any principal $G$-bundle $P$ over $M^{3}$ is trivial, so that the Chern-Simons gauge field $A$ is a connection on all of $P$. It then suffices to define the Lorentzian $\mathcal{N}=0 G_{k>0}$ Chern-Simons action by

$$
S_{\mathrm{CS}}=\frac{k}{4 \pi} \int_{M^{3}} \operatorname{Tr}\left(A d A-\frac{2 i}{3} A^{3}\right) .
$$

We normalize the trace such that the norm squared of the longest root is two (for example, when $G=\mathrm{SU}(N)$, the trace is taken in the fundamental representation and $k$ is integrally 
quantized). In more general settings (e.g., $G$ non-simply connected), the quantization of $k$ would depend on additional data, such as whether we choose a spin structure on $M^{3}$ [27].

In flat space, we work in Lorentzian signature, except when computing the supersymmetric index in section 4.3. In curved space (section 6), we work in Euclidean signature. In flat Minkowski space, we have the $\mathcal{N}=2$ Lagrangians

$$
\begin{aligned}
\left.\mathcal{L}_{\mathrm{CS}}\right|_{\mathbb{R}^{1,2}} & =\frac{k}{4 \pi} \operatorname{Tr}\left[\epsilon^{\mu \nu \rho}\left(A_{\mu} \partial_{\nu} A_{\rho}-\frac{2 i}{3} A_{\mu} A_{\nu} A_{\rho}\right)-2 i \lambda \bar{\lambda}-2 D \sigma\right], \\
\left.\mathcal{L}_{\mathrm{YM}}\right|_{\mathbb{R}^{1,2}} & =\frac{1}{g^{2}} \operatorname{Tr}\left(-\frac{1}{4} F_{\mu \nu} F^{\mu \nu}-\frac{1}{2} D_{\mu} \sigma D^{\mu} \sigma+\frac{1}{2} D^{2}+i \bar{\lambda} \gamma^{\mu} D_{\mu} \lambda-i \bar{\lambda}[\sigma, \lambda]\right) .
\end{aligned}
$$

These are written in the convention where the generators $T^{a}$ are Hermitian, which we use throughout this paper. ${ }^{4}$

\subsection{Perturbation theory}

The level of the pure $\mathcal{N}=2$ CS theory whose correlation functions reproduce those of the corresponding $\mathcal{N}=0$ theory is $k_{\mathcal{N}=2}=k_{\mathcal{N}=0}+h\left(k_{\mathcal{N}=0}>0\right.$ by assumption). This we refer to as the "fermionic shift": the IR effective action $S_{\text {eff }}[A, m]$ for two adjoint Majorana fermions with real mass $m$, minimally coupled to a $G$-gauge field, is $S_{\mathrm{CS}}$ at level $h \operatorname{sign}(m)[29,30]$. Specifically, consider the sum of (2.2) and (2.3). The resulting theory has a mass gap of $m=k g^{2} / 2 \pi$. At large $k\left(m \gg g^{2}\right)$, we may integrate out all massive superpartners of the gauge field. Assuming unbroken supersymmetry, the result is the lowenergy effective theory of zero-energy supersymmetric ground states. Of course, the fact that integrating out $\lambda$ induces $\mathcal{L}_{\mathrm{CS}}^{\mathcal{N}}=0$ at level $-h$ (among other interactions), along with the assumption that $\mathcal{N}=2$ SUSY is preserved quantum-mechanically, is only a heuristic justification for the renormalization of the coefficient of $\mathcal{L}_{\mathrm{CS}}^{\mathcal{N}=2}$ to $k-h$. This expectation is borne out by computing the one-loop perturbative renormalization of couplings [28].

The fermionic shift discussed above is entirely separate from any "bosonic shift" that might arise from gauge dynamics (as found in, e.g., [31], which effectively integrates out the topologically massive $W$-boson). Such a shift does not affect the number of vacuum states. Indeed, it is an artifact of regularization scheme: in the YM-CS regularization (which preserves supersymmetry, and which we use throughout this paper), the IR level is shifted by $+h$ relative to the bare level, while dimensional regularization yields no such shift [3]. It is, nonetheless, a convenient conceptual slogan that $k$ is renormalized to $k+h$ at one loop in $\mathcal{N}=0$ YM-CS, so that $k$ is not renormalized in $\mathcal{N} \geq 2$ YM-CS. The important point is that for $\mathcal{N} \geq 2$ supersymmetry, integrating out the gauginos in the 3D YM-CS Lagrangian yields a shift of $-h$, which is twice the shift of $-h / 2$ in the $\mathcal{N}=1$ case [28].

Given a precise physical definition of the level $k$, such as those presented in the introduction, a more substantive "bosonic" shift of the form mentioned above is that exhibited by correlation functions of $\mathcal{N}=0$ Chern-Simons theory as functions of $k$. This can already

\footnotetext{
${ }^{4}$ Writing $\lambda=(\eta+i \tilde{\eta}) / \sqrt{2}$ with $\eta, \tilde{\eta}$ real adjoint Majorana fermions reproduces the $\mathcal{N}=2$ expressions of [28]. WLOG, we may take $k>0$ because time reversal (equivalently, spacetime orientation reversal in Euclidean signature) flips the overall sign of (2.2), i.e., the sign of the bosonic Chern-Simons term, the sign of the gaugino mass term, and the sign of the pseudoscalar $\sigma$.
} 
be seen in the semiclassical limit [10]. At large $k$, we may expand (2.1) to quadratic order around a flat connection $A_{0}$. The semiclassical path integral evaluates to its classical value weighted by the one-loop contribution $e^{i \pi \eta\left(A_{0}\right) / 2} T\left(A_{0}\right)$ where $T\left(A_{0}\right)$ is the Ray-Singer torsion of $A_{0}$. The APS index theorem implies that the relative $\eta$-invariant

$$
\frac{1}{2}\left(\eta\left(A_{0}\right)-\eta(0)\right)=\frac{h}{\pi} I\left(A_{0}\right)
$$

where $I\left(A_{0}\right) \equiv \frac{1}{k} S_{\mathrm{CS}}\left(A_{0}\right)$, is a topological invariant. The large- $k$ partition function is then

$$
Z=e^{i \pi \eta(0) / 2} \sum_{\alpha} e^{i(k+h) I\left(A_{0}^{(\alpha)}\right)} T\left(A_{0}^{(\alpha)}\right),
$$

where the sum (assumed finite) runs over gauge equivalence classes of flat connections. This is how the shift $k \rightarrow k+h$, which persists in the full quantum answer, appears perturbatively. The phase $\eta(0)$ depends on the choice of metric. However, given a trivialization of the tangent bundle of $M^{3}$, the gravitational Chern-Simons action $I_{\text {grav }}(g)$ has an unambiguous definition, and upon adding a counterterm $\frac{\operatorname{dim} G}{24} I_{\text {grav }}(g)$ to the action, the resulting large- $k$ partition function is a topological invariant of the framed, oriented three-manifold $M^{3}[10]$.

Thus a framing of $M^{3}$ fixes the phase of $Z$. Aside from the framing anomaly of $M^{3}$ itself, there exists a framing ambiguity of links within it. This point will be important in our application: the supersymmetric framing of a BPS Wilson loop differs from the canonical framing, when it exists, because the point splitting that determines the self-linking number must be performed with respect to another BPS loop [15].

To make concrete the utility of supersymmetry in light of these perturbative considerations, take as an example $\mathcal{N}=0 \mathrm{SU}(2)_{k}$ on $S^{3}$. A typical observable in this theory receives contributions from all loops. For example, the partition function is

$$
Z\left(S^{3}\right)=\sqrt{\frac{2}{k+2}} \sin \left(\frac{\pi}{k+2}\right) .
$$

Suppose we were to compute the logarithm of this quantity (the free energy on $S^{3}$ ) in perturbation theory as the sum of connected vacuum bubbles, without recourse to $2 \mathrm{D}$ conformal field theory. Expanding around the trivial flat connection, the one-loop factor is simply the large- $k$ limit of the exact result:

$$
Z_{\text {1-loop }}=\exp (\bigcirc)=\frac{\sqrt{2} \pi}{(k+2)^{3 / 2}}
$$

The reconstruction of the exact result from summing trivalent graphs is far from obvious, regardless of whether the expansion parameter is $k^{-1}$ or $(k+2)^{-1}$ (the necessity of doing perturbation theory in the renormalized level has historically been a point of contention in the literature; for a review of early references on large- $k$ asymptotics of Chern-Simons invariants, see [32]). On the other hand, a one-loop supersymmetric localization computation in $\mathcal{N}=2 \mathrm{SU}(2)_{k+2}$ on $S^{3}$ (with the level adjusted to account for the fermionic shift, suitably generalized to curved space) handily yields the all-loop non-supersymmetric result (2.6), up to a framing phase given in appendix E. The bulk of our discussion will focus on more complicated observables that include Wilson loops. 


\subsection{Beyond perturbation theory}

As known since [10], there exist completely general nonperturbative techniques for computing observables in the $\mathcal{N}=0$ theory, and thus checks of any results obtained via supersymmetry. These techniques rely on essentially two ingredients. The first is the fact that $Z\left(\Sigma \times_{K} S^{1}\right)=\operatorname{Tr}_{\mathcal{H}_{\Sigma}}(K)$, where the mapping torus $\Sigma \times_{K} S^{1}$ is obtained by identifying the ends of the cylinder $\Sigma \times[0,1]$ by a diffeomorphism $K$ of $\Sigma$. The second is the fundamental surgery formula

$$
Z\left(\tilde{M} ; R_{i}\right)=\sum_{j} K_{i}^{j} Z\left(M ; R_{j}\right),
$$

where $M$ contains an arbitrary Wilson loop in the representation $R_{i}$ (possibly trivial) and $\tilde{M}$ is the result of gluing a tubular neighborhood of this loop back into $M$ with a diffeomorphism $K$ on its boundary. Topologically equivalent surgeries on three-manifolds may have different effects on framing.

To give a few examples of nonperturbative results computed by these means (stated in the canonical framing), consider $G_{k>0}$ on $S^{3}$. Let $S_{i j}$ be the representation of the modular transformation $S$ on $T^{2}$ in the Verlinde basis for $\mathcal{H}_{T^{2}}$. Then

$$
Z\left(S^{3}\right)=S_{00}=\frac{1}{(k+h)^{\operatorname{rank} G / 2}}\left(\frac{\operatorname{vol} \Lambda_{W}}{\operatorname{vol} \Lambda_{R}}\right)^{1 / 2} \prod_{\alpha>0} 2 \sin \left(\frac{\pi \alpha(\rho)}{k+h}\right),
$$

while for an unknotted Wilson loop in an irreducible representation $R_{i}$,

$$
\langle W\rangle=\frac{Z\left(S^{3} ; R_{i}\right)}{Z\left(S^{3}\right)}=\frac{S_{0 i}}{S_{00}}=\prod_{\alpha>0} \frac{\sin (\pi \alpha(\lambda+\rho) /(k+h))}{\sin (\pi \alpha(\rho) /(k+h))} .
$$

Here, $\alpha$ runs over positive roots and $\lambda$ is the highest weight of $R_{i}$. The expressions in terms of $S$-matrix elements were deduced in [10], while the explicit formulas in (2.9) and (2.10) are consequences of the Weyl denominator and character formulas [33]. ${ }^{5}$ In particular, for $\mathrm{SU}(2)_{k}$,

$$
S_{i j}=\sqrt{\frac{2}{k+2}} \sin \left[\frac{(2 i+1)(2 j+1) \pi}{k+2}\right]
$$

where $i, j$ label the spins of the corresponding representations (thus giving (2.6)), and for an unknotted Wilson loop in the spin- $j$ representation,

$$
\langle W\rangle=\frac{S_{0 j}}{S_{00}}=\frac{q^{j+1 / 2}-q^{-(j+1 / 2)}}{q^{1 / 2}-q^{-1 / 2}}=\frac{\sin ((2 j+1) \pi /(k+2))}{\sin (\pi /(k+2))}
$$

where $q=e^{2 \pi i /(k+2)}$.

In some observables, highest weights of integrable representations of the $G_{k}$ theory appear not due to explicit Wilson loop insertions, but rather because they are summed

\footnotetext{
${ }^{5}$ The result for $Z\left(S^{3}\right)$ follows from consistency between two different ways of gluing together two copies of a solid torus $D^{2} \times S^{1}$ : one trivially to get $S^{2} \times S^{1}$, and another with an $S$ transformation on the boundary to get $S^{3}$. More generally, by inserting Wilson lines in these solid tori, one obtains the expectation value of the Hopf link as a normalized $S$-matrix element.
} 
over. Indeed, the shift in $\lambda$ already appears in the partition function on $\Sigma \times S^{1}$, which computes the dimension of the Hilbert space of the Chern-Simons theory on $\Sigma$ and hence the number of conformal blocks in the corresponding 2D RCFT. The answer is famously given by the Verlinde formula, which for arbitrary compact $G$, reads [11]

$$
\operatorname{dim} V_{g, k}=\left(|Z(G)|(k+h)^{\operatorname{rank} G}\right)^{g-1} \sum_{\lambda \in \Lambda_{k}} \prod_{\alpha}\left(1-e^{2 \pi i \alpha(\lambda+\rho) /(k+h)}\right)^{1-g}
$$

where $g$ is the genus of $\Sigma$ and $\Lambda_{k}$ denotes the set of integrable highest weights of $\widehat{G}_{k}$. While our focus is on Wilson loops, it turns out that the appearance of $\lambda+\rho$ in $Z\left(\Sigma \times S^{1}\right)$ comes "for free" in our approach, without the need to adjust for any 1D fermionic shifts, which is consistent with the fact that the weights in (2.13) are not associated with Wilson loops. This fact has already been appreciated in prior literature. ${ }^{6}$

\section{$3 \quad$ Wilson loops and coadjoint orbits}

\subsection{The orbit method}

A central ingredient in our analysis is the fact that a Wilson loop over a curve $\gamma$ in $M^{3}$ is a path integral for a $1 \mathrm{D}$ Chern-Simons theory whose classical phase space is a coadjoint orbit of $G$, with the corresponding representation $R$ arising by the orbit method [10]. We will be interested in the case of compact $G$, where this construction is also known as BorelWeil-Bott quantization. The philosophy is that one can eliminate both the trace and the path ordering from the definition of a Wilson loop in a nonabelian gauge theory at the cost of an additional path integral over all gauge transformations along $\gamma$.

To make this description explicit, we draw from the exposition of [14]. We would like to interpret a Wilson loop as the partition function of a quantum-mechanical system on $\gamma$ with time-dependent Hamiltonian. In the Hamiltonian formalism, this is a matter of writing

$$
W_{R}(\gamma)=\operatorname{Tr}_{R} P \exp \left(i \oint_{\gamma} A\right)=\operatorname{Tr}_{\mathcal{H}} T \exp \left(-i \oint_{\gamma} H\right)
$$

where the Hilbert space $\mathcal{H}$ is the carrier space of the representation $R, H$ generates translations along $\gamma$, and the time evolution operator is the holonomy of the gauge field. In the path integral formalism, this becomes

$$
W_{R}(\gamma)=\int D U e^{i S_{\lambda}\left(U,\left.A\right|_{\gamma}\right)}
$$

where $U$ is an auxiliary bosonic field on $\gamma, \lambda$ is the highest weight of $R$, and the restriction of the bulk gauge field $\left.A\right|_{\gamma}$ is a background field in the (operator-valued) path integral over $U$. Since the definition of a Wilson loop is independent of any metric on $\gamma,{ }^{7}$ it is not surprising that the action $S_{\lambda}$ will turn out to describe a topological sigma model.

\footnotetext{
${ }^{6}$ Supersymmetric localization has been used to compute the Verlinde formula in genus zero [34] and in arbitrary genus [35, 36], reproducing the result of [11] for $Z_{T^{3}}=\operatorname{dim} \mathcal{H}_{T^{2}}$ when $g=1$.

${ }^{7}$ This is not true of its supersymmetric counterparts.
} 
The Borel-Weil-Bott theorem identifies the irreducible representation $R$ with the space of holomorphic sections of a certain line bundle over the coadjoint orbit $\mathcal{O}_{\lambda} \subset \mathfrak{g}^{*}$ of $\lambda$, which (in the generic case) is isomorphic to the flag manifold $G / T$ where $T$ is a maximal torus of $G$. In physical terms, it states that $R$ is the Hilbert space obtained by quantizing $\mathcal{O}_{\lambda}$. We are therefore led to consider the quantum mechanics of a particle on $\mathcal{O}_{\lambda}$ given by a $1 \mathrm{D}$ sigma model of maps $U: S^{1} \rightarrow \mathcal{O}_{\lambda}$, where the compact worldline is identified with $\gamma \subset M^{3}$. To ensure that $\mathcal{O}_{\lambda}$ (rather than $T^{*} \mathcal{O}_{\lambda}$ ) appears as the classical phase space, the action for $U$ must be first-order in the time derivative along $S^{1}$. Moreover, on general grounds, it should be independent of the metric on $S^{1}$.

There is an essentially unique choice of action that fulfills these wishes. For convenience, we identify $\lambda$ via the Killing form as an element of $\mathfrak{g}$ rather than $\mathfrak{g}^{*}$, so that $\mathcal{O}_{\lambda} \subset \mathfrak{g}$ is the corresponding adjoint orbit (henceforth, we shall not be careful to distinguish $\mathfrak{g}$ and $\left.\mathfrak{g}^{*}\right)$. We assume that $\lambda$ is a regular weight, so that $\mathcal{O}_{\lambda} \cong G / G_{\lambda}$ where $G_{\lambda} \cong T$. The (left-invariant) Maurer-Cartan form $\theta$ is a distinguished $\mathfrak{g}$-valued one-form on $G$ that satisfies $d \theta+\theta \wedge \theta=0$. We obtain from it two natural forms on $G$, namely the real-valued presymplectic one-form $\Theta_{\lambda}$ and the coadjoint symplectic two-form $\nu_{\lambda}$ :

$$
\theta=g^{-1} d g \in \Omega^{1}(G) \otimes \mathfrak{g}, \quad \Theta_{\lambda}=i \operatorname{Tr}(\lambda \theta) \in \Omega^{1}(G), \quad \nu_{\lambda}=d \Theta_{\lambda} \in \Omega^{2}(G) .
$$

Both $\Theta_{\lambda}$ and $\nu_{\lambda}$ descend to forms on $\mathcal{O}_{\lambda}$. The weight $\lambda$ naturally determines a splitting of the roots of $G$ into positive and negative, positive roots being those having positive inner product with $\lambda$. Endowing $\mathcal{O}_{\lambda}$ with the complex structure induced by this splitting makes $\mathcal{O}_{\lambda}$ a Kähler manifold, with Kähler form $\nu_{\lambda}$ of type $(1,1) .{ }^{8}$ Now consider the action

$$
S_{\lambda}(U)=\oint_{S^{1}} U^{*}\left(\Theta_{\lambda}\right)=\oint_{S^{1}}\left(\Theta_{\lambda}\right)_{m} \frac{d U^{m}}{d \tau} d \tau
$$

The second expression (written in local coordinates $U^{m}$ on $\mathcal{O}_{\lambda}$ ) is indeed first-order in derivatives, so that the solutions to the classical EOMs are constant maps $U$, as desired.

To be concrete, we may think of $U$ as parametrizing gauge transformations. Using the isomorphism $G / G_{\lambda} \stackrel{\sim}{\rightarrow} \mathcal{O}_{\lambda}$ given by $g G_{\lambda} \mapsto g \lambda g^{-1}$, we lift $U$ to a map $g: S^{1} \rightarrow G$, so that

$$
S_{\lambda}(U)=i \oint_{S^{1}} \operatorname{Tr}\left(\lambda g^{-1} d g\right)
$$

From (3.5), we see very explicitly that the canonical symplectic form $\nu_{\lambda}$ on $\mathcal{O}_{\lambda}$, given in (3.3), takes the form $d \pi_{g} \wedge d g$ where the components of $g$ are canonical coordinates. The fact that $\lambda \in \mathfrak{g}$ is quantized as a weight of $G$ implies that (3.5) is independent of the choice of lift from $\mathcal{O}_{\lambda}$ to $G$. Namely, $g$ is only determined by $U$ up to the right action of $G_{\lambda}$; under a large gauge transformation $g \mapsto g h$ where $h: S^{1} \rightarrow G_{\lambda}$, the integrand of (3.5)

\footnotetext{
${ }^{8}$ This is usually phrased as a choice of Borel subalgebra $\mathfrak{b} \supset \mathfrak{t}$, so that the coadjoint orbit is isomorphic to $G_{\mathbb{C}} / B$ where $B$ is the corresponding Borel subgroup and the roots of $B$ are defined to be the positive roots of $G$; then representations are labeled by their lowest weights. We instead adhere to the "highest weight" conventions of [14].
} 
changes by $d \operatorname{Tr}(\lambda \log h)$ and the action changes by an integer multiple of $2 \pi .^{9}$ Thus $\Theta_{\lambda}$ descends (up to exact form) to $\mathcal{O}_{\lambda}$. The path integral (3.2) is over all maps $U$ in $L \mathcal{O}_{\lambda}$, or equivalently, over all maps $g$ in $L G / L G_{\lambda}$ (accounting for the gauge redundancy).

To couple (3.5) to the bulk gauge field, we simply promote $d g$ to $d_{A} g=d g-\left.i A\right|_{\gamma} \cdot g$ :

$$
S_{\lambda}\left(U,\left.A\right|_{\gamma}\right)=i \oint_{S^{1}} \operatorname{Tr}\left(\lambda g^{-1} d_{A} g\right)
$$

Prescribing the correct gauge transformations under $G \times T$ (with $T$ acting on the right and $G$ acting on the left), the 1D Lagrangian transforms by the same total derivative as before.

The first-order action (3.5), in the absence of a background gauge field, can be thought of as describing the IR limit of a charged particle on $\mathcal{O}_{\lambda}$ in a magnetic field $\nu_{\lambda}$. In complete analogy to 3D Chern-Simons theory, the irrelevant two-derivative kinetic terms have the effect of renormalizing $\lambda$ to $\lambda+\rho$ at one loop, and upon supersymmetrizing the theory, the fermion effective action provides a compensating shift by $-\rho .{ }^{10}$ We will substantiate this interpretation for $G=\mathrm{SU}(2)$ in exhaustive detail.

\subsection{Wilson/'t Hooft loops in Chern-Simons theory}

While the coadjoint representation of a Wilson loop holds in any gauge theory, it is especially transparent in Chern-Simons theory, where it can be derived straightforwardly via a surgery argument [37]. Consider Chern-Simons on $S^{1} \times \mathbb{R}^{2}$, where the Wilson line wraps the $S^{1}$ at a point on the $\mathbb{R}^{2}$. Cutting out a small tube around $\gamma$ and performing a gauge transformation $\tilde{g}$, the action changes by ${ }^{11}$

$$
\Delta S=-\frac{i k}{2 \pi} \int_{\partial M^{3}} \operatorname{Tr}\left(A \tilde{g}^{-1} d \tilde{g}\right) .
$$

Set $\tilde{g}=e^{i \alpha \phi}$ where $e^{2 \pi i \alpha}=1$ (this gauge transformation is singular along the loop; $t$ is the coordinate along $\gamma$ and $\phi$ the coordinate around it). To define a gauge-invariant operator, average over $\tilde{g} \rightarrow g \tilde{g}$ and $A \rightarrow g A g^{-1}-i d g g^{-1}$ where $g=g(t)$, whereupon this becomes

$$
\Delta S=i k \int_{\gamma} \operatorname{Tr}\left(\alpha g\left(\partial_{t}-i A_{t}\right) g^{-1}\right) d t
$$

where we have performed the $\phi$ integral and shrunk the boundary to a point. Finally, replace $g$ by $g^{-1}$. Hence $k \alpha$ must be quantized as a weight $\lambda .{ }^{12}$ This derivation illustrates that Wilson and 't Hooft/vortex [38-40] loops are equivalent in pure Chern-Simons theory.

\footnotetext{
${ }^{9}$ From the geometric quantization point of view, the quantization of $\lambda$ is necessary for the existence of a prequantum line bundle $\mathcal{L}(\lambda)$ over $\mathcal{O}_{\lambda}$, with curvature $\nu_{\lambda}$. Each $\lambda$ in the weight lattice gives a homomorphism $\rho_{\lambda}: T \rightarrow \mathrm{U}(1)$, which can be used to construct an associated line bundle $\mathcal{L}(\lambda)=G \times_{\rho_{\lambda}} \mathbb{C}$ over $G / T$, so that the Hilbert space is the space of holomorphic sections of $\mathcal{L}(\lambda)$. Then $\Theta_{\lambda}$ is a connection on $\mathcal{L}(\lambda)$.

${ }^{10} \mathrm{As}$ in $3 \mathrm{D}$, the effect of these fermions can be compared to that of the metaplectic correction in geometric quantization, which states that wavefunctions should not be viewed as sections of $\mathcal{L}(\lambda)$, but rather as halfdensities valued in $\mathcal{L}(\lambda)$, meaning that they belong to $\mathcal{L}(\lambda) \otimes K^{1 / 2} \cong \mathcal{L}(\lambda-\rho)$ where $K^{1 / 2}$ is a square root of the canonical bundle of $\mathcal{O}_{\lambda}[22]$.

${ }^{11}$ By appendix A.1, varying the bulk action gives a boundary term of $-\frac{i k}{4 \pi} d\left(A \tilde{g}^{-1} d \tilde{g}\right)$; the Pontryagin density term does not contribute because $G$ is assumed simply connected. By appendix D.2, specifying nonzero $A_{t}$ on the boundary requires adding a boundary term of $\frac{k}{4 \pi} \int_{\partial M^{3}} d^{2} x \operatorname{Tr}\left(A_{t} A_{\phi}\right)$ to the action, whose variation under a $\phi$-dependent gauge transformation $\tilde{g}$ gives another contribution of $-\frac{i k}{4 \pi} \int_{\partial M^{3}} d^{2} x \operatorname{Tr}\left(A_{t} \tilde{g}^{-1} \partial_{\phi} \tilde{g}\right)$.

${ }^{12}$ We have corrected the transformation rule $\tilde{g} \rightarrow g \tilde{g} g^{-1}$ and a spurious factor of $\frac{1}{2}$ in [37].
} 
To summarize, consider a bulk theory with gauge group $G$ and the 1D Lagrangian

$$
\mathcal{L}_{1 \mathrm{D}}=i \operatorname{Tr}\left[\lambda g^{-1}\left(\partial_{t}-i A\right) g\right]
$$

where $g \in G,\left.A \equiv A\right|_{\gamma}$, and $\lambda \in \mathfrak{t}$ (properly, $\lambda \in \mathfrak{t}^{*}$ ). Since $\lambda$ is Hermitian in our conventions, the factor of $i$ ensures that the coadjoint orbit action is real. The Lagrangian (3.9) transforms by a total derivative under $t$-dependent $G \times T$ gauge transformations

$$
g \rightarrow h_{\ell} g h_{r}, \quad A \rightarrow h_{\ell} A h_{\ell}^{-1}-i \partial_{t} h_{\ell} h_{\ell}^{-1},
$$

namely $i \operatorname{Tr}\left(\lambda \partial_{t} \log h_{r}\right)$, where $h_{\ell}$ is the restriction of a $G$-gauge transformation in the bulk and $h_{r} \in T$. Hence $\lambda$ is quantized to be a weight of $G$. The $T$-gauge symmetry restricts the degrees of freedom in $g$ to $G / T$. Quantizing $g$ in this Lagrangian leads to the Wilson line.

Strictly speaking, the global symmetry of the model (3.5) that we gauge to obtain (3.9) is $G / Z(G)$, since the center is already gauged. This should be contrasted with the global symmetry $G \times G / Z(G)$ of a particle on a group manifold with the usual kinetic term $\operatorname{Tr}\left(\left(g^{-1} \dot{g}\right)^{2}\right)$, which consists of isometries of the bi-invariant Killing metric on $G$.

\section{Wilson loops in $\mathcal{N}=2$ Chern-Simons theory}

We now show that properly defining half-BPS Wilson loops in $\mathcal{N}=2$ Chern-Simons theory ensures that their weights are not renormalized, in direct parallel to the non-renormalization of the bulk Chern-Simons level. This involves enhancing the sigma model of the previous section with $1 \mathrm{D} \mathcal{N}=2$ supersymmetry in a way compatible with bulk $3 \mathrm{D} \mathcal{N}=2$ supersymmetry.

\subsection{Shift from line dynamics}

\subsection{1 $\mathcal{N}=2$ coadjoint orbit}

We work in Lorentzian $1 \mathrm{D} \mathcal{N}=2$ superspace with coordinates $\left(t, \theta, \theta^{\dagger}\right)$ (see appendix A.2). Implicitly, we imagine a quantum-mechanical system on a line embedded in $\mathbb{R}^{1,2}$, but we will not need to pass to 3D until the next section. Our primary case study is $G=\mathrm{SU}(2)$. We first construct, without reference to the 3D bulk, an $\mathrm{SU}(2)$-invariant and supersymmetric coadjoint orbit Lagrangian from the $1 \mathrm{D} \mathcal{N}=2$ chiral superfield

$$
\Phi=\phi+\theta \psi-i \theta \theta^{\dagger} \dot{\phi}
$$

descending from bulk super gauge transformations and the $1 \mathrm{D} \mathcal{N}=2$ vector superfields

$$
V_{i}=a_{i}+\theta \psi_{i}-\theta^{\dagger} \psi_{i}^{\dagger}+\theta \theta^{\dagger} A_{i}
$$

obtained from restrictions of the bulk fields to the Wilson line, which extends along the 0 direction in flat space. Here, $i=1,2,3$ label the $\mathfrak{s u}(2)$ components in the $\vec{\sigma} / 2$ basis; $\phi$ is a complex scalar and $\psi$ is a complex fermion; $a_{i}, A_{i}$ are real scalars and $\psi_{i}$ are complex fermions; and the relevant SUSY transformations are given in (A.10) and (A.12). 
We begin by writing (3.9) in a form more amenable to supersymmetrization, namely in terms of a complex scalar $\phi$ that parametrizes the phase space $\mathrm{SU}(2) / \mathrm{U}(1) \cong \mathbb{C P}^{1}$. Take $\lambda=-j \sigma_{3}$ with $j \in \frac{1}{2} \mathbb{Z}_{\geq 0}$, which fixes a Cartan; then

$$
g=\left(\begin{array}{cc}
a & b \\
-\bar{b} & \bar{a}
\end{array}\right), \quad|a|^{2}+|b|^{2}=1
$$

is subject to a $\mathrm{U}(1)$ gauge redundancy $g \sim g e^{i \theta \sigma_{3}}$. We identify variables via the Hopf map $\mathrm{SU}(2) \rightarrow S^{2}$, followed by stereographic projection:

$$
\phi=-\frac{a}{\bar{b}} \text {. }
$$

This map respects the chosen $\mathrm{U}(1)$ gauge equivalence: $(a, b) \rightarrow\left(a e^{i \theta}, b e^{-i \theta}\right)$. Let us gaugefix the $\mathrm{U}(1)$ action on the right by taking $b=r$ real. Since $|a|^{2}+r^{2}=1, r$ is only determined by $a$ up to a sign (reflecting the ambiguity in the action of $\mathrm{SU}(2)$ on $S^{2}$ ). Note that the gauge fixing breaks down when $|a|=1(r=0)$. Accounting for the sign ambiguity, we have

$$
\phi=-\frac{a}{ \pm \sqrt{1-|a|^{2}}} \Longrightarrow a=\mp \frac{\phi}{\sqrt{1+|\phi|^{2}}}, r= \pm \frac{1}{\sqrt{1+|\phi|^{2}}} .
$$

The relative minus sign is important for ensuring equivariance of the map from $a$ to $\phi$ with respect to the action of $\mathrm{SU}(2)$. Let us fix the overall sign to " $(a, r)=(+,-)$." This is a one-to-one map between the interior of the unit disk $|a|<1$ and the $\phi$-plane that takes the boundary of the disk to the point at infinity. To couple the $\phi$ degrees of freedom to the gauge field, we work in the basis $\vec{\sigma} / 2$, so that

$$
A=\frac{1}{2}\left(\begin{array}{cc}
A_{3} & A_{1}-i A_{2} \\
A_{1}+i A_{2} & -A_{3}
\end{array}\right)
$$

where the three $\mathfrak{s u}(2)$ components $A_{1,2,3}$ are real. Then the non-supersymmetric $1 \mathrm{D}$ coadjoint orbit Lagrangian (3.9) can be written as $\mathcal{L}_{1 \mathrm{D}}=j \mathcal{L}$ where $\mathcal{L}=\mathcal{L}_{0}+\mathcal{L}_{A}$ and

$$
\begin{aligned}
\mathcal{L}_{0} & =\frac{i}{j} \operatorname{Tr}\left(\lambda g^{-1} \partial_{t} g\right)=\frac{i\left(\phi \dot{\phi}^{\dagger}-\phi^{\dagger} \dot{\phi}\right)}{1+|\phi|^{2}}, \\
\mathcal{L}_{A} & =\frac{1}{j} \operatorname{Tr}\left(\lambda g^{-1} A g\right)=-\left[\frac{\left(A_{1}+i A_{2}\right) \phi+\left(A_{1}-i A_{2}\right) \phi^{\dagger}-A_{3}\left(1-|\phi|^{2}\right)}{1+|\phi|^{2}}\right] .
\end{aligned}
$$

Note that with Hermitian generators, the Killing form given by $\mathrm{Tr}$ is positive-definite.

By promoting $\phi$ to $\Phi$, we find that the supersymmetric completion of $\mathcal{L}_{0}$ is

$$
\tilde{\mathcal{L}}_{0}=\int d^{2} \theta K=\frac{i\left(\phi \dot{\phi}^{\dagger}-\phi^{\dagger} \dot{\phi}\right)}{1+|\phi|^{2}}-\frac{\psi^{\dagger} \psi}{\left(1+|\phi|^{2}\right)^{2}}, \quad K \equiv \log \left(1+|\Phi|^{2}\right) .
$$

We have covered $\mathbb{C P}^{1}$ with patches having local coordinates $\Phi$ and $1 / \Phi$, so that $K$ is the Kähler potential for the Fubini-Study metric in the patch containing the origin.

To gauge $\tilde{\mathcal{L}}_{0}$ in a supersymmetric way and thereby obtain the supersymmetric completion of $\mathcal{L}$ requires promoting the $A_{i}$ to $V_{i}$, which is more involved. Having eliminated the 
integration variable $g$ in favor of $\phi$, let us denote by $g$ what we called $h_{\ell}$ in (3.10). Writing finite and infinitesimal local $\mathrm{SU}(2)$ transformations as

$$
g=\left(\begin{array}{cc}
a & b \\
-\bar{b} & \bar{a}
\end{array}\right) \sim\left(\begin{array}{cc}
1+\frac{i \epsilon_{3}}{2} & \frac{i \epsilon_{1}+\epsilon_{2}}{2} \\
\frac{i \epsilon_{1}-\epsilon_{2}}{2} & 1-\frac{i \epsilon_{3}}{2}
\end{array}\right),
$$

finite and infinitesimal gauge transformations take the form

$$
\begin{aligned}
A \rightarrow g A g^{-1}-i \dot{g} g^{-1} & \Longleftrightarrow \delta_{\mathrm{SU}(2)} A_{i}=\epsilon_{i j k} A_{j} \epsilon_{k}+\dot{\epsilon}_{i}, \\
\Phi \rightarrow \frac{a \Phi+b}{-\bar{b} \Phi+\bar{a}} & \Longleftrightarrow \delta_{\mathrm{SU}(2)} \Phi=\epsilon_{i} X_{i},\left(X_{1}, X_{2}, X_{3}\right) \equiv \frac{1}{2}\left(i\left(1-\Phi^{2}\right), 1+\Phi^{2}, 2 i \Phi\right),
\end{aligned}
$$

where the holomorphic SU(2) Killing vectors $X_{i}$ satisfy $\left[X_{i} \partial_{\Phi}, X_{j} \partial_{\Phi}\right]=\epsilon_{i j k} X_{k} \partial_{\Phi}$. Then

$$
\delta_{\mathrm{SU}(2)} K=\epsilon_{i}\left(\mathcal{F}_{i}+\overline{\mathcal{F}}_{i}\right), \quad\left(\mathcal{F}_{1}, \mathcal{F}_{2}, \mathcal{F}_{3}\right) \equiv \frac{1}{2}(-i \Phi, \Phi, i)
$$

(any purely imaginary $\mathcal{F}_{3}$ would do, but our choice leads to the "canonical" Noether currents transforming in the adjoint representation). To implement the Noether procedure, we promote the real $\epsilon_{i}$ to complex chiral superfields $\Lambda_{i}$ :

$$
\delta_{\mathrm{SU}(2)} \Phi=\Lambda_{i} X_{i}
$$

The corresponding change in $\tilde{\mathcal{L}}_{0}$ can be read off from

$$
\delta_{\mathrm{SU}(2)} K=\Lambda_{i} \mathcal{F}_{i}+\bar{\Lambda}_{i} \overline{\mathcal{F}}_{i}-i\left(\Lambda_{i}-\bar{\Lambda}_{i}\right) J_{i}
$$

where the SU(2) Noether currents (Killing potentials) are the real superfields

$$
J_{i}=\frac{i X_{i} \Phi^{\dagger}}{1+|\Phi|^{2}}-i \mathcal{F}_{i} \Longrightarrow\left(J_{1}, J_{2}, J_{3}\right)=\frac{1}{2}\left(-\frac{\Phi+\Phi^{\dagger}}{1+|\Phi|^{2}},-\frac{i\left(\Phi-\Phi^{\dagger}\right)}{1+|\Phi|^{2}}, \frac{1-|\Phi|^{2}}{1+|\Phi|^{2}}\right)
$$

which satisfy $J_{i}^{2}=1 / 4$ and

$$
\delta_{\mathrm{SU}(2)} J_{i}=-\frac{1}{2} \epsilon_{i j k}\left(\Lambda_{j}+\bar{\Lambda}_{j}\right) J_{k}+i\left(\Lambda_{j}-\bar{\Lambda}_{j}\right) J_{j} J_{i}-\frac{i}{4}\left(\Lambda_{i}-\bar{\Lambda}_{i}\right) .
$$

This generalizes $\delta_{\mathrm{SU}(2)} J_{i}=-\epsilon_{i j k} \epsilon_{j} J_{k}$ for real $\epsilon_{i}$. Now, if we could find a counterterm $\Gamma$ such that $\delta_{\mathrm{SU}(2)} \Gamma=i\left(\Lambda_{i}-\bar{\Lambda}_{i}\right) J_{i}$, then we would be done: the supersymmetric completion of $\mathcal{L}$ would be the minimally gauged supersymmetric $\mathbb{C P} P^{1}$ model $\tilde{\mathcal{L}}=\tilde{\mathcal{L}}_{0}+\tilde{\mathcal{L}}_{A}$ where

$$
\tilde{\mathcal{L}}_{A}=\int d^{2} \theta \Gamma, \quad \delta_{\mathrm{SU}(2)} \Gamma=i\left(\Lambda_{i}-\bar{\Lambda}_{i}\right) J_{i}
$$

Note that $\tilde{\mathcal{L}}$ is invariant under local $\mathrm{SU}(2)$ because, in light of (4.15), the total variation of $K+\Gamma$ takes the form of a Kähler transformation. There exists a standard procedure for constructing such a $\Gamma$ [41], which we review in appendix B.1. Its exact form is

$$
\Gamma=2 \int_{0}^{1} d \alpha e^{i \alpha V_{i} O_{i}} V_{j} J_{j}
$$


where $O_{i}=X_{i} \partial_{\Phi}-\bar{X}_{i} \partial_{\Phi^{\dagger}}$. For our purposes, it suffices to work in Wess-Zumino gauge, where the bulk vector superfield is nilpotent of degree three $\left(V_{3 \mathrm{D}}^{3}=0\right)$ and its restriction to the line is nilpotent of degree two $\left(V_{1 \mathrm{D}}^{2}=0\right)$ : namely, $V_{i}=\theta \theta^{\dagger} A_{i}$. In this gauge, we have $\Gamma=2 V_{i} J_{i}$, so that $\tilde{\mathcal{L}}$ reduces to the non-manifestly supersymmetric Lagrangian $\tilde{\mathcal{L}}_{0}+\mathcal{L}_{A}$. In arbitrary gauge, $\tilde{\mathcal{L}}$ contains terms of arbitrarily high order in the dimensionless bottom component of $V$.

An important point is the following. There are two standard ways of geometrizing the action of SU(2) on $S^{2}$, both of which can be found in the literature. These two conventions differ by signs, leading to slightly different SU(2) Noether currents. First, the action of $\mathrm{SU}(2)$ on $S^{2}$ descends from the adjoint action of $\mathrm{SU}(2)$ on $\mathfrak{s u}(2) \cong \mathbb{R}^{3}$, which preserves the Killing form (hence $S^{2} \subset \mathbb{R}^{3}$ ). This convention is used in, e.g., [41], corresponding to

$$
\left(J_{1}, J_{2}, J_{3}\right)_{\text {other }}=\frac{1}{2}\left(\frac{\Phi+\Phi^{\dagger}}{1+|\Phi|^{2}},-\frac{i\left(\Phi-\Phi^{\dagger}\right)}{1+|\Phi|^{2}},-\frac{1-|\Phi|^{2}}{1+|\Phi|^{2}}\right)
$$

(with the relative sign of $J_{2}$ reversed relative to our (4.16)). Second, $\mathrm{SU}(2)$ acts on $\mathbb{C P}^{1}$ by linear fractional transformations. We use the latter convention unless stated otherwise. For further details, see appendix C.1.

\subsubsection{Effective action}

To compute the effective action generated by integrating out $\psi$, we add an $\mathrm{SU}(2)$-invariant kinetic term for $\psi$ (with an implicit dimensionful coefficient) as a UV regulator:

$$
\mathcal{L}^{\prime}=\int d^{2} \theta K^{\prime}=-\frac{i\left(\psi^{\dagger} \dot{\psi}-\dot{\psi}^{\dagger} \psi\right)+4 \dot{\phi} \dot{\phi}^{\dagger}}{\left(1+|\phi|^{2}\right)^{2}}-\frac{2 i\left(\dot{\phi}^{\dagger} \phi-\phi^{\dagger} \dot{\phi}\right) \psi^{\dagger} \psi}{\left(1+|\phi|^{2}\right)^{3}}, \quad K^{\prime} \equiv \frac{D^{\dagger} \Phi^{\dagger} D \Phi}{\left(1+|\Phi|^{2}\right)^{2}} .
$$

Note that since $D \Phi=\psi-2 i \theta^{\dagger} \dot{\phi}+i \theta \theta^{\dagger} \dot{\psi}$ transforms in the same way under $\mathrm{SU}(2)$ as its bottom component $\psi, K^{\prime}$ is automatically invariant under global $\mathrm{SU}(2)$. We want to gauge $K^{\prime}$. With chiral superfield gauge transformation parameters, we have (note $D X_{i}=2 \mathcal{F}_{i} D \Phi$ )

$$
\delta_{\mathrm{SU}(2)} K^{\prime}=-i\left(\Lambda_{i}-\Lambda_{i}^{\dagger}\right) J_{i}^{\prime}-i\left(D \Lambda_{i} I_{i}-D^{\dagger} \Lambda_{i}^{\dagger} I_{i}^{\dagger}\right)
$$

where $J_{i}^{\prime}$ are the bosonic Noether currents associated to $K^{\prime}$ and the $I_{i}$ are fermionic:

$$
J_{i}^{\prime}=-2 K^{\prime} J_{i}, \quad I_{i}=\frac{i X_{i}(D \Phi)^{\dagger}}{\left(1+|\Phi|^{2}\right)^{2}} .
$$

There exists a counterterm $\Gamma^{\prime}$ satisfying

$$
\delta_{\mathrm{SU}(2)} \Gamma^{\prime}=i\left(\Lambda_{i}-\bar{\Lambda}_{i}\right) J_{i}^{\prime}+i\left(D \Lambda_{i} I_{i}-D^{\dagger} \bar{\Lambda}_{i} \bar{I}_{i}\right),
$$

which takes the form

$$
\int d^{2} \theta \Gamma^{\prime}=\frac{2 \psi^{\dagger} \psi}{\left(1+|\phi|^{2}\right)^{2}}\left[\frac{\left(A_{1}+i A_{2}\right) \phi+\left(A_{1}-i A_{2}\right) \phi^{\dagger}-A_{3}\left(1-|\phi|^{2}\right)}{1+|\phi|^{2}}\right]+\cdots
$$

in Wess-Zumino gauge, such that the Lagrangian

$$
\tilde{\mathcal{L}}^{\prime}=\int d^{2} \theta\left(K^{\prime}+\Gamma^{\prime}\right) \equiv \mathcal{L}_{\psi}-\frac{4 \dot{\phi} \dot{\phi}^{\dagger}}{\left(1+|\phi|^{2}\right)^{2}}+\cdots
$$


(written in Wess-Zumino gauge) is invariant under local $\mathrm{SU}(2)$, where

$$
\mathcal{L}_{\psi}=-\frac{i\left(\psi^{\dagger} \dot{\psi}-\dot{\psi}^{\dagger} \psi\right)}{\left(1+|\phi|^{2}\right)^{2}}-\frac{2 \mathcal{L} \psi^{\dagger} \psi}{\left(1+|\phi|^{2}\right)^{2}}
$$

is itself invariant under local $\mathrm{SU}(2)$ (we construct $\Gamma^{\prime}$ in appendix B.2 using a general prescription for the full nonlinear gauging of supersymmetric sigma models with higherderivative terms). Thus the "..." in $\tilde{\mathcal{L}}^{\prime}$ contains only dimension-two terms not involving $\psi$, namely the couplings to $A_{i}$ necessary to make the two-derivative term in $\phi$ invariant under local SU(2). Making the scale $\mu$ of the higher-dimension terms explicit, consider

$$
\tilde{\mathcal{L}}_{\text {tot }}=j \tilde{\mathcal{L}}-\frac{1}{2 \mu} \tilde{\mathcal{L}}^{\prime} \equiv j \mathcal{L}+\psi^{\dagger} \mathcal{D} \psi+\frac{2 \dot{\phi} \dot{\phi}^{\dagger}}{\mu\left(1+|\phi|^{2}\right)^{2}}+\cdots,
$$

where we have integrated by parts. Performing the path integral over $\psi$ (as in appendix C.2) generates the one-loop effective action

$$
\operatorname{tr} \log \mathcal{D}= \pm \frac{i}{2} \int d t \mathcal{L}
$$

The regularization-dependent sign is fixed to "-" by canonical quantization, leading to a shift $j \rightarrow j-1 / 2$. The “..." terms in $\tilde{\mathcal{L}}^{\prime}$ decouple at low energies $(\mu \rightarrow \infty)$.

\subsection{Shift from canonical quantization}

Canonical quantization of the $\mathcal{N}=2$ quantum mechanics provides another perspective on the shift in $j$. Here, we set $A_{i}=0$, whence

$$
\left.\tilde{\mathcal{L}}\right|_{A_{i}=0}=\tilde{\mathcal{L}}_{0},\left.\quad \tilde{\mathcal{L}}^{\prime}\right|_{A_{i}=0}=\mathcal{L}^{\prime}
$$

so that the full Lagrangian is $\left.\tilde{\mathcal{L}}_{\text {tot }}\right|_{A_{i}=0}=j \tilde{\mathcal{L}}_{0}-\frac{1}{2 \mu} \mathcal{L}^{\prime}=L_{B}+L_{F}$ where $L_{B}$ and $L_{F}$ describe 1D sigma models with $S^{2}$ target space:

$$
\begin{aligned}
& L_{B}=\frac{i j\left(\phi \dot{\phi}^{\dagger}-\phi^{\dagger} \dot{\phi}\right)}{1+|\phi|^{2}}+\frac{i \alpha}{2}\left(\frac{\dot{\phi}}{\phi}-\frac{\dot{\phi}^{\dagger}}{\phi^{\dagger}}\right)+\frac{2 \dot{\phi} \dot{\phi}^{\dagger}}{\mu\left(1+|\phi|^{2}\right)^{2}}, \\
& L_{F}=-\left[j-\frac{i\left(\phi \dot{\phi}^{\dagger}-\phi^{\dagger} \dot{\phi}\right)}{\mu\left(1+|\phi|^{2}\right)}\right] \frac{\psi^{\dagger} \psi}{\left(1+|\phi|^{2}\right)^{2}}+\frac{i\left(\psi^{\dagger} \dot{\psi}-\dot{\psi}^{\dagger} \psi\right)}{2 \mu\left(1+|\phi|^{2}\right)^{2}}
\end{aligned}
$$

For later convenience, we have added a total derivative, parametrized by $\alpha \in \mathbb{R}$, to $L_{B}$. Its meaning is as follows: $L_{B}$ describes an electrically charged particle on $S^{2}$ in the field of a magnetic monopole of charge $\propto j$ at the center, with the scale $\mu \in \mathbb{R}_{\geq 0}$ (the spectral gap) proportional to its inverse mass and $\alpha$ parametrizing the longitudinal gauge of the monopole vector potential. We define the gauges $S, E$, and $N$ by setting $\alpha=(0, j, 2 j)$, respectively. We refer to $L_{B}$ as the "bosonic system" and to $L_{B}+L_{F}$ as the corresponding "supersymmetric system." We now summarize the results of quantizing the theories $L_{B}$ and $L_{B}+L_{F}$ : details are given in appendix C.3. As when computing the effective action, we use the $1 / \mu$ terms as a technical aid; they have the effect of enlarging the phase space. 


\subsubsection{Bosonic system}

As a warmup, consider $L_{B}$ alone. At finite $\mu$, the phase space is $(2+2)$-dimensional and the quantum Hamiltonian can be written as

$$
H_{j}=\frac{\mu}{2}\left(\vec{L}^{2}-j^{2}\right)=\frac{\mu}{2}\left(\ell(\ell+1)-j^{2}\right) .
$$

Here, $\vec{L}^{2}=\frac{1}{2}\left(L_{+} L_{-}+L_{-} L_{+}\right)+L_{3}^{2}$ and we have defined the operators

$$
\begin{aligned}
& L_{+}=-\phi^{2} \frac{\partial}{\partial \phi}-\frac{\partial}{\partial \phi^{\dagger}}+\frac{2 j|\phi|^{2}+\alpha\left(1-|\phi|^{2}\right)}{2|\phi|^{2}} \phi, \\
& L_{-}=\frac{\partial}{\partial \phi}+\left(\phi^{\dagger}\right)^{2} \frac{\partial}{\partial \phi^{\dagger}}+\frac{2 j|\phi|^{2}+\alpha\left(1-|\phi|^{2}\right)}{2|\phi|^{2}} \phi^{\dagger}, \\
& L_{3}=\phi \frac{\partial}{\partial \phi}-\phi^{\dagger} \frac{\partial}{\partial \phi^{\dagger}}-(j-\alpha),
\end{aligned}
$$

which satisfy $\left[L_{3}, L_{ \pm}\right]= \pm L_{ \pm},\left[L_{+}, L_{-}\right]=2 L_{3}$. The spectrum is constrained to $\ell \geq j$ by an $L_{3}$ selection rule, with each level $\ell$ appearing once; the eigenfunctions of the associated generalized angular momentum are monopole spherical harmonics. As $\mu \rightarrow \infty$, all states except those with $\ell=j$ decouple. Rather than taking the decoupling limit $\mu \rightarrow \infty$ in $L_{B}$, which projects out all but the spin- $j$ states, setting $j=0$ yields the rigid rotor. Its Hamiltonian is given in terms of the Laplace-Beltrami operator $\Delta_{S^{2}}$, whose spectrum is $-\ell(\ell+1)$ with degeneracy $2 \ell+1$ for $\ell \geq 0$.

The bosonic theory with $\mu=\infty\left(L_{B}=j \mathcal{L}_{0}\right.$, in $S$ gauge $)$ is the well-known WessZumino term for quantization of spin. The action computes the solid angle enclosed by a trajectory on the sphere, and the Dirac quantization condition requires that the coefficient $j$ be a half-integer. Quantizing the compact phase space $S^{2}$ yields $2 j+1$ states $|j, m\rangle$, all eigenstates of $L_{3}$. Indeed, at $\mu=\infty$, the phase space is $(1+1)$-dimensional and we can write

$$
L_{+}=-\phi^{2} \partial_{\phi}+(2 j-\alpha) \phi, \quad L_{-}=\partial_{\phi}+\frac{\alpha}{\phi}, \quad L_{3}=\phi \partial_{\phi}-(j-\alpha) .
$$

The wavefunctions are $\phi^{-\alpha}, \ldots, \phi^{2 j-\alpha}$, the eigenvalues range from $-j$ to $j$ in integer steps, and $\vec{L}^{2}=j(j+1)$.

\subsubsection{Supersymmetric system}

For $L_{B}+L_{F}$, let us keep $\mu$ finite (work in the full phase space) and set $\alpha=0$. Write

$$
\chi=\frac{\psi}{\sqrt{\mu}\left(1+|\phi|^{2}\right)},
$$

which satisfies $\left\{\chi, \chi^{\dagger}\right\}=1$ upon quantization. The supercharges are represented by differential operators as

$$
Q=\psi\left(\frac{\partial}{\partial \phi}-\frac{(j+1 / 2) \phi^{\dagger}}{1+|\phi|^{2}}\right), \quad Q^{\dagger}=\psi^{\dagger}\left(-\frac{\partial}{\partial \phi^{\dagger}}-\frac{(j-1 / 2) \phi}{1+|\phi|^{2}}\right)
$$


which are adjoints with respect to the Fubini-Study measure. The Hamiltonian is

$$
H^{\prime}=\frac{1}{2}\left\{Q, Q^{\dagger}\right\}=H_{j+\chi^{\dagger} \chi-1 / 2}+j \mu \chi^{\dagger} \chi-\frac{\mu}{2}(j-1 / 2)=\frac{\mu}{2}\left(\vec{L}_{f}^{2}-(j+1 / 2)(j-1 / 2)\right)
$$

where $\vec{L}_{f}=\left.\vec{L}\right|_{j+\chi^{\dagger} \chi-1 / 2}$. On the Hilbert space $\left(L^{2}\left(S^{2}, \mathbb{C}\right) \otimes|0\rangle\right) \oplus\left(L^{2}\left(S^{2}, \mathbb{C}\right) \otimes \chi^{\dagger}|0\rangle\right)$,

$$
\begin{aligned}
H^{\prime} & =\left(\begin{array}{cc}
H_{j-1 / 2}-\mu(j-1 / 2) / 2 & 0 \\
0 & H_{j+1 / 2}+\mu(j+1 / 2) / 2
\end{array}\right) \\
& =\frac{\mu}{2}\left(\begin{array}{cc}
\ell_{b}\left(\ell_{b}+1\right)-(j-1 / 2)(j+1 / 2) & 0 \\
0 & \ell_{f}\left(\ell_{f}+1\right)-(j-1 / 2)(j+1 / 2)
\end{array}\right)
\end{aligned}
$$

where $\ell_{b} \geq j-1 / 2$ and $\ell_{f} \geq j+1 / 2$. There are $2 j$ bosonic ground states at $\ell_{b}=j-1 / 2$. This fixes the sign of the previous path integral calculation. As a further check, the quantum representations of the fermionic monopole angular momenta $\left(L_{f}\right)_{i}$ are presented in (C.29). Their classical counterparts (C.41) reduce to the classical $L_{i}$ with $j-1 / 2$ as $\mu \rightarrow \infty$.

\subsection{Shift from 1D supersymmetric index}

To make contact with bulk Wilson loops, we compute both the non-supersymmetric twisted partition function and the flavored Witten index

$$
I_{\mathcal{N}=0}=\operatorname{Tr}\left(e^{-\beta H} e^{i z L_{3}}\right), \quad I_{\mathcal{N}=2}=\operatorname{Tr}\left[(-1)^{F} e^{-\beta H^{\prime}} e^{i z\left(L_{f}\right)_{3}}\right]
$$

by working semiclassically in the Euclidean path integral. Let

$$
\begin{aligned}
& L_{B, E}=\frac{j\left(\phi \dot{\phi}^{\dagger}-\phi^{\dagger} \dot{\phi}\right)}{1+|\phi|^{2}}+\frac{\alpha}{2}\left(\frac{\dot{\phi}}{\phi}-\frac{\dot{\phi}^{\dagger}}{\phi^{\dagger}}\right)+\frac{2 \dot{\phi} \dot{\phi}^{\dagger}}{\mu\left(1+|\phi|^{2}\right)^{2}}, \\
& L_{F, E}=\left[j+\frac{\phi \dot{\phi}^{\dagger}-\phi^{\dagger} \dot{\phi}}{\mu\left(1+|\phi|^{2}\right)}\right] \frac{\psi^{\dagger} \psi}{\left(1+|\phi|^{2}\right)^{2}}+\frac{\psi^{\dagger} \dot{\psi}-\dot{\psi}^{\dagger} \psi}{2 \mu\left(1+|\phi|^{2}\right)^{2}}
\end{aligned}
$$

denote the Euclideanized versions of $L_{B}$ and $L_{F}$, with dots denoting $\tau$-derivatives. Then

$$
I_{\mathcal{N}=0}=\int D \phi^{\dagger} D \phi e^{-\int_{0}^{\beta} d \tau L_{B, E}}, \quad I_{\mathcal{N}=2}=\int D \phi^{\dagger} D \phi D \psi^{\dagger} D \psi e^{-\int_{0}^{\beta} d \tau\left(L_{B, E}+L_{F, E}\right)},
$$

with boundary conditions twisted by $e^{i z L_{3}}$ or $e^{i z\left(L_{f}\right)_{3}}$ as appropriate. While both $I_{\mathcal{N}=0}$ and $I_{\mathcal{N}=2}$ are known from canonical quantization, our goal here is to introduce the localization argument via what amounts to a derivation of the Weyl character formula (1.2) as a sum of two terms coming from the classical saddle points with a spin-independent prefactor coming from the one-loop determinants.

We first compute $I_{\mathcal{N}=0}$ in the bosonic problem. Set $\mu=\infty$ and work in the $E$ gauge (not to be confused with " $E$ for Euclidean"), where

$$
L_{B, E}=\frac{j\left(\phi \dot{\phi}^{\dagger}-\phi^{\dagger} \dot{\phi}\right)}{1+|\phi|^{2}}+\frac{j}{2} \partial_{\tau} \log \left(\frac{\phi}{\phi^{\dagger}}\right) .
$$


We restrict the path integral to field configurations satisfying $\phi(\tau+\beta)=e^{i z} \phi(\tau)$, for which

$$
\int_{0}^{\beta} d \tau \partial_{\tau} \log \left(\frac{\phi}{\phi^{\dagger}}\right)=2 i z
$$

With this restriction, the action is extremized when $\phi=\phi_{\mathrm{cl}} \in\{0, \infty\}$ (the two fixed points of the $L_{3}$ action). We see that $\left.L_{B, E}\right|_{0}=i j z / \beta$ and $\left.L_{B, E}\right|_{\infty}=-i j z / \beta$. First expand around $\phi_{\mathrm{cl}}=0$ with perturbation $\Delta: \phi=\phi_{\mathrm{cl}}+\Delta=\Delta$, where $\Delta$ satisfies the twisted boundary condition. Its mode expansion takes the form

$$
\Delta=\frac{1}{\sqrt{\beta}} \sum_{n=-\infty}^{\infty} \Delta_{n} e^{i(2 \pi n+z) \tau / \beta}
$$

from which we obtain simply

$$
\left.\int_{0}^{\beta} d \tau L_{B, E}\right|_{O\left(\Delta^{2}\right)}=j \int_{0}^{\beta} d \tau\left(\Delta \dot{\Delta}^{\dagger}-\Delta^{\dagger} \dot{\Delta}\right)=-\frac{2 i j}{\beta} \sum_{n=-\infty}^{\infty}(2 \pi n+z)\left|\Delta_{n}\right|^{2} .
$$

Thus the one-loop factor from expanding around $\phi_{\mathrm{cl}}=0$ is

$$
\left.Z_{1-\text { loop }}\right|_{0}=\exp \left[-\sum_{n=-\infty}^{\infty} \log (2 \pi n+z)\right]=\frac{e^{a z+b}}{\sin (z / 2)}=-\frac{e^{-i z / 2}}{2 i \sin (z / 2)}
$$

where the integration constants $a, b$ parametrize the counterterms by which different regularization schemes differ. We present several ways to fix the values of $a$ and $b$ to those written above. First, (4.49) is the only choice consistent with canonical quantization. Second, Hurwitz zeta function regularization yields

$$
\sum_{n=-\infty}^{\infty} \log (A n+B)=\left.\log \left(1-e^{2 \pi i B / A}\right) \Longrightarrow Z_{1-\operatorname{loop}}\right|_{0}=\frac{1}{1-e^{i z}}=-\frac{e^{-i z / 2}}{2 i \sin (z / 2)}
$$

Third, performing free-field subtraction (normalizing the functional determinant, sans zero mode) at finite $\mu$ and then taking $\mu \rightarrow \infty$ yields the same answer. Indeed, accounting for the $1 / \mu$ term in (4.42), the kinetic operator for bosonic fluctuations $\Delta$ is $-2\left(j \partial_{\tau}+\partial_{\tau}^{2} / \mu\right)$ where the eigenvalues of $\partial_{\tau}$ are $i(2 \pi n+z) / \beta$, giving the regularized product

$$
\left.Z_{1 \text {-loop }}\right|_{0}=\frac{1}{\operatorname{det}\left(j \partial_{\tau}+\partial_{\tau}^{2} / \mu\right)}=-\frac{\sinh (\beta \mu j / 2)}{2 i \sin (z / 2) \sinh ((\beta \mu j+i z) / 2)} \stackrel{\beta \mu \rightarrow \infty}{\longrightarrow}-\frac{e^{-(j /|j|) i z / 2}}{2 i \sin (z / 2)}
$$

Now note that taking $\phi \rightarrow 1 / \phi$ leaves $L_{B, E}$ in $E$ gauge (4.45) invariant (with the $1 / \mu$ term in (4.42) being invariant by itself) while taking $z \rightarrow-z$ in the boundary condition for the path integral. Hence

$$
\left.Z_{1 \text {-loop }}\right|_{\infty}=\left.\left(\left.Z_{1-\text { loop }}\right|_{0}\right)\right|_{z \rightarrow-z}=\frac{e^{i z / 2}}{2 i \sin (z / 2)}
$$


and it follows that

$$
I_{\mathcal{N}=0}=\sum_{0, \infty} e^{-\beta L_{B, E}} Z_{1 \text {-loop }}=\frac{e^{i(j+1 / 2) z}-e^{-i(j+1 / 2) z}}{2 i \sin (z / 2)}=\frac{\sin ((j+1 / 2) z)}{\sin (z / 2)} .
$$

This is, of course, a special case of the Duistermaat-Heckman formula for longitudinal rotations of $S^{2}$, with the contribution from each fixed point weighted by the appropriate sign. As a consequence, the index is an even function of $z$ (invariant under the Weyl group $\mathbb{Z}_{2}$ ), as it must be, because the Hilbert space splits into representations of $\mathrm{SU}(2) .{ }^{13}$

We now compute $I_{\mathcal{N}=2}$, keeping $\mu$ finite. In the supersymmetric problem, the $E$ gauge corresponds to choosing the Kähler potential $\log \left(1+|\Phi|^{2}\right)-\frac{1}{2} \log |\Phi|^{2}$, which is invariant under $\Phi \rightarrow 1 / \Phi$. In component fields, the Lagrangian is $L_{B, E}+L_{F, E}$ with $\alpha=j$. Expanding in both bosonic fluctuations $\Delta$ and fermionic fluctuations $\Xi\left(\psi=\psi_{\mathrm{cl}}+\Xi=\Xi\right)$ gives

$$
\left.\left(L_{B, E}+L_{F, E}\right)\right|_{O\left(\Delta^{2}+\Xi^{2}\right)}=j\left(\Delta \dot{\Delta}^{\dagger}-\Delta^{\dagger} \dot{\Delta}+\Xi^{\dagger} \Xi\right)+\frac{2}{\mu} \dot{\Delta} \dot{\Delta}^{\dagger}+\frac{1}{2 \mu}\left(\Xi^{\dagger} \dot{\Xi}-\dot{\Xi}^{\dagger} \Xi\right) .
$$

The part of the Lagrangian quadratic in fluctuations, as written above, is supersymmetric by itself. ${ }^{14}$ Twisted boundary conditions in the path integral are implemented by $\left(L_{f}\right)_{3}$, which satisfies $\left[\left(L_{f}\right)_{3}, \phi\right]=\phi$ and $\left[\left(L_{f}\right)_{3}, \psi\right]=\psi$. The moding for the fermionic fluctuations

$$
\Xi=\frac{1}{\sqrt{\beta}} \sum_{n=-\infty}^{\infty} \Xi_{n} e^{i(2 \pi n+z) \tau / \beta}
$$

is integral because at $z=0$, the insertion of $(-1)^{F}$ would require periodic boundary conditions for fermions on the thermal circle. Hence the fermions contribute a factor of

$$
\exp \left[\sum_{n=-\infty}^{\infty} \log \left(\frac{2 \pi n+z}{\beta \mu}-i j\right)\right]
$$

to $\left.Z_{1 \text {-loop }}\right|_{0}$ (to obtain a nontrivial functional determinant, we cannot neglect the fermion kinetic term, which is why we have kept $\mu$ finite). Hurwitz zeta function regularization alone does not suffice for taking the $\beta \mu \rightarrow \infty$ limit, so we instead perform free-field subtraction (divide by a fiducial functional determinant):

$$
\operatorname{det}\left(j+\partial_{\tau} / \mu\right)=\prod_{n=-\infty}^{\infty} \frac{(2 \pi n+z) / \beta \mu-i j}{2 \pi n / \beta \mu-i j}=\frac{\sin ((i \beta \mu j-z) / 2)}{\sin (i \beta \mu j / 2)} \stackrel{\beta \mu \rightarrow \infty}{\longrightarrow} e^{(j /|j|) i z / 2} .
$$

\footnotetext{
${ }^{13}$ That the index is even in $z$, as implied by canonical quantization, fixes potential multiplicative ambiguities in the path integral computation. For example, regardless of $\alpha$ in (4.42), $L_{3}$ in (4.34) satisfies $\left[L_{3}, \phi\right]=\phi$ and hence implements the same twisted boundary condition $\phi(\tau+\beta)=e^{i z} \phi(\tau)$. However, to obtain an answer that is even in $z$ requires implementing the boundary condition using the operator $\left.L_{3}\right|_{\alpha=j}$. In this way, the constant shift in $L_{3}$ relative to $\left.L_{3}\right|_{\alpha=j}$ gives an overall phase of $e^{-i(j-\alpha) z}$, which combines with the classical contributions $\left.e^{-\beta L_{B, E}}\right|_{0}=e^{-i \alpha z}$ and $\left.e^{-\beta L_{B, E}}\right|_{\infty}=e^{i(2 j-\alpha) z}$ to produce the gauge-independent result (4.53). To avoid this complication, we have chosen to work in the $E$ gauge from the beginning.

${ }^{14}$ We have that

$$
\delta\left(\Delta \dot{\Delta}^{\dagger}-\Delta^{\dagger} \dot{\Delta}+\Xi^{\dagger} \Xi\right)=-\partial_{\tau}\left(\epsilon \Xi \Delta^{\dagger}+\epsilon^{\dagger} \Xi^{\dagger} \Delta\right), \quad \delta\left[2 \dot{\Delta} \dot{\Delta}^{\dagger}+\frac{1}{2}\left(\Xi^{\dagger} \dot{\Xi}-\dot{\Xi}^{\dagger} \Xi\right)\right]=\partial_{\tau}\left(\epsilon \Xi^{\dagger} \dot{\Delta}^{\dagger}-\epsilon^{\dagger} \Xi^{\dagger} \dot{\Delta}\right)
$$

under the (global) Euclidean SUSY variations $(\delta \Delta, \delta \Xi)=\left(\epsilon \Xi, 2 \epsilon^{\dagger} \dot{\Delta}\right)$ and $\left(\delta \Delta^{\dagger}, \delta \Xi^{\dagger}\right)=\left(-\epsilon^{\dagger} \Xi^{\dagger},-2 \epsilon \dot{\Delta}^{\dagger}\right)$.
} 
Taking $j$ positive, this reduces to a phase of $e^{i z / 2}$. By similar reasoning to that in the bosonic case, we conclude that

$$
I_{\mathcal{N}=2}=\frac{\sin (j z)}{\sin (z / 2)}
$$

Again, this is the only answer consistent with canonical quantization. Thus in the supersymmetric theory, the one-loop shift of $j$ due to the bosons $(+1 / 2)$ exactly cancels that due to the fermions $(-1 / 2)$.

\subsubsection{Localization in $1 \mathrm{D}$}

In both the bosonic and supersymmetric theories, direct comparison to canonical quantization shows that the semiclassical (one-loop) approximation for the index is exact. It is natural to ask why this should be so, and supersymmetry provides an answer. While the exactness in the bosonic case can only be heuristically justified by the Dirac quantization condition on $j$, it can be rigorously justified by appealing to the supersymmetric case.

In its most basic form, the localization principle starts from the fact that a Euclidean partition function deformed by a total variation of some nilpotent symmetry $\delta\left(\delta^{2}=0\right)$ of both the action and the measure is independent of the coefficient of this deformation:

$$
Z(t)=\int \mathcal{D} \Phi e^{-S[\Phi]+t \delta V} \Longrightarrow \frac{d Z(t)}{d t}=\int \mathcal{D} \Phi \delta\left(e^{-S[\Phi]+t \delta V} V\right)=0
$$

If the bosonic part of $\delta V$ is positive-semidefinite, then as $t \rightarrow \infty$, the path integral localizes to $\delta V=0$. For a given field configuration with $\delta V=0$, one can compute a semiclassical path integral for fluctuations on top of this background, and then integrate over all such backgrounds to obtain the exact partition function.

In our case, the quadratic terms arising from perturbation theory are already $\left(Q+Q^{\dagger}\right)$ exact, without the need to add any localizing terms. Indeed, we compute that ${ }^{15}$

$$
\begin{aligned}
\delta\left(\delta\left(\phi^{\dagger} \phi\right)\right) & =2 \epsilon^{\dagger} \epsilon\left(\phi \dot{\phi}^{\dagger}-\phi^{\dagger} \dot{\phi}+\psi^{\dagger} \psi\right), \\
\delta\left(\delta\left(\psi^{\dagger} \psi\right)\right) & =2 \epsilon^{\dagger} \epsilon\left(4 \dot{\phi} \dot{\phi}^{\dagger}+\psi^{\dagger} \dot{\psi}-\dot{\psi}^{\dagger} \psi\right) .
\end{aligned}
$$

Up to overall factors, these are precisely the quadratic expressions (4.54) that we integrate over the fluctuations $\Delta, \Xi$ to compute the one-loop factors in the index $I_{\mathcal{N}=2}$. As we take the coefficient of either the $\delta\left(\delta\left(\phi^{\dagger} \phi\right)\right)$ term or the $\delta\left(\delta\left(\psi^{\dagger} \psi\right)\right)$ term to infinity, the original Lagrangian $L_{B, E}+L_{F, E}$ becomes irrelevant for the one-loop analysis, but since these terms have the same critical points as the original Lagrangian, the result of the localization analysis coincides with that of the original Lagrangian, proving that the path integral for the latter is one-loop exact. ${ }^{16}$ Furthermore, the final result is independent of the coefficient of either term. This has a simple explanation: the regularized bosonic and

\footnotetext{
${ }^{15}$ Here, we again use that in Euclidean signature,$$
\delta \phi=\delta_{\epsilon} \phi=\epsilon \psi, \quad \delta \phi^{\dagger}=\delta_{\epsilon^{\dagger}} \phi^{\dagger}=-\epsilon^{\dagger} \psi^{\dagger}, \quad \delta \psi=\delta_{\epsilon^{\dagger}} \psi=2 \epsilon^{\dagger} \dot{\phi}, \quad \delta \psi^{\dagger}=\delta_{\epsilon} \psi^{\dagger}=-2 \epsilon \dot{\phi}^{\dagger}
$$

where $\delta \mathcal{O} \equiv\left[\epsilon Q+\epsilon^{\dagger} Q^{\dagger}, \mathcal{O}\right]$ and $\delta_{\epsilon, \epsilon^{\dagger}}$ are Grassmann-even.

${ }^{16}$ Note that the bosonic part of the $\delta\left(\delta\left(\phi^{\dagger} \phi\right)\right)$ term is not positive-semidefinite; indeed, it is imaginary. We are implicitly using a stationary phase argument.
} 
fermionic functional determinants (4.51) and (4.57) have a product which is independent of $\beta \mu$, namely

$$
\frac{\operatorname{det}\left(j+\partial_{\tau} / \mu\right)}{\operatorname{det}\left(j \partial_{\tau}+\partial_{\tau}^{2} / \mu\right)}=-\frac{1}{2 i \sin (z / 2)} .
$$

Hence the one-loop factor has the same limit whether $\beta \mu \rightarrow \infty$ or $\beta \mu \rightarrow 0 .{ }^{17}$

\subsubsection{Finite temperature}

We have shown in Lorentzian signature and at zero temperature that integrating out the fermions in the supersymmetric theory with isospin $J$ ( $2 J$ bosonic ground states) yields an effective bosonic theory with isospin $j=J-1 / 2(2 j+1$ bosonic ground states), which is consistent with the equality of $I_{\mathcal{N}=0}(j)$ in (4.53) and $I_{\mathcal{N}=2}(J)$ in (4.58).

The index, however, is computed at finite temperature. The temperature can only enter the effective action through the dimensionless combination $\beta \mu$, and this dependence must disappear in the limit $\mu \rightarrow \infty$. Therefore, the statement of the preceding paragraph must be independent of temperature. Let us show this directly at finite temperature by mimicking the index computation, thereby giving an alternative and cleaner derivation of (4.29).

We first perform a field redefinition $\psi^{\prime}=\psi /\left(1+|\phi|^{2}\right)$ (the associated Jacobian determinant cancels in regularization). Integrating by parts then gives

$$
L_{F, E}=\psi^{\prime \dagger} \mathcal{D} \psi^{\prime}, \quad \mathcal{D} \equiv \frac{\partial_{\tau}+\mathcal{L}_{0, E}}{\mu}+j, \quad \mathcal{L}_{0, E} \equiv \frac{\phi \dot{\phi}^{\dagger}-\phi^{\dagger} \dot{\phi}}{1+|\phi|^{2}}
$$

In Euclidean signature, the eigenfunctions of $\mathcal{D}$ are simple:

$$
f(\tau)=\exp \left[(\lambda-j) \mu \tau-\int^{\tau} d \tau^{\prime} \mathcal{L}_{0, E}\right] .
$$

With periodic (supersymmetric) boundary conditions for the fermions, the eigenvalues are

$$
\lambda_{n}=j+\frac{2 \pi i n+\mathcal{A}}{\beta \mu}, \quad \mathcal{A}=\int_{0}^{\beta} d \tau \mathcal{L}_{0, E}, \quad n \in \mathbb{Z} .
$$

Free-field subtraction then gives

$$
\frac{\operatorname{det}\left(\partial_{\tau} / \mu+j+\mathcal{L}_{0, E} / \mu\right)}{\operatorname{det}\left(\partial_{\tau} / \mu+j\right)}=\prod_{n=-\infty}^{\infty} \frac{j+(2 \pi i n+\mathcal{A}) / \beta \mu}{j+2 \pi i n / \beta \mu}=\frac{e^{-\mathcal{A} / 2}\left(1-e^{\mathcal{A}+\beta \mu j}\right)}{1-e^{\beta \mu j}}
$$

Upon taking $\mu \rightarrow \infty$, this becomes $e^{(j /|j|) \mathcal{A} / 2}$, whose exponent has the correct sign because the Euclidean action appears with a minus sign in the path integral.

Note that while this computation seemingly fixes the sign outright, our regularization crucially assumes a positive sign for $\mu$. Moreover, different regularization schemes lead to

\footnotetext{
${ }^{17}$ To complete the argument, one should check that the path integral measure is invariant under $Q$ (and/or $Q^{\dagger}$ ). While we have assumed that this measure reduces to $D \Delta^{\dagger} D \Delta D \Xi^{\dagger} D \Xi$ for fluctuations $(D$ here should not be confused with a superderivative), the full nonperturbative path integral measure (i.e., the supersymmetrized Fubini-Study measure) must be invariant under both SUSY and global SU(2).
} 
different global anomalies in the effective action [42, 43]. For instance, using Hurwitz zeta function regularization before free-field subtraction would give

$$
\frac{1-e^{\mathcal{A}+\beta \mu j}}{1-e^{\beta \mu j}} \stackrel{\mu \rightarrow \infty}{\longrightarrow} e^{(1+j /|j|) \mathcal{A} / 2} .
$$

These ambiguities can be phrased as a mixed anomaly between the "charge conjugation" symmetry taking $z \rightarrow-z$ and invariance under global gauge transformations $z \rightarrow z+2 \pi n$ for $n \in \mathbb{Z}[43]$ (as we will see shortly, $z$ can be interpreted as a background gauge field). Indeed, in terms of the effective bosonic system, $I_{\mathcal{N}=0}$ in (4.53) is invariant under $z \rightarrow$ $z+2 \pi n$ for integer $j$ but picks up a sign of $(-1)^{n}$ for half-integer $j$. On the other hand, in an alternate regularization where $I_{\mathcal{N}=0} \rightarrow e^{i(j+1 / 2) z} I_{\mathcal{N}=0}, I_{\mathcal{N}=0}$ is no longer even in $z$ but picks up a sign of $(-1)^{n}$ for all $j$. To fix the sign of the shift unambiguously (i.e., such that the effective action computation is consistent with the index), we appeal to canonical quantization. In other words, in the Hamiltonian formalism, we demand that the $\mathrm{SU}(2)$ symmetry be preserved quantum-mechanically.

\subsubsection{Background gauge field}

The quantities (4.41) are useful because the twisted index with vanishing background gauge field is in fact equivalent to the untwisted index with arbitrary constant background gauge field. To see this, set $\mu=\infty$ for simplicity. To restore the background gauge field, we simply take $L_{B} \rightarrow L_{B}+j \mathcal{L}_{A}$, or equivalently

$$
L_{B, E} \rightarrow L_{B, E}-j \mathcal{L}_{A},
$$

with $\mathcal{L}_{A}$ in (4.8) (note that $\mathcal{L}_{A, E}=-\mathcal{L}_{A}$, where the gauge field is always written in Lorentzian conventions). With $A_{i}=0$, the bosonic index $I_{\mathcal{N}=0}$ corresponds to the partition function for $L_{B, E}$ on $S^{1}$ with twisted boundary conditions implemented by the quantum operator $L_{3}$, whose classical expression is given in (C.42). Clearly, $I_{\mathcal{N}=0}$ can also be viewed as a thermal partition function for a deformed Hamiltonian with periodic boundary conditions:

$$
I_{\mathcal{N}=0}=\operatorname{Tr}\left(e^{-\beta H_{z}}\right), \quad H_{z} \equiv H-\frac{i z L_{3}}{\beta}=H+\frac{i j z}{\beta} \frac{1-|\phi|^{2}}{1+|\phi|^{2}} .
$$

This corresponds to a path integral with the modified Lagrangian

$$
L_{B, E}+\frac{i j z}{\beta} \frac{1-|\phi|^{2}}{1+|\phi|^{2}} .
$$

Setting $z=i \beta A_{3}$, we recover precisely $\left.\left(L_{B, E}-j \mathcal{L}_{A}\right)\right|_{A_{1}=A_{2}=0}$, so we deduce from (4.53) that

$$
\int D \phi^{\dagger} D \phi e^{-\left.\int_{0}^{\beta} d \tau\left(L_{B, E}-j \mathcal{L}_{A}\right)\right|_{A_{1}=A_{2}=0}}=\frac{\sinh \left((j+1 / 2) \beta A_{3}\right)}{\sinh \left(\beta A_{3} / 2\right)},
$$

with periodic boundary conditions implicit. But for a constant gauge field, we can always change the basis in group space to set $A_{1}=A_{2}=0$ : under a finite global SU(2) transformation $\phi \rightarrow(a \phi+b) /(-\bar{b} \phi+\bar{a})$, the measure is invariant, the single-derivative Wess-Zumino 
term changes by a total derivative, and the Noether currents (4.16) rotate into each other. Letting $|A|=\sqrt{\sum_{i} A_{i}^{2}}$ denote the norm in group space, we conclude that

$$
\int D \phi^{\dagger} D \phi e^{-\int_{0}^{\beta} d \tau\left(L_{B, E}-j \mathcal{L}_{A}\right)}=\frac{\sinh ((j+1 / 2) \beta|A|)}{\sinh (\beta|A| / 2)} \cdot{ }^{18}
$$

Setting $L_{B, E}^{\mathrm{WZ}} \equiv j \mathcal{L}_{0, E}$ and noting that $\operatorname{Tr}_{j} e^{ \pm \beta A_{i} J_{i}}=\operatorname{Tr}_{j} e^{\beta|A| J_{3}}$, this result can be written more suggestively as

$$
\int D \phi^{\dagger} D \phi \exp \left[-\int_{0}^{\beta} d \tau\left(L_{B, E}^{\mathrm{WZ}}-2 j A_{i} J_{i}\right)\right]=\operatorname{Tr}_{j} e^{-\beta A_{i} J_{i}}
$$

where on the left, the $J_{i}$ are interpreted as classical Noether currents and on the right, they are interpreted as quantum non-commuting matrices (the Hermitian generators of SU(2) in the spin- $j$ representation). Hence the path integral for the 1D quantum mechanics with constant background gauge field computes a Wilson loop of spin $j$ with constant gauge field along the $S^{1}$, i.e., the character of the spin- $j$ representation. This identification holds even for arbitrary background gauge field because one can always choose a time-dependent gauge such that the gauge field is constant along the loop; the only invariant information is the conjugacy class of the holonomy around the loop. Indeed, a Wilson loop can be thought of as a dynamical generalization of a Weyl character.

We can now be even more explicit about the relation between the standard pathordered definition of a Wilson loop and the coadjoint orbit description, with path ordering identified with time ordering in the quantum mechanics on the line and noncommutativity arising as a quantum effect. In this way, we derive Kirillov's character formula from the partition function of the quantum mechanics [44]. Take the gauge field along the $S^{1}$ to be time-dependent and consider the path-ordered exponential

$$
P \equiv P \exp \left[\int_{0}^{T} d t(A+B(t))\right]=\sum_{n=0}^{\infty} \int_{0}^{T} d t_{1} \int_{0}^{t_{1}} d t_{2} \cdots \int_{0}^{t_{n-1}} d t_{n}\left(A+B\left(t_{1}\right)\right) \cdots\left(A+B\left(t_{n}\right)\right)
$$

where $A, B$ are matrices and $A$ is constant. Observe that $P=P^{\prime}$ where

$$
P^{\prime}=\sum_{n=0}^{\infty} \int_{0}^{T} d t_{1} \int_{0}^{t_{1}} d t_{2} \cdots \int_{0}^{t_{n-1}} d t_{n} e^{\left(T-t_{1}\right) A} B\left(t_{1}\right) e^{\left(t_{1}-t_{2}\right) A} B\left(t_{2}\right) \cdots e^{\left(t_{n-1}-t_{n}\right) A} B\left(t_{n}\right) e^{t_{n} A}
$$

because $P$ and $P^{\prime}$ both satisfy the differential equation $f^{\prime}(T)=(A+B(T)) f(T)$ subject to the initial condition $f(0)=1$. Now consider a Euclideanized Wilson loop wrapping the $S^{1}$ and split the gauge field into a fiducial time-independent part and the remainder:

$$
\operatorname{Tr}_{j} P \exp \left[-\int_{0}^{\beta} d \tau\left(A_{i}^{c}+A_{i}^{\tau}\right) J_{i}\right] \equiv \operatorname{Tr}_{j} P \exp \left[\int_{0}^{\beta} d \tau(A+\mathcal{A})\right] \equiv \sum_{n=0}^{\infty} P_{n} .
$$

\footnotetext{
${ }^{18}$ While we inferred this result from the $\mathrm{SU}(2)$ symmetry of the twisted partition function, it can also be seen directly from a semiclassical analysis of the Euclidean Lagrangian. Setting $\mu=\infty$ and imposing periodic boundary conditions, the critical points of $L_{B, E}-j \mathcal{L}_{A}$ occur at the constant values

$$
\phi_{\mathrm{cl}}=\left.\frac{A_{3} \pm|A|}{A_{1}+i A_{2}} \Longrightarrow\left(L_{B, E}-j \mathcal{L}_{A}\right)\right|_{\phi_{\mathrm{cl}}}= \pm j|A| .
$$

Including the one-loop determinants around these classical contributions gives the expected answer.
} 
One can view the terms $P_{n}$ as operator insertions inside

$$
\int D \phi^{\dagger} D \phi \exp \left[-\int_{0}^{\beta} d \tau\left(L_{B, E}^{\mathrm{WZ}}+2 j A\right)\right]=\operatorname{Tr}_{j} e^{\beta A}
$$

(where the implicit $J_{i}$ are classical on the left and quantum on the right) as follows:

$$
\begin{aligned}
P_{n} & =\int_{0}^{\beta} d \tau_{1} \int_{0}^{\tau_{1}} d \tau_{2} \cdots \int_{0}^{\tau_{n-1}} d \tau_{n} \operatorname{Tr}_{j}\left(e^{\left(\beta-\tau_{1}\right) A} \mathcal{A}\left(\tau_{1}\right) e^{\left(\tau_{1}-\tau_{2}\right) A} \mathcal{A}\left(\tau_{2}\right) \cdots e^{\left(\tau_{n-1}-\tau_{n}\right) A} \mathcal{A}\left(\tau_{n}\right) e^{\tau_{n} A}\right) \\
& =\int_{0}^{\beta} d \tau_{1} \int_{0}^{\tau_{1}} d \tau_{2} \cdots \int_{0}^{\tau_{n-1}} d \tau_{n} \int D \phi^{\dagger} D \phi\left[\prod_{i=1}^{n}-2 j \mathcal{A}\left(\tau_{i}\right)\right] \exp \left[-\int_{0}^{\beta} d \tau\left(L_{B, E}^{\mathrm{WZ}}+2 j A\right)\right] \\
& =\frac{1}{n !} \int D \phi^{\dagger} D \phi\left[\prod_{i=1}^{n}-2 j \int_{0}^{\beta} d \tau_{i} \mathcal{A}\left(\tau_{i}\right)\right] \exp \left[-\int_{0}^{\beta} d \tau\left(L_{B, E}^{\mathrm{WZ}}+2 j A\right)\right] .
\end{aligned}
$$

In the last step, we have used that the $\mathcal{A}\left(\tau_{i}\right)$ are classical quantities inside the path integral. Hence the sum exponentiates to

$$
\operatorname{Tr}_{j} P \exp \left[-\int_{0}^{\beta} d \tau\left(A_{i}^{c}+A_{i}^{\tau}\right) J_{i}\right]=\int D \phi^{\dagger} D \phi \exp \left[-\int_{0}^{\beta} d \tau\left(L_{B, E}^{\mathrm{WZ}}-2 j\left(A_{i}^{c}+A_{i}^{\tau}\right) J_{i}\right)\right]
$$

where again, the $J_{i}$ on the left and right have different meanings.

The above arguments can be carried over wholesale to the supersymmetric index $I_{\mathcal{N}=2}$, since the $\left(L_{f}\right)_{i}$ rotate into each other under global SU(2). The fermions modify the representation in which the trace is taken, and (as we will see) the fact that a particular linear combination of the bulk gauge field and the auxiliary scalar $\sigma$ appears in the quantum mechanics is reflected in the appearance of these fields in the supersymmetric path-ordered expression.

\section{Coupling to the bulk}

We now take a top-down approach to the quantum mechanics on the line by restricting the $3 \mathrm{D} \mathcal{N}=2$ multiplets to $1 \mathrm{D} \mathcal{N}=2$ multiplets closed under SUSY transformations that generate translations along the line, which we take to extend along the 0 direction in $\mathbb{R}^{1,2}$ (as in the previous section, aside from section 4.3, we work in Lorentzian signature). We thus identify the components of the $1 \mathrm{D}$ vector multiplet with restrictions of the bulk fields; in principle, the 1D chiral multiplet $\Phi$ of the previous section descends from bulk super gauge transformations.

Our conventions for SUSY in $\mathbb{R}^{1,2}$ are given in appendix A.3. The linear combination of supercharges that generates translations along the line is $\Omega \equiv\left(Q_{1}+i Q_{2}\right) / \sqrt{2}$ (any choice $\Omega=c_{1} Q_{1}+c_{2} Q_{2}$ with $\left|c_{1}\right|^{2}=\left|c_{2}\right|^{2}=1 / 2$ and $c_{1} c_{2}^{*}$ purely imaginary would suffice), which satisfies $\left\{\Omega, \Omega^{\dagger}\right\}=-2 P_{0}=2 H$ for vanishing central charge. Therefore, to restrict to the line, we choose the infinitesimal spinor parameter $\xi$ such that

$$
\xi Q=\xi_{1} Q_{2}-\xi_{2} Q_{1}=\omega \Omega \Longrightarrow\left(\xi_{1}, \xi_{2}\right)=\frac{1}{\sqrt{2}}(i \omega,-\omega)
$$


where $\omega$ is some fiducial Grassmann parameter (note that $\xi Q$ has suppressed spinor indices, while $\omega \Omega$ does not). In terms of the linear representations of the supercharges on $3 \mathrm{D}$ and $1 \mathrm{D} \mathcal{N}=2$ superspace ((A.19) and (A.4), respectively), we compute that for superfields whose only spacetime dependence is on the 0 direction, $1 \mathrm{D} \mathcal{N}=2$ SUSY transformations are implemented by $\xi \mathcal{Q}-\bar{\xi} \overline{\mathcal{Q}}=\omega \hat{Q}+\bar{\omega} \hat{Q}^{\dagger}$ with $\theta=\frac{1}{\sqrt{2}}\left(\theta^{1}-i \theta^{2}\right)$ and $\partial_{\theta}=\frac{1}{\sqrt{2}}\left(\partial_{\theta^{1}}+i \partial_{\theta^{2}}\right)$.

\subsection{Linearly realized SUSY on the line}

With all auxiliary fields necessary to realize SUSY transformations linearly, a $3 \mathrm{D} \mathcal{N}=2$ vector multiplet $\left(V=V^{\dagger}\right)$ takes the form

$$
\begin{aligned}
V= & C+\theta \chi-\bar{\theta} \bar{\chi}+\frac{1}{2} \theta^{2}(M+i N)-\frac{1}{2} \bar{\theta}^{2}(M-i N)-i \theta \bar{\theta} \sigma-\theta \gamma^{\mu} \bar{\theta} A_{\mu} \\
& +i \theta^{2} \bar{\theta}\left(\bar{\lambda}-\frac{1}{2} \gamma^{\mu} \partial_{\mu} \chi\right)-i \bar{\theta}^{2} \theta\left(\lambda-\frac{1}{2} \gamma^{\mu} \partial_{\mu} \bar{\chi}\right)+\frac{1}{2} \theta^{2} \bar{\theta}^{2}\left(D-\frac{1}{2} \partial^{2} C\right)
\end{aligned}
$$

where $V=V^{a} T^{a}$, etc., and all bosonic components are real. A 3D $\mathcal{N}=2$ chiral multiplet $\left(\bar{D}_{\alpha} \Phi=0\right)$ takes the form

$$
\Phi=A-i \theta \gamma^{\mu} \bar{\theta} \partial_{\mu} A-\frac{1}{4} \theta^{2} \bar{\theta}^{2} \partial^{2} A+\sqrt{2} \theta \psi-\frac{i}{\sqrt{2}} \theta^{2} \bar{\theta} \gamma^{\mu} \partial_{\mu} \psi+\theta^{2} F
$$

where the scalar components are complex. Bulk (3D) SUSY acts on the vector and chiral multiplets as in (A.21) and (A.22). For $f$ any complex 3D fermion, it is convenient to set

$$
f^{\prime} \equiv \frac{f_{1}+i f_{2}}{\sqrt{2}}, \quad f^{\prime \prime} \equiv \frac{f_{1}-i f_{2}}{\sqrt{2}}
$$

We find that the $3 \mathrm{D} \mathcal{N}=2$ vector multiplet restricts to the following $1 \mathrm{D} \mathcal{N}=2$ multiplets:

- a $1 \mathrm{D}$ vector $\left\{-C, \chi^{\prime}, \sigma+A_{0}\right\}$,

- a $1 \mathrm{D}$ chiral $\left\{(N+i M) / 2, \lambda^{\prime}-i \partial_{0} \bar{\chi}^{\prime \prime}\right\}$ (and its conjugate antichiral),

- and a $1 \mathrm{D}$ chiral $\left\{\left(i D-\partial_{0} \sigma\right) / 2, \partial_{0} \bar{\lambda}^{\prime \prime}\right\}$ (and its conjugate antichiral).

We find that the $3 \mathrm{D} \mathcal{N}=2$ chiral multiplet restricts to the following $1 \mathrm{D} \mathcal{N}=2$ multiplets:

- a $1 \mathrm{D}$ chiral $\left\{A,-\sqrt{2} \psi^{\prime}\right\}$

- and a 1D antichiral $\left\{F,-\sqrt{2} \partial_{0} \psi^{\prime \prime}\right\}$.

The above $1 \mathrm{D} \mathcal{N}=2$ multiplets transform according to (A.10) and (A.12) with $\epsilon=\omega$. Note that $\chi, \lambda, \psi$ in $3 \mathrm{D}$ each restrict to two independent complex fermions in $1 \mathrm{D}$.

\subsection{Nonlinearly realized SUSY on the line}

The most direct way to see how $\Phi$ in the coadjoint orbit Lagrangian arises from bulk super gauge transformations would be to perform the supersymmetric analogue of the derivation of section 3.2 by cutting out a tubular neighborhood of the line and examining the effect of a bulk super gauge transformation on the resulting boundary (an action is induced on 
the line after integrating over all such transformations and taking the radius to zero). For this derivation, it would not suffice to work in Wess-Zumino gauge. ${ }^{19}$ Therefore, we do not presuppose a gauge. In superspace, the $3 \mathrm{D} \mathcal{N}=2$ Chern-Simons Lagrangian

$$
\mathcal{L}_{\mathrm{CS}}=\frac{k}{4 \pi i} \int d^{4} \theta \int_{0}^{1} d t \operatorname{Tr}\left[V \bar{D}_{\alpha}\left(e^{-2 t V} D^{\alpha} e^{2 t V}\right)\right]
$$

is invariant under (linearly realized) SUSY and reduces to (2.2) in Wess-Zumino gauge. To see the effect of a super gauge transformation (following [45]), consider more generally

$$
\mathcal{L}_{\mathrm{CS}}=\frac{k}{8 \pi i} \int d^{4} \theta \int_{0}^{1} d t \ell_{\mathrm{CS}}, \quad \ell_{\mathrm{CS}}=\operatorname{Tr}\left[\left(e^{-2 V(t)} \partial_{t} e^{2 V(t)}\right) \bar{D}_{\alpha}\left(e^{-2 V(t)} D^{\alpha} e^{2 V(t)}\right)\right]
$$

with boundary conditions $V(0)=0$ and $V(1)=V$. Under a super gauge transformation $e^{2 V(t)} \rightarrow e^{\bar{\Phi}(t)} e^{2 V(t)} e^{\Phi(t)}$, we have $\ell_{\mathrm{CS}} \rightarrow \ell_{\mathrm{CS}}+\delta^{\prime} \ell_{\mathrm{CS}}$ where

$$
\delta^{\prime} \ell_{\mathrm{CS}}=\operatorname{Tr}\left[D^{\alpha}\left(e^{-\bar{\Phi}(t)} \partial_{t} e^{\bar{\Phi}(t)} e^{2 V(t)} \bar{D}_{\alpha} e^{-2 V(t)}\right)+\bar{D}_{\alpha}\left(\partial_{t} e^{\Phi(t)} e^{-\Phi(t)} e^{-2 V(t)} D^{\alpha} e^{2 V(t)}\right)\right] .
$$

Obtaining an explicit expression for this total derivative (in particular, for $\Phi(t)$ when $V(t)=t V)$ is prohibitively complicated. Thus, rather than imitating the derivation of [37], we will arrive at a bulk interpretation of the quantum-mechanical variables $\phi, \psi$ in WessZumino gauge, which partially fixes "super gauge" while retaining the freedom to perform ordinary gauge transformations. To this end, it is useful to work in terms of the correponding nonlinearly realized supersymmetry (SUSY') transformations.

In Wess-Zumino gauge, a $3 \mathrm{D} \mathcal{N}=2$ vector multiplet takes the form

$$
\left.V\right|_{\mathrm{WZ}}=-i \theta \bar{\theta} \sigma-\theta \gamma^{\mu} \bar{\theta} A_{\mu}+i \theta^{2} \bar{\theta} \bar{\lambda}-i \bar{\theta}^{2} \theta \lambda+\frac{1}{2} \theta^{2} \bar{\theta}^{2} D
$$

Bulk (3D) SUSY' acts on the vector multiplet as

$$
\begin{aligned}
\delta^{\prime} \sigma & =-(\xi \bar{\lambda}-\bar{\xi} \lambda), \\
\delta^{\prime} A_{\mu} & =i\left(\xi \gamma_{\mu} \bar{\lambda}+\bar{\xi} \gamma_{\mu} \lambda\right), \\
\delta^{\prime} \lambda & =-i \xi D-i \gamma^{\mu} \xi D_{\mu} \sigma-\frac{1}{2} \epsilon^{\mu \nu \rho} \gamma_{\rho} \xi F_{\mu \nu}, \\
\delta^{\prime} \bar{\lambda} & =i \bar{\xi} D+i \gamma^{\mu} \bar{\xi} D_{\mu} \sigma-\frac{1}{2} \epsilon^{\mu \nu \rho} \gamma_{\rho} \bar{\xi} F_{\mu \nu}, \\
\delta^{\prime} D & =-\left(\xi \gamma^{\mu} D_{\mu} \bar{\lambda}-\bar{\xi} \gamma^{\mu} D_{\mu} \lambda\right)+[\xi \bar{\lambda}+\bar{\xi} \lambda, \sigma]
\end{aligned}
$$

where $D_{\mu}(\cdot)=\partial_{\mu}(\cdot)-i\left[A_{\mu},(\cdot)\right]$ and $F_{\mu \nu}=\partial_{\mu} A_{\nu}-\partial_{\nu} A_{\mu}-i\left[A_{\mu}, A_{\nu}\right]$. Bulk (3D) SUSY' acts on a fundamental chiral multiplet as

$$
\begin{aligned}
& \delta^{\prime} A=-\sqrt{2} \xi \psi \\
& \delta^{\prime} \psi=-\sqrt{2} \xi F+i \sqrt{2} \gamma^{\mu} \bar{\xi} D_{\mu} A+i \sqrt{2} \bar{\xi} \sigma A \\
& \delta^{\prime} F=i \sqrt{2} \bar{\xi} \gamma^{\mu} D_{\mu} \psi-i \sqrt{2} \sigma \bar{\xi} \psi-2 i \bar{\xi} \bar{\lambda} A
\end{aligned}
$$

\footnotetext{
${ }^{19}$ The conditions on the chiral superfield transformation parameter $\Lambda$ to preserve Wess-Zumino gauge are that $A=-A^{*}, \psi=0$, and $F=0$, in which case the super gauge transformation $e^{2 V} \rightarrow e^{\bar{\Lambda}} e^{2 V} e^{\Lambda}$ reduces to an ordinary gauge transformation with parameter $-A: V \rightarrow V-i \theta \gamma^{\mu} \bar{\theta} \partial_{\mu} A+[V, A]$. These conditions preclude the possibility of inducing fermions on the line.
} 
where $D_{\mu}(\cdot)=\partial_{\mu}(\cdot)-i A_{\mu}(\cdot)$. SUSY' transformations close off shell into the algebra

$$
\left[\delta_{\zeta}^{\prime}, \delta_{\xi}^{\prime}\right](\cdot)=-2 i\left(\xi \gamma^{\mu} \bar{\zeta}+\bar{\xi} \gamma^{\mu} \zeta\right) D_{\mu}(\cdot)-2 i(\xi \bar{\zeta}-\bar{\xi} \zeta) \sigma \cdot(\cdot)
$$

on gauge-covariant fields where, e.g., $\sigma \cdot(\cdot) \equiv[\sigma,(\cdot)]$ for $\sigma, F_{\mu \nu}, \lambda, \bar{\lambda}, D$ and $\sigma \cdot(\cdot) \equiv \sigma(\cdot)$ for $A, \psi, F$. The above transformation laws and commutators can be obtained by dimensional reduction from $4 \mathrm{D}$ (set $\partial_{3}=0$ ).

The 3D SUSY' transformations restrict to the line as follows. We again use the notation (5.4). For the vector multiplet, defining the SUSY'-covariant derivative $D_{0}^{\prime}(\cdot) \equiv$ $D_{0}(\cdot)-i[\sigma,(\cdot)]=\partial_{0}(\cdot)-i\left[\sigma+A_{0},(\cdot)\right]$, which satisfies $\delta^{\prime} D_{0}^{\prime}(\cdot)=D_{0}^{\prime} \delta^{\prime}(\cdot)$ and $D_{0}^{\prime} \sigma=D_{0} \sigma$, we obtain the following (rather degenerate) restricted multiplets in 1D:

- a $1 \mathrm{D}$ vector $\left\{0,0, \sigma+A_{0}\right\}$,

- a $1 \mathrm{D}$ adjoint chiral $\left\{0, \lambda^{\prime}\right\}$ (and its complex conjugate),

- and a $1 \mathrm{D}$ adjoint chiral $\left\{\left(i D-D_{0}^{\prime} \sigma\right) / 2, D_{0}^{\prime} \bar{\lambda}^{\prime \prime}\right\}$ (and its complex conjugate).

For a fundamental chiral multiplet, defining the SUSY'-covariant derivative $D_{0}^{\prime}(\cdot) \equiv D_{0}(\cdot)-$ $i \sigma(\cdot)=\partial_{0}(\cdot)-i\left(\sigma+A_{0}\right)(\cdot)$, which satisfies $\delta^{\prime} D_{0}^{\prime}(\cdot)=D_{0}^{\prime} \delta^{\prime}(\cdot)$, we obtain a single restricted multiplet in $1 \mathrm{D}$, namely

- a $1 \mathrm{D}$ fundamental chiral $\left\{A,-\sqrt{2} \psi^{\prime}\right\}$,

whose scalar component is associated with bulk gauge transformations. All of the above $1 \mathrm{D} \mathcal{N}=2$ chiral multiplets transform according to (A.14) with $\epsilon=\omega$ and $D_{0} \rightarrow D_{0}^{\prime}$. Note that the putative 1D fundamental antichiral $\left\{F,-\sqrt{2} D_{0}^{\prime} \psi^{\prime \prime}\right\}$ transforms according to

$$
\begin{aligned}
\delta^{\prime} F & =-\bar{\omega}\left(-\sqrt{2} D_{0}^{\prime} \psi^{\prime \prime}\right)-2 i A \bar{\omega} \bar{\lambda}^{\prime}, \\
\delta^{\prime}\left(-\sqrt{2} D_{0}^{\prime} \psi^{\prime \prime}\right) & =2 i \omega D_{0}^{\prime} F,
\end{aligned}
$$

which is incompatible with 1D SUSY'. On a 1D chiral multiplet, the 1D SUSY' algebra is realized as

$$
\left[\delta_{\eta}^{\prime}, \delta_{\epsilon}^{\prime}\right](\cdot)=-2 i\left(\epsilon \eta^{\dagger}+\epsilon^{\dagger} \eta\right) D_{0}^{\prime}(\cdot)
$$

for $(\cdot)=\phi, \psi$, while $\delta^{\prime}$ acts trivially on a $1 \mathrm{D}$ vector multiplet in Wess-Zumino gauge.

One would expect to write a coupled 3D-1D action

$$
S_{3 \mathrm{D}-1 \mathrm{D}}=\int d^{3} x \mathcal{L}_{\mathrm{CS}}+j \int d t \tilde{\mathcal{L}}
$$

that is both supersymmetric and gauge-invariant (under SUSY' and ordinary gauge transformations), with the transformation of the 1D action compensating for any boundary terms induced along the line in the transformation of the 3D action. However, in WessZumino gauge, $\mathcal{L}_{\mathrm{CS}}$ in (2.2) has the following SUSY' variation:

$$
\delta^{\prime} \mathcal{L}_{\mathrm{CS}}=\frac{k}{4 \pi} \partial_{\mu} \operatorname{Tr}\left[i \epsilon^{\mu \nu \rho}\left(\xi \gamma_{\nu} \bar{\lambda}+\bar{\xi} \gamma_{\nu} \lambda\right) A_{\rho}+2\left(\xi \gamma^{\mu} \bar{\lambda}-\bar{\xi} \gamma^{\mu} \lambda\right) \sigma\right]
$$

This induces a boundary term along the line only if the fields are singular as the inverse of the radial distance to the line. Since they are not, it suffices to show that the $1 \mathrm{D}$ action is itself invariant under appropriately defined 1D SUSY' transformations. 


\subsection{Nonlinearly realized SUSY in the sigma model}

To carry out this last step, we specialize to $\mathrm{SU}(2)$. For the vector multiplet, the bulk and line variables are identified as $a_{i}=-C_{i}, \psi_{i}=\chi_{i}^{\prime}, A_{i}=\sigma_{i}+\left(A_{0}\right)_{i}$. The quantum mechanics $\left.\tilde{\mathcal{L}}\right|_{\text {WZ }}=\tilde{\mathcal{L}}_{0}+\mathcal{L}_{A}$ is invariant under the 1D SUSY' transformations

$$
\begin{aligned}
\delta^{\prime} \phi & =\epsilon \psi, \\
\delta^{\prime} \psi & =-2 i \epsilon^{\dagger}\left(\dot{\phi}-\frac{i}{2} A_{1}\left(1-\phi^{2}\right)-\frac{1}{2} A_{2}\left(1+\phi^{2}\right)-i A_{3} \phi\right),
\end{aligned}
$$

which satisfy the algebra

$$
\begin{aligned}
{\left[\delta_{\eta}^{\prime}, \delta_{\epsilon}^{\prime}\right] \phi } & =-2 i\left(\epsilon \eta^{\dagger}+\epsilon^{\dagger} \eta\right)\left(\dot{\phi}-\frac{i}{2} A_{-}+\frac{i}{2} A_{+} \phi^{2}-i A_{3} \phi\right), \\
{\left[\delta_{\eta}^{\prime}, \delta_{\epsilon}^{\prime}\right] \psi } & =-2 i\left(\epsilon \eta^{\dagger}+\epsilon^{\dagger} \eta\right)\left(\dot{\psi}+i\left(A_{+} \phi-A_{3}\right) \psi\right), \\
{\left[\delta_{\eta}^{\prime}, \delta_{\epsilon}^{\prime}\right] \psi^{\dagger} } & =-2 i\left(\epsilon \eta^{\dagger}+\epsilon^{\dagger} \eta\right)\left(\dot{\psi}^{\dagger}-i\left(A_{-} \phi^{\dagger}-A_{3}\right) \psi^{\dagger}\right) .
\end{aligned}
$$

The adjoint action of $\mathrm{SU}(2)$ on its Lie algebra induces an action on $S^{2}$, which explains the appearance of the $\mathrm{SU}(2)$ Killing vectors in $\delta^{\prime} \psi$. Explicitly, at the level of scalar components, the map between the adjoint (gauge parameter) chiral superfield $S=s+\theta f-i \theta \theta^{\dagger} \dot{s}=$ $S^{a} \sigma^{a} / 2$ and the (scalar) $\mathrm{SU}(2) / \mathrm{U}(1)$ coset chiral superfield $\Phi=\phi+\theta \psi-i \theta \theta^{\dagger} \dot{\phi}$ is

$$
\frac{1}{|s|}\left(\begin{array}{c}
s^{1} \\
-s^{2} \\
s^{3}
\end{array}\right) \leftrightarrow\left(\begin{array}{c}
\sin \theta \cos \varphi \\
\sin \theta \sin \varphi \\
\cos \theta
\end{array}\right) \leftrightarrow \phi=\frac{s^{1}-i s^{2}}{|s|-s^{3}}
$$

by stereographic projection (note that this only makes sense for $s$ real). In terms of angles,

$$
e^{i \varphi}=\frac{s^{1}-i s^{2}}{\sqrt{|s|^{2}-\left(s^{3}\right)^{2}}}, \quad \tan (\theta / 2)=\sqrt{\frac{|s|-s^{3}}{|s|+s^{3}}} .
$$

Keep in mind that to translate between the adjoint action and linear fractional transformations, one must flip the sign of the second Killing vector: that is, one must identify $\vec{\sigma} / 2$ with $\left(\vec{e}_{1},-\vec{e}_{2}, \vec{e}_{3}\right)$. The action of $\mathrm{SU}(2)$ is as expected: writing $\epsilon=\epsilon^{a} \sigma^{a} / 2$ and $s=s^{a} \sigma^{a} / 2$, we have with $\epsilon^{i}$ infinitesimal that

$$
g=1+i \epsilon \Longrightarrow g s g^{-1}=s+i[\epsilon, s] \Longrightarrow \delta_{\mathrm{SU}(2)} s^{i}=\epsilon^{i j k} s^{j} \epsilon^{k} .
$$

Under the given map (5.17), this is equivalent to $\delta_{\mathrm{SU}(2)} \phi=\epsilon_{i} x_{i}$. Now we check that SUSY' acts correctly. Naïvely, we have for the components of $S$ that (with $A=A^{a} \sigma^{a} / 2$ )

$$
\begin{aligned}
\delta^{\prime} s & =\epsilon f, \\
\delta^{\prime} f & =-2 i \epsilon^{\dagger}(\dot{s}-i[A, s]),
\end{aligned}
$$

but to make sense of SUSY' transformations for real $s$, we must take $f$ real and $\epsilon$ purely imaginary (though $S$ itself is not real):

$$
\begin{aligned}
& \delta^{\prime} s=i \epsilon f, \\
& \delta^{\prime} f=-2 \epsilon(\dot{s}-i[A, s]),
\end{aligned}
$$


where $\epsilon, f$ are real Grassmann variables. In terms of chiral superfields, the desired map is

$$
\Phi=\frac{S^{1}-i S^{2}}{|S|-S^{3}}=\phi+\theta \psi-i \theta \theta^{\dagger} \dot{\phi}
$$

Upon substituting for $\delta^{\prime} s^{a}$ and $\delta^{\prime} f^{a}$, the $\delta^{\prime}$ variations of $\phi=\phi\left(s^{a}, f^{a}\right)$ and $\psi=\psi\left(s^{a}, f^{a}\right)$ are

$$
\begin{aligned}
& \delta^{\prime} \phi=i \epsilon \psi \\
& \delta^{\prime} \psi=-2 \epsilon\left(\dot{\phi}-\frac{i}{2} A^{1}\left(1-\phi^{2}\right)-\frac{1}{2} A^{2}\left(1+\phi^{2}\right)-i A^{3} \phi\right),
\end{aligned}
$$

as expected (for our choice of $\epsilon$ ). It would be interesting to clarify the interpretation of the coadjoint orbit theory as resulting from promoting the gauge transformation parameter in the group element $g$ to a chiral superfield $\left(g=e^{i S^{a} \sigma^{a} / 2}\right)$.

\section{Localization in $3 \mathrm{D}$}

\subsection{Overview}

We now examine how the understanding achieved for a straight line in $\mathbb{R}^{1,2}$ can be extended to certain compact Euclidean spaces.

First, assuming the existence of conformal Killing spinors, we construct in appendix A.3 the curved-space SUSY' transformations (A.37) and (A.39), in which the eight independent superconformal symmetries associated to $\xi, \tilde{\xi}$ generate the $3 \mathrm{D} \mathcal{N}=2$ superconformal algebra $\mathfrak{o s p}(2 \mid 2,2)$. These transformations turn out to be a special case of a more general set of transformations derived from the "new minimal" supergravity background (see [46], which we follow closely in this section). Of course, not all of the backgrounds that we are interested in are conformally flat: having derived the SUSY' transformations with conformal Killing spinor parameters, we restrict to those spinors that generate a suitable non-conformal subalgebra; the resulting transformations, for suitably generalized Killing spinors, pertain to all of the backgrounds that we consider. The results coincide with the supergravity background perspective that we describe in the next subsection.

Having placed the theory supersymmetrically on a curved space, the next question is that of computability. The fact that the Chern-Simons partition function on Seifert manifolds can be written as a matrix model is well-known from [32, 47] (see [33] for a review), and has been discussed in the framework of nonabelian localization in $[13,14]$. By now, the computation of observables in $\mathcal{N}=2$ Chern-Simons theory via supersymmetric localization [48] is also a well-established technique. The original approach of [15] applies to SCFTs (of which pure $\mathcal{N}=2$ Chern-Simons theory is an example [49]), but it can be generalized to non-conformal theories with a $\mathrm{U}(1)_{R}$ symmetry $[50,51]$.

Passing to compact Euclidean three-manifolds allows us to compute correlation functions of nontrivially linked, mutually half-BPS Wilson loops. On $S^{3}$, for example, we can access links whose components are fibers of the same Hopf fibration and are therefore unknots with mutual linking number one (the simplest example is the Hopf link). One can also squash the $S^{3}$ to obtain the Berger sphere with $\mathrm{SU}(2) \times \mathrm{U}(1)$ isometry (where the 
Killing vector has closed orbits and points along the Seifert fiber) $[52,53]$ or the ellipsoid with $\mathrm{U}(1) \times \mathrm{U}(1)$ isometry (where the Killing vector does not, generically, point along the fiber) [24]; on the latter background, one can compute expectation values of nontrivial torus knots $[54,55]$. One can also consider lens spaces [56] and more general Seifert manifolds $[2,26,36,57]$. With appropriate boundary conditions, localizing on a solid torus $D^{2} \times S^{1}[58,59]$ makes contact with supersymmetric analogues of the gluing and Heegaard decompositions usually encountered in the context of Chern-Simons theory [60-63]. We examine the quantum mechanics on Wilson loops in these general backgrounds.

\subsection{Supergravity background}

In the $3 \mathrm{D} \mathcal{N}=2$ context, the background supergravity formalism of [64] allows for the construction of a scalar supercharge by partially topologically twisting the $\mathrm{U}(1)_{R}$ symmetry of the $\mathcal{N}=2$ algebra, when $M^{3}$ admits a transversely holomorphic foliation (THF) [24, 46]. This construction subsumes both the round sphere and squashed sphere backgrounds. The relevant supergravity theory is "new minimal" supergravity, defined as the off-shell formulation of $3 \mathrm{D}$ supergravity that couples to the $\mathcal{R}$-multiplet of a $3 \mathrm{D} \mathcal{N}=2$ quantum field theory with a $\mathrm{U}(1)_{R}$ symmetry. For the supersymmetry algebra and multiplets resulting from the rigid limit of new minimal supergravity, see section 6 of [24]. The bosonic fields in new minimal supergravity are the metric $g_{\mu \nu}$, the $R$-symmetry gauge field $A_{\mu}^{(R)}$, a two-form gauge field $B_{\mu \nu}$, and the central charge symmetry gauge field $C_{\mu}$. It is convenient to let $H$ and $V_{\mu}$ denote the Hodge duals of the field strengths of $B_{\mu \nu}$ and $C_{\mu}$, respectively.

For 3D $\mathcal{N}=2$ theories with a $\mathrm{U}(1)_{R}$ symmetry, [24] classifies the backgrounds that preserve some supersymmetry. In particular, to preserve two supercharges of opposite $R$ charge, the three-manifold $M^{3}$ must admit a nowhere vanishing Killing vector $K^{\mu}$. If $K^{\mu}$ is real, then $M^{3}$ is necessarily an orientable Seifert manifold. An example with $K^{\mu}$ complex is the $S^{2} \times S^{1}$ background of [65] relevant to computing the superconformal index (as opposed to the topologically twisted index of [34]). We focus on the case of a real, nowhere vanishing Killing vector $K^{\mu}$, but we do not restrict the orbit to be a Seifert fiber. Under these assumptions, it suffices to consider backgrounds with $V_{\mu}=0$, so that the conditions for the existence of a rigid supersymmetry are

$$
\left(\nabla_{\mu}-i A_{\mu}^{(R)}\right) \xi=-\frac{1}{2} H \gamma_{\mu} \xi, \quad\left(\nabla_{\mu}+i A_{\mu}^{(R)}\right) \tilde{\xi}=-\frac{1}{2} H \gamma_{\mu} \tilde{\xi}
$$

These are the generalized Killing spinor equations, under which $\xi$ and $\tilde{\xi}$ have $R$-charges \pm 1 , respectively. The corresponding SUSY' transformations with $V_{\mu}=0$ [46] are

$$
\begin{aligned}
\delta^{\prime} \sigma & =-(\xi \tilde{\lambda}-\tilde{\xi} \lambda), \\
\delta^{\prime} A_{\mu} & =i\left(\xi \gamma_{\mu} \tilde{\lambda}+\tilde{\xi} \gamma_{\mu} \lambda\right), \\
\delta^{\prime} \lambda & =-i \xi(D-\sigma H)-i \gamma^{\mu} \xi D_{\mu} \sigma-\frac{i}{2} \sqrt{g}^{-1} \epsilon^{\mu \nu \rho} \gamma_{\rho} \xi F_{\mu \nu}, \\
\delta^{\prime} \tilde{\lambda} & =i \tilde{\xi}(D-\sigma H)+i \gamma^{\mu} \tilde{\xi} D_{\mu} \sigma-\frac{i}{2} \sqrt{g}^{-1} \epsilon^{\mu \nu \rho} \gamma_{\rho} \tilde{\xi} F_{\mu \nu}, \\
\delta^{\prime} D & =-D_{\mu}\left(\xi \gamma^{\mu} \tilde{\lambda}-\tilde{\xi} \gamma^{\mu} \lambda\right)+[\xi \tilde{\lambda}+\tilde{\xi} \lambda, \sigma]+H(\xi \tilde{\lambda}-\tilde{\xi} \lambda)
\end{aligned}
$$


for the vector multiplet and

$$
\begin{aligned}
& \delta^{\prime} A=-\sqrt{2} \xi \psi \\
& \delta^{\prime} \psi=-\sqrt{2} \xi F+i \sqrt{2} \gamma^{\mu} \tilde{\xi} D_{\mu} A+i \sqrt{2} \tilde{\xi} \sigma A-i \sqrt{2} \Delta H \tilde{\xi} A, \\
& \delta^{\prime} F=i \sqrt{2} D_{\mu}\left(\tilde{\xi} \gamma^{\mu} \psi\right)-i \sqrt{2} \sigma \tilde{\xi} \psi-2 i \tilde{\xi} \tilde{\lambda} A+i \sqrt{2}(\Delta-2) H \tilde{\xi} \psi
\end{aligned}
$$

for a fundamental chiral multiplet of dimension $\Delta$. The covariant derivative is now

$$
D_{\mu}=\nabla_{\mu}-i A_{\mu}-i r A_{\mu}^{(R)}
$$

where $r$ is the $R$-charge of the field on which it acts. The transformations (6.2) and (6.3) furnish a representation of the algebra $\mathfrak{s u}(1 \mid 1)$. Taking into account the generalized Killing spinor equations (6.1) and replacing

$$
\nabla_{\mu} \xi \rightarrow D_{\mu} \xi=\left(\nabla_{\mu}-i A_{\mu}^{(R)}\right) \xi, \quad \nabla_{\mu} \tilde{\xi} \rightarrow D_{\mu} \tilde{\xi}=\left(\nabla_{\mu}+i A_{\mu}^{(R)}\right) \tilde{\xi}
$$

in the curved-space SUSY' transformations (A.37) and (A.39) results in precisely the $V_{\mu}=0$ SUSY' transformations above. The latter transformations satisfy the same algebra as (A.37) and (A.39), given in appendix A.3, but with parameters

$$
U^{\mu}=2 i \xi \gamma^{\mu} \tilde{\xi}, \quad \epsilon_{\mu \nu}=2 H \sqrt{g} \epsilon_{\mu \nu \rho} \xi \gamma^{\rho} \tilde{\xi}, \quad \rho=0, \quad \alpha=2 i H \xi \tilde{\xi}
$$

and $D_{\mu}$ as in (6.4). We also have at our disposal

$$
\begin{aligned}
& \delta_{\xi}^{\prime} \delta_{\tilde{\xi}}^{\prime} \operatorname{Tr}\left(\frac{1}{2} \lambda \tilde{\lambda}-i D \sigma\right)=\xi \tilde{\xi} \mathcal{L}_{\mathrm{YM}} \\
& \mathcal{L}_{\mathrm{YM}}=\operatorname{Tr}\left[\frac{1}{4} F_{\mu \nu} F^{\mu \nu}+\frac{1}{2} D_{\mu} \sigma D^{\mu} \sigma-\frac{1}{2}(D-\sigma H)^{2}-i \tilde{\lambda} \gamma^{\mu} D_{\mu} \lambda+i \tilde{\lambda}[\sigma, \lambda]+\frac{i}{2} H \lambda \tilde{\lambda}\right]
\end{aligned}
$$

as a convenient localizing term, where we have omitted the Yang-Mills coupling.

If the generalized Killing spinor equations have at least one solution, then $M^{3}$ admits a THF. The existence of solutions to both equations implies that $K^{\mu} \equiv \xi \gamma^{\mu} \tilde{\xi}$ is a nowhere vanishing Killing vector. Assuming that $K^{\mu}$ is real, we can find local ("adapted") coordinates $(\tilde{\psi}, z, \bar{z})$ such that $K=\partial_{\tilde{\psi}}$ and

$$
d s^{2}=(d \tilde{\psi}+a(z, \bar{z}) d z+\bar{a}(z, \bar{z}) d \bar{z})^{2}+c(z, \bar{z})^{2} d z d \bar{z}
$$

where $a$ is complex and $c$ is real (following [46], we have normalized the metric such that $|K|^{2}=1$, which does not affect results for supersymmetric observables [25]; see also [66]). Coordinate patches are related by transformations of the form $\tilde{\psi}^{\prime}=\tilde{\psi}+\alpha(z, \bar{z}), z^{\prime}=\beta(z)$, $\bar{z}^{\prime}=\bar{\beta}(\bar{z})$ with $\alpha$ real and $\beta$ holomorphic. We choose the vielbein

$$
e^{1}=\frac{1}{2} c(z, \bar{z})(d z+d \bar{z}), \quad e^{2}=\frac{i}{2} c(z, \bar{z})(d z-d \bar{z}), \quad e^{3}=d \tilde{\psi}+a(z, \bar{z}) d z+\bar{a}(z, \bar{z}) d \bar{z},
$$

for which the corresponding spin connection (determined from $d e^{a}+\omega^{a}{ }_{b} \wedge e^{b}=0$ ) is

$$
\omega^{12}=-\omega^{21}=F_{a} e^{3}+\left(\omega_{2 \mathrm{D}}\right)^{12}, \quad \omega^{23}=-\omega^{32}=-F_{a} e^{1}, \quad \omega^{31}=-\omega^{13}=-F_{a} e^{2}
$$


where we have defined

$$
F_{a}(z, \bar{z}) \equiv \frac{i\left(\partial_{\bar{z}} a-\partial_{z} \bar{a}\right)}{c^{2}}, \quad\left(\omega_{2 \mathrm{D}}\right)^{12}=-\left(\omega_{2 \mathrm{D}}\right)^{21}=-\frac{i}{c}\left(\partial_{z} c d z-\partial_{\bar{z}} c d \bar{z}\right)
$$

with $\omega_{2 \mathrm{D}}$ being the spin connection associated to $e^{1}, e^{2}$ for the $2 \mathrm{D}$ metric $c^{2} d z d \bar{z}$. Note that $F_{a}$ is independent of the choice of chart, while $\omega_{2 \mathrm{D}}$ is not. We have on spinors that

$$
\nabla_{\mu}=\partial_{\mu}-\frac{i}{2} F_{a} \gamma_{\mu} \cdot+i\left(F_{a} e_{\mu}^{3}+\frac{1}{2}\left(\omega_{2 \mathrm{D}}\right)_{\mu}^{12}\right) \gamma^{3}
$$

(cf. (A.33)), where the dots indicate matrix multiplication rather than spinor contraction (see appendix A.3). Hence if we take

$$
H=-i F_{a}, \quad A^{(R)}=-\left(F_{a} e^{3}+\frac{1}{2}\left(\omega_{2 \mathrm{D}}\right)^{12}\right),
$$

then the generalized Killing spinor equations (6.1) are solved by

$$
\xi=x\left(\begin{array}{l}
1 \\
0
\end{array}\right), \quad \tilde{\xi}=x\left(\begin{array}{l}
0 \\
1
\end{array}\right)
$$

in a basis where $\gamma^{a}=x \sigma^{a} x^{-1}$ (here, as in the definition of $K^{\mu}$, we really mean the commuting spinors $\left.\xi\right|_{0}$ and $\left.\tilde{\xi}\right|_{0}$ ). In particular, $\xi, \tilde{\xi}$ are constant in the chosen frame, and since $x \in \mathrm{SU}(2)$, we have both $\tilde{\xi}=\xi^{\dagger}$ and $\left.\left.\xi\right|_{0} \xi^{\dagger}\right|_{0}=1$. Regardless of basis, we have

$$
K^{a}=\left(\left.\xi\right|_{0}\right) \gamma^{a}\left(\left.\tilde{\xi}\right|_{0}\right)=(0-1) \gamma^{a}\left(\begin{array}{l}
0 \\
1
\end{array}\right)=\delta^{a 3},
$$

so that $K=\partial_{\tilde{\psi}}$.

\subsection{Localizing "Seifert" loops}

We now describe how bulk $V_{\mu}=0$ SUSY' restricts to BPS Wilson loops. To summarize, our assumption that $M^{3}$ admits a real, nowhere vanishing Killing vector restricts it to be a Seifert manifold. On any such manifold, it is possible to define a $3 \mathrm{D} \mathcal{N}=2$ supergravity background with $V_{\mu}=0$, in which the Killing spinors take a simple form. Namely, we work in local coordinates $(\tilde{\psi}, z, \bar{z})$ such that $K=\partial_{\tilde{\psi}}$ and the metric takes the standard form (6.8): upon choosing the frame (6.9) and the background fields $H$ and $A^{(R)}$ as in (6.13), the generalized Killing spinor equation $D_{\mu} \eta=-\frac{1}{2} H \gamma_{\mu} \eta$ (with $D_{\mu}$ as in (6.4)) has solutions $\eta=\xi, \tilde{\xi}$ of $R$-charge \pm 1 as in (6.14).

However, the integral curves of the Killing vector field may not be compact. Therefore, local coordinates adapted to the Killing vector do not necessarily define a Seifert fibration of $M^{3}$. Thus the Wilson loops that we consider, while supported on the Seifert manifold $M^{3}$, are not necessarily Seifert loops. The quotation marks in the title of this subsection serve to emphasize that the term "Seifert loop" (in the sense of [14]) is a misnomer.

To begin, consider a Euclidean $3 \mathrm{D} \mathcal{N}=2$ Wilson loop along a curve $\gamma[49,55]$ :

$$
W=\operatorname{Tr}_{R} P \exp \left[i \oint_{\gamma}\left(A_{\mu} d x^{\mu}-i \sigma d s\right)\right]=\operatorname{Tr}_{R} P \exp \left[i \oint_{\gamma} d \tau\left(A_{\mu} \dot{x}^{\mu}-i \sigma|\dot{x}|\right)\right] \text {. }
$$


The BPS conditions following from (6.2) take the same form on any background geometry:

$$
n^{\mu} \gamma_{\mu} \xi-\xi=0, \quad n^{\mu} \gamma_{\mu} \tilde{\xi}+\tilde{\xi}=0
$$

with $n^{\mu}=\dot{x}^{\mu} /|\dot{x}|$ being the unit tangent vector to $\gamma$. They are satisfied when $n^{\mu}=-K^{\mu}$. Hence a BPS Wilson loop preserving both supercharges under consideration lies along an integral curve of $K^{\mu}{ }^{20}$

To determine how bulk SUSY' restricts to these BPS Wilson loops, note that even after demanding that the Killing spinors $\xi, \tilde{\xi}$ be properly normalized, we still have the freedom to introduce a relative phase between them (the overall phase is immaterial). Therefore, let us keep $\xi$ as in (6.14), with $K^{\mu}=\xi \gamma^{\mu} \xi^{\dagger}$, and write

$$
\tilde{\xi}=\rho x\left(\begin{array}{l}
0 \\
1
\end{array}\right)=\rho \xi^{\dagger}, \quad|\rho|=1 .
$$

The linear combinations of 3D fermions that appear in the 1D multiplets depend on the gamma matrix conventions. For simplicity, we work in the basis $\gamma^{a}=\sigma^{a}(a=1,2,3)$. According to the above discussion, we fix $\left(n^{1}, n^{2}, n^{3}\right)=(0,0,-1)$. Restoring Grassmann parameters, we have

$$
\left(\begin{array}{l}
\xi_{1} \\
\xi_{2}
\end{array}\right)=\left(\begin{array}{c}
\omega \\
0
\end{array}\right), \quad\left(\begin{array}{c}
\tilde{\xi}_{1} \\
\tilde{\xi}_{2}
\end{array}\right)=\left(\begin{array}{c}
0 \\
\rho \bar{\omega}
\end{array}\right) .
$$

To restrict the SUSY' transformations (6.2) and (6.3), we drop dependence on the 1 and 2 directions and consider only the component of the gauge field along the loop. Along the loop, frame and spacetime indices are equivalent since $e_{3}^{3}=1$. For the vector multiplet, it is convenient to define the 1D SUSY'-covariant derivative

$$
D_{3}^{\prime}(\cdot) \equiv \partial_{3}(\cdot)-i\left[A_{3}+i \sigma,(\cdot)\right]
$$

on both scalars and spinors, which satisfies $\delta^{\prime} D_{3}^{\prime}(\cdot)=D_{3}^{\prime} \delta^{\prime}(\cdot)$ and $D_{3}^{\prime} \sigma=D_{3} \sigma$. Note that $D_{3}^{\prime}(\cdot)$ and $D_{3}(\cdot)+[\sigma,(\cdot)]$ coincide on scalars, but not on spinors; note also that in $1 \mathrm{D}$, we need not diffeomorphism-covariantize the derivative acting on spinors because the spin connection is trivial. In our supergravity background and frame, we have on spinors that

$$
\nabla_{i}=\partial_{i}-\frac{1}{2} H \gamma_{i}+i A_{i}^{(R)} \gamma_{3} \Longrightarrow \nabla_{3} \psi_{i_{\perp}}=\partial_{3} \psi_{i_{\perp}}-(-1)^{i_{\perp}}\left(\frac{1}{2} H-i A_{3}^{(R)}\right) \psi_{i_{\perp}}
$$

where $i_{\perp}=1,2$. Moreover, it follows from the $V_{\mu}=0$ SUSY' algebra that the gauginos $\lambda, \tilde{\lambda}$ have $R$-charges $\mp 1$, so (6.4) and (6.21) give

$$
D_{3} \lambda_{1}=\partial_{3} \lambda_{1}+\frac{1}{2} H \lambda_{1}-i\left[A_{3}, \lambda_{1}\right], \quad D_{3} \tilde{\lambda}_{2}=\partial_{3} \tilde{\lambda}_{2}-\frac{1}{2} H \tilde{\lambda}_{2}-i\left[A_{3}, \tilde{\lambda}_{2}\right] .
$$

\footnotetext{
${ }^{20}$ These conditions would be equivalent in Lorentzian signature (see section 5).
} 
Specializing to our specific $\xi, \tilde{\xi}$, we obtain from (6.2) (using (6.20) and (6.22)) that

$$
\begin{aligned}
\delta^{\prime} \sigma & =-\left(\omega \tilde{\lambda}_{2}+\rho \bar{\omega} \lambda_{1}\right), \\
\delta^{\prime} A_{3} & =i\left(\omega \tilde{\lambda}_{2}+\rho \bar{\omega} \lambda_{1}\right), \\
\delta^{\prime} \lambda_{1} & =-i \omega D+i \omega D_{3}^{\prime} \sigma+i \omega \sigma H, \\
\delta^{\prime} \tilde{\lambda}_{2} & =i \rho \bar{\omega} D+i \rho \bar{\omega} D_{3}^{\prime} \sigma-i \rho \bar{\omega} \sigma H, \\
\delta^{\prime} D & =-\left(\omega D_{3}^{\prime} \tilde{\lambda}_{2}-\rho \bar{\omega} D_{3}^{\prime} \lambda_{1}\right),
\end{aligned}
$$

with $\delta^{\prime} \lambda_{2}=\delta^{\prime} \tilde{\lambda}_{1}=0$. We thus obtain the following restricted multiplets in 1D:

- A $1 \mathrm{D}$ vector $\left\{0,0, A_{3}+i \sigma\right\}$ where $\delta^{\prime}\left(A_{3}+i \sigma\right)=0$.

- Two independent 1D adjoint chirals (not related by complex conjugation) $\left\{0, \lambda_{2}\right\}$ and $\left\{0, \tilde{\lambda}_{1}\right\}$ where $\delta^{\prime} \lambda_{2}=\delta^{\prime} \tilde{\lambda}_{1}=0$.

The remaining fields do not comprise good multiplets for any choice of $\rho$. Now note that the Euclidean SUSY' transformation rules of a $1 \mathrm{D}$ chiral are $\delta \phi=\epsilon \psi, \delta \psi=2 \epsilon^{\dagger} \dot{\phi}$. It turns out that for a $3 \mathrm{D}$ chiral to restrict to a $1 \mathrm{D}$ chiral, we must choose $\rho=i$. Indeed, consider a fundamental chiral multiplet of dimension $\Delta$. The corresponding 1D SUSY'-covariant derivative is

$$
D_{3}^{\prime}(\cdot) \equiv \partial_{3}(\cdot)-i\left(A_{3}+i \sigma\right)(\cdot)
$$

which satisfies $\delta^{\prime} D_{3}^{\prime}(\cdot)=D_{3}^{\prime} \delta^{\prime}(\cdot)$. From the $V_{\mu}=0$ SUSY' algebra, we see that $A, \psi, F$ have $R$-charges $-\Delta, 1-\Delta, 2-\Delta$, respectively, so that

$$
\begin{aligned}
D_{3} A & =\left(\partial_{3}-i A_{3}+i \Delta A_{3}^{(R)}\right) A, \\
D_{3} \psi_{1} & =\nabla_{3} \psi_{1}-i A_{3} \psi_{1}-i(1-\Delta) A_{3}^{(R)} \psi_{1}
\end{aligned}
$$

with $\nabla_{3} \psi_{1}$ as in (6.21). Substituting our specific $\xi, \tilde{\xi}$ into (6.3) and using (6.24) then gives the restricted transformation rules

$$
\begin{aligned}
\delta^{\prime} A & =-\sqrt{2} \omega \psi_{2}, \\
\delta^{\prime} \psi_{1} & =-\sqrt{2} \omega F \\
\delta^{\prime} \psi_{2} & =i \sqrt{2} \rho \bar{\omega}\left(D_{3}^{\prime}+i \Delta A_{3}^{(R)}-\Delta H\right) A \\
\delta^{\prime} F & =i \sqrt{2} \rho \bar{\omega}\left(D_{3}^{\prime}-i(2-\Delta) A_{3}^{(R)}+H\right) \psi_{1}+2 i \rho \bar{\omega} \tilde{\lambda}_{1} A-i \sqrt{2} \Delta \rho \bar{\omega} \psi_{1} H .
\end{aligned}
$$

Choosing both $\rho=i$ and $\Delta=0$, we obtain a single restricted multiplet in $1 \mathrm{D}$, namely a 1D fundamental chiral $\left\{A,-\sqrt{2} \psi_{2}\right\}$ where

$$
\begin{aligned}
\delta^{\prime} A & =\omega\left(-\sqrt{2} \psi_{2}\right), \\
\delta^{\prime}\left(-\sqrt{2} \psi_{2}\right) & =2 \bar{\omega} D_{3}^{\prime} A .
\end{aligned}
$$

The remaining transformation rules superficially resemble those of a 1D fundamental antichiral, but they do not close and do not have the correct relative sign. 
The key point is that the transformation rules for the restricted 1D multiplets are independent of the supergravity background fields and take exactly the same form as in flat space. The fields are a priori complex, and $D_{3}^{\prime}$ is not Hermitian because it involves a complexified gauge field. After imposing reality conditions in the path integral, we want $\sigma$ purely imaginary, $A_{3}$ purely real, and $D$ purely imaginary; the fermions remain independent.

\subsubsection{Example: $S^{3}$}

Let us see how this setup works in the familiar setting of $S^{3}$, whose radius we take to be $\ell$. We coordinatize $S^{3}$ by $g \in \mathrm{SU}(2)$, which admits both left and right actions of $\mathrm{SU}(2)$. Frame indices are identified with $\mathfrak{s u}(2)$ indices in the basis $T^{a}=\sigma^{a} / 2$. The $\mathfrak{s u}(2)$-valued left- and right-invariant one-forms are

$$
\Omega_{L} \equiv g^{-1} d g=i\left(\Omega_{L}\right)^{a} T^{a}, \quad \Omega_{R} \equiv d g g^{-1}=i\left(\Omega_{R}\right)^{a} T^{a} .
$$

The bi-invariant Riemannian metric is

$$
d s^{2}=\frac{\ell^{2}}{2} \operatorname{Tr}\left(d g \otimes d g^{-1}\right)=-\frac{\ell^{2}}{2} \operatorname{Tr}\left(\Omega_{L}^{\otimes 2}\right)=-\frac{\ell^{2}}{2} \operatorname{Tr}\left(\Omega_{R}^{\otimes 2}\right),
$$

and the frame one-forms satisfying $e_{\mu}^{a} e_{\nu}^{b} \delta_{a b}=g_{\mu \nu}$ are

$$
e_{L}=\frac{\ell}{2} \Omega_{L}, \quad e_{R}=\frac{\ell}{2} \Omega_{R} .
$$

The spin connection $\omega^{a b} \equiv \omega_{\mu}^{a b} d x^{\mu}$ in the two frames is $\left(\omega_{L}\right)^{a b}=\frac{1}{\ell} \epsilon^{a b c}\left(e_{L}\right)^{c}$ and $\left(\omega_{R}\right)^{a b}=$ $-\frac{1}{\ell} \epsilon^{a b c}\left(e_{R}\right)^{c}$. Hence on spinors (see (A.33)), we have

$$
\left.\nabla_{\mu}\right|_{\mathrm{LI}}=\partial_{\mu}+\frac{i}{2 \ell} \gamma_{\mu} \cdot,\left.\quad \nabla_{\mu}\right|_{\mathrm{RI}}=\partial_{\mu}-\frac{i}{2 \ell} \gamma_{\mu} .
$$

in the left- and right-invariant frames, respectively. The four $\mathbb{C}$-linearly independent conformal Killing spinor fields on $S^{3}$ can be constructed by taking $\xi$ constant in the left-invariant frame or constant in the right-invariant frame:

$$
\nabla_{\mu} \xi= \pm \frac{i}{2 \ell} \gamma_{\mu} \cdot \xi=\mp \frac{i}{2 \ell} \gamma_{\mu} \xi \Longleftrightarrow \xi^{\prime}=\mp \frac{i}{2 \ell} \xi,
$$

with two solutions for each sign. Keeping in mind that (A.32) means $\nabla_{\mu} \xi=-\gamma_{\mu} \cdot \xi^{\prime}$, we refer to spinors with $\xi^{\prime}=-\frac{i}{2 \ell} \xi$ as "positive" and those with $\xi^{\prime}=\frac{i}{2 \ell} \xi$ as "negative." These conformal Killing spinors are genuine Killing spinors, for which $\xi^{\prime} \propto \xi$. For these spinors, the condition (A.40) for closure of the superconformal algebra is automatically satisfied.

By taking the spinor parameters $\xi, \tilde{\xi}$ of the superconformal algebra both to be positive or negative (hence constant in the left- or right-invariant frame), the dilatation parameter $\rho=\frac{2 i}{3} \nabla_{\mu}\left(\xi \gamma^{\mu} \tilde{\xi}\right)$ vanishes and we restrict to either of the non-conformal subalgebras

$$
\mathfrak{o s p}(2 \mid 2)_{\text {left }} \times \mathfrak{s u}(2)_{\text {right }}, \mathfrak{s u}(2)_{\text {left }} \times \mathfrak{o s p}(2 \mid 2)_{\text {right }} \subset \mathfrak{o s p}(2 \mid 2,2),
$$

which are $S^{3}$ analogues of $\mathcal{N}=2$ Poincaré supersymmetry in $\mathbb{R}^{3}$. The Killing vector $\xi \gamma^{\mu} \tilde{\xi}$ is likewise constant in the appropriate frame. The supercharges generate $\mathfrak{o s p}(2 \mid 2)$ (left or 
right), whose bosonic subalgebra contains the $\mathfrak{s u}(2)$ (left or right) isometry and the $\mathfrak{u}(1)_{R}$. The SUSY' transformations become

$$
\begin{aligned}
\delta^{\prime} \sigma & =-(\xi \tilde{\lambda}-\tilde{\xi} \lambda), \\
\delta^{\prime} A_{\mu} & =i\left(\xi \gamma_{\mu} \tilde{\lambda}+\tilde{\xi} \gamma_{\mu} \lambda\right), \\
\delta^{\prime} \lambda & =-i \xi D-i \gamma^{\mu} \xi D_{\mu} \sigma-\frac{i}{2} \sqrt{g}^{-1} \epsilon^{\mu \nu \rho} \gamma_{\rho} \xi F_{\mu \nu} \mp \frac{1}{\ell} \sigma \xi \\
\delta^{\prime} \tilde{\lambda} & =i \tilde{\xi} D+i \gamma^{\mu} \tilde{\xi} D_{\mu} \sigma-\frac{i}{2} \sqrt{g}^{-1} \epsilon^{\mu \nu \rho} \gamma_{\rho} \tilde{\xi} F_{\mu \nu} \pm \frac{1}{\ell} \sigma \tilde{\xi}, \\
\delta^{\prime} D & =-\left(\xi \gamma^{\mu} D_{\mu} \tilde{\lambda}-\tilde{\xi} \gamma^{\mu} D_{\mu} \lambda\right)+[\xi \tilde{\lambda}+\tilde{\xi} \lambda, \sigma] \mp \frac{i}{2 \ell}(\xi \tilde{\lambda}-\tilde{\xi} \lambda)
\end{aligned}
$$

for the vector multiplet and

$$
\begin{aligned}
\delta^{\prime} A & =-\sqrt{2} \xi \psi \\
\delta^{\prime} \psi & =-\sqrt{2} \xi F+i \sqrt{2} \gamma^{\mu} \tilde{\xi} D_{\mu} A+i \sqrt{2} \tilde{\xi} \sigma A \pm \frac{\sqrt{2} \Delta}{\ell} \tilde{\xi} A \\
\delta^{\prime} F & =i \sqrt{2} \tilde{\xi} \gamma^{\mu} D_{\mu} \psi-i \sqrt{2} \sigma \tilde{\xi} \psi-2 i \tilde{\xi} \tilde{\lambda} A \mp \frac{\sqrt{2}(\Delta-1 / 2)}{\ell} \tilde{\xi} \psi
\end{aligned}
$$

for a fundamental chiral multiplet of dimension $\Delta$, with the top and bottom signs corresponding to $\xi, \tilde{\xi}$ positive and $\xi, \tilde{\xi}$ negative, respectively. The non-conformal $\mathcal{N}=2$ Yang-Mills Lagrangian is invariant under this restricted supersymmetry. Indeed, we find that (6.7) holds, where $\left.\mathcal{L}_{\mathrm{YM}}\right|_{S^{3}}$ corresponds to taking $H= \pm i / \ell$ with the top and bottom signs as above:

$$
\left.\mathcal{L}_{\mathrm{YM}}\right|_{S^{3}}=\operatorname{Tr}\left[\frac{1}{4} F_{\mu \nu} F^{\mu \nu}+\frac{1}{2} D_{\mu} \sigma D^{\mu} \sigma-\frac{1}{2}\left(D \mp \frac{i \sigma}{\ell}\right)^{2}-i \tilde{\lambda} \gamma^{\mu} D_{\mu} \lambda+i \tilde{\lambda}[\sigma, \lambda] \mp \frac{1}{2 \ell} \lambda \tilde{\lambda}\right] .
$$

A fortiori, $\left.\mathcal{L}_{\mathrm{YM}}\right|_{S^{3}}$ is supersymmetric with respect to the restricted SUSY'.

One typically restricts to the subalgebra of positive ("left-invariant") spinors and uses as a localizing term $\left.\mathcal{L}_{\mathrm{YM}}\right|_{S^{3}}$ with $H=i / \ell$. Let us instead see how the general construction of section 6.2 reduces to the known one in the case of $S^{3}$. In a form that makes the Hopf fibration manifest [39], the metric (6.29) on $S^{3}$ is

$$
d s^{2}=\frac{\ell^{2}}{4}\left(d \theta^{2}+\sin ^{2} \theta d \phi^{2}+(d \psi+\cos \theta d \phi)^{2}\right), \theta \in[0, \pi), \phi \in[0,2 \pi), \psi \in[0,4 \pi),
$$

where the first two terms are the round metric on $S^{2}$ of radius $\ell / 2 .{ }^{21}$ Defining the dimensionful variable $\tilde{\psi}=\ell \psi / 2$, so that $\tilde{\psi} / \ell \in[0,2 \pi)$, we have $K=\partial_{\tilde{\psi}}$. Stereographic projection gives the relation $z=\ell e^{i \phi} / \tan (\theta / 2)$ between complex coordinates $z, \bar{z}$ and spherical coordinates $(\theta, \phi)$ on $S^{2}$. To go from the patch containing the origin to the patch containing $\infty$ on $S^{2}$, we simply take $z^{\prime}=\ell^{2} / z$ ( $\tilde{\psi}$ does not transform). These correspond to adapted coordinates (6.8) on $S^{3}$ with

$$
a=\frac{i \ell}{4 z}\left(\frac{1-|z|^{2} / \ell^{2}}{1+|z|^{2} / \ell^{2}}\right), c=\frac{1}{1+|z|^{2} / \ell^{2}} \Longrightarrow H=-\frac{i}{\ell}, A^{(R)}=-\frac{d \tilde{\psi}}{\ell}-\frac{i}{4}\left(\frac{d z}{z}-\frac{d \bar{z}}{\bar{z}}\right) .
$$

\footnotetext{
${ }^{21}$ The integral curves of Killing vectors on $S^{3}$ are great circles corresponding to fibers of the Hopf fibration $S^{3} \rightarrow S^{2}$. The fiber over a given point on $S^{2}$ is the locus with fixed $(\theta, \phi)$ and arbitrary $\psi$.
} 
Despite that the resulting SUSY' algebra takes the same form as in the right-invariant frame ((6.33) and (6.34) with the bottom sign), our standard frame (6.9) for the adapted coordinates (6.8) is neither the left- nor the right-invariant frame on $S^{3}$. Indeed, in the $L$ and $R$ frames, we may choose $A^{(R)}=0$. To reproduce the analysis in the left- or rightinvariant frame directly from adapted coordinates, one can work in toroidal rather than Hopf coordinates, as we do on $S_{b}^{3}$.

Finally, we recall that expectation values of half-BPS Wilson loops in the $\mathcal{N}=2 G_{k}$ theory on $S^{3}$ can be written as [15]

$$
\left\langle W_{R_{1}} \cdots W_{R_{n}}\right\rangle=\frac{1}{|\mathcal{W}| Z} \int_{\mathfrak{t}} d \hat{\sigma}_{0} e^{-k \pi i \operatorname{Tr} \hat{\sigma}_{0}^{2}} \prod_{i=1}^{n} \operatorname{Tr}_{R_{i}}\left(e^{2 \pi \hat{\sigma}_{0}}\right) \prod_{\alpha} 2 \sinh \left(\pi \alpha\left(\hat{\sigma}_{0}\right)\right),
$$

where $\hat{\sigma}_{0}$ lies in a Cartan subalgebra $\mathfrak{t} \subset \mathfrak{g}$ and $\alpha$ ranges over the roots of $\mathfrak{g}$. This is a sum of Gaussian integrals that can be evaluated to reproduce the $\mathcal{N}=0$ result, up to a framing phase and a level shift.

\subsubsection{Example: $S_{b}^{3}$}

Consider parametrizing the round sphere by toroidal coordinates, which manifest $S^{3}$ as a torus fibered over a closed interval $[39,46]$. We regard $S^{3}$ as the locus $(u, v) \in \mathbb{C}^{2}$ satisfying $|u|^{2}+|v|^{2}=1$, but rather than parametrizing $u, v$ using Euler angles, we use coordinates $\chi \in[0, \pi / 2]$ and $\phi_{1}, \phi_{2} \in[0,2 \pi)$ :

$$
u=\cos \chi e^{i \phi_{1}}, \quad v=\sin \chi e^{i \phi_{2}}, \quad d s^{2}=\ell^{2}\left(d \chi^{2}+\cos ^{2} \chi d \phi_{1}^{2}+\sin ^{2} \chi d \phi_{2}^{2}\right) .
$$

This metric clearly admits two independent $U(1)$ isometries. More generally, consider the following squashed-sphere metric, which preserves a $\mathrm{U}(1) \times \mathrm{U}(1)$ subgroup of the $\mathrm{SU}(2) \times$ $\mathrm{SU}(2)$ isometry group of $S^{3}$ :

$$
d s^{2}=f(\chi)^{2} d \chi^{2}+\ell_{1}^{2} \cos ^{2} \chi d \phi_{1}^{2}+\ell_{2}^{2} \sin ^{2} \chi d \phi_{2}^{2} .
$$

Here, $\ell_{1}, \ell_{2}>0$ and $f$ is a smooth, positive function on $[0, \pi / 2]$ satisfying $f(0)=\ell_{2}$ and $f(\pi / 2)=\ell_{1}$ to avoid conical singularities along the $\phi_{1}$ and $\phi_{2}$ circles, respectively. The squashing parameter of this geometry is $b \equiv \sqrt{\ell_{1} / \ell_{2}}$. A generic Killing vector is a linear combination of the $\mathrm{U}(1)$ generators: $K=\alpha \partial_{\phi_{1}}+\beta \partial_{\phi_{2}}$. To use the $3 \mathrm{D} \mathcal{N}=2$ supergravity background described above, we take

$$
|K|^{2}=\alpha^{2} \ell_{1}^{2} \cos ^{2} \chi+\beta^{2} \ell_{2}^{2} \sin ^{2} \chi=1 \Longleftrightarrow K=\ell_{1}^{-1} \partial_{\phi_{1}}+\ell_{2}^{-1} \partial_{\phi_{2}}
$$

and define local coordinates $(z=x+i y)$

$$
x=\int^{\chi} \frac{f\left(\chi^{\prime}\right)}{\sin \chi^{\prime} \cos \chi^{\prime}} d \chi^{\prime}, \quad y=\ell_{1} \phi_{1}-\ell_{2} \phi_{2}, \quad \tilde{\psi}=\ell_{1} \phi_{1} \cos ^{2} \chi+\ell_{2} \phi_{2} \sin ^{2} \chi,
$$

in terms of which the metric (6.39) on $S_{b}^{3}$ can be written in the standard form (6.8) with

$$
a=2 \sin \chi \cos \chi\left(\ell_{1} \phi_{1}-\ell_{2} \phi_{2}\right) d \chi, \quad c=\sin \chi \cos \chi .
$$


In addition, $K=\partial_{\tilde{\psi}}$. In these coordinates, (6.9) yields the vielbein

$$
e^{1}=f(\chi) d \chi, \quad e^{2}=-\sin \chi \cos \chi\left(\ell_{1} d \phi_{1}-\ell_{2} d \phi_{2}\right), e^{3}=\ell_{1} \cos ^{2} \chi d \phi_{1}+\ell_{2} \sin ^{2} \chi d \phi_{2} .
$$

The corresponding background field configuration is

$$
H=\frac{i}{f}, \quad V_{\mu}=0, \quad A^{(R)}=\frac{\ell_{1} d \phi_{1}+\ell_{2} d \phi_{2}}{2 f} .
$$

We may fix a gauge in which $A^{(R)}$ is regular everywhere:

$$
A^{(R)}=\frac{1}{2}\left(\frac{\ell_{1}}{f}-1\right) d \phi_{1}+\frac{1}{2}\left(\frac{\ell_{2}}{f}-1\right) d \phi_{2} .
$$

Performing the corresponding $R$-symmetry gauge transformation on the Killing spinors in (6.14), we have in the chosen frame and gauge that

$$
\xi=e^{i\left(\phi_{1}+\phi_{2}\right) / 2}\left(\begin{array}{l}
1 \\
0
\end{array}\right), \quad \tilde{\xi}=e^{-i\left(\phi_{1}+\phi_{2}\right) / 2}\left(\begin{array}{l}
0 \\
1
\end{array}\right) .
$$

For generic $b$, the metric in $\tilde{\psi}, z, \bar{z}$ coordinates does not define an $S^{1}$ fibration because the coordinate $\tilde{\psi}$ is not periodic: namely, we see from (6.40) that the integral curves of $K$ do not close on the tori at $\chi \neq 0, \pi / 2$ unless $b^{2}=\ell_{1} / \ell_{2}$ is rational. If $b^{2}=m / n$ with $m, n$ relatively prime integers, then the integral curves for $\chi \neq 0, \pi / 2$ are $(n, m)$ torus knots wrapping the $\phi_{1}$ cycle $n$ times and the $\phi_{2}$ cycle $m$ times. These curves have length

$$
2 \pi\left(\ell_{1}^{2} n^{2} \cos ^{2} \chi+\ell_{2}^{2} m^{2} \sin ^{2} \chi\right)^{1 / 2}=2 \pi \sqrt{\ell_{1} \ell_{2} m n},
$$

regardless of $\chi$. On the other hand, at $\chi=0, \pi / 2$, the vector field $K$ is singular and its integral curves are circles (regardless of $b$ ) of lengths $2 \pi \ell_{1}$ and $2 \pi \ell_{2}$, respectively. One can insert supersymmetric Wilson loops along these knots or circles.

The round-sphere limit is given by $f(\chi)=\ell_{1}=\ell_{2}=\ell$. In this limit, we have $H=i / \ell$ and $V_{\mu}=A_{\mu}^{(R)}=0$, and both the generalized Killing spinor equations and the $V_{\mu}=0$ SUSY' transformations reduce to those for the left-invariant frame on $S^{3}$. Note that the $\mathfrak{s u}(1 \mid 1)$ algebra contains half of the Killing spinors that generate $\mathfrak{o s p}(2 \mid 2)_{\text {left }}$ on $S^{3}$; the existence of two additional left-invariant Killing spinors is due to the extra symmetry of $S^{3}$. Recall that on $S^{3}$, the available spinors in $\mathfrak{o s p}(2 \mid 2)_{\text {left }}$ are halved by the BPS condition for a half-BPS Wilson loop; it then suffices to use one of the two remaining supercharges for localization.

Cohomological $[40,52]$ or index theory $[39,66]$ arguments can be used to derive the partition function of pure $\mathcal{N}=2$ Chern-Simons theory on $S_{b}^{3}$ :

$$
Z_{S_{b}^{3}}=\frac{1}{|\mathcal{W}|} \int_{\mathfrak{t}} d \hat{\sigma}_{0} e^{-k \pi i \operatorname{Tr} \hat{\sigma}_{0}^{2}} \prod_{\alpha>0} 4 \sinh \left(\pi b \alpha\left(\hat{\sigma}_{0}\right)\right) \sinh \left(\pi b^{-1} \alpha\left(\hat{\sigma}_{0}\right)\right) .
$$

Again by (6.17), BPS Wilson loops lie along integral curves of $K^{\mu}$ and thus correspond to insertions of

$$
W\left[\hat{\sigma}_{0}\right]=\operatorname{Tr}_{R} \exp \left(\frac{\hat{\sigma}_{0}}{\ell} \oint_{\gamma} d s\right)=\sum_{\rho \in R} \begin{cases}e^{2 \pi b \rho\left(\hat{\sigma}_{0}\right)} & \text { if } \chi=0, \\ e^{2 \pi b^{-1} \rho\left(\hat{\sigma}_{0}\right)} & \text { if } \chi=\pi / 2, \\ e^{2 \pi \sqrt{m n} \rho\left(\hat{\sigma}_{0}\right)} & \text { if } b^{2}=m / n, \operatorname{gcd}(m, n)=1, \chi \neq 0, \pi / 2\end{cases}
$$


in the partition function (here, $\ell \equiv \sqrt{\ell_{1} \ell_{2}}$ ). Clearly, this matrix model reduces to that for $S^{3}$ when $b=1$. One can use this matrix model to calculate the Jones polynomial for torus knots and torus links [54] (see also [14, 33]). To do so, one must account for supersymmetric framing: the self-linking number at generic $\chi$ is given by the linking number of nested torus knots, while circular Wilson loops at $\chi=0, \pi / 2$ have fractional self-linking number.

\subsubsection{Non-example: (squashed) lens spaces}

One might wish to apply the formalism of section 6.2 to more general Seifert manifolds (for useful references, see $[2,13,14]$ ). Lens spaces provide a nice class of examples: these are Seifert manifolds that admit infinitely many distinct Seifert fibrations.

For relatively prime integers $p, q$, the lens space $L(p, q)$ can be defined as the quotient of $S^{3}$, namely the locus $(u, v) \in \mathbb{C}^{2}$ where $|u|^{2}+|v|^{2}=1$, by the free $\mathbb{Z}_{p}$ action

$$
(u, v) \mapsto\left(e^{2 \pi i / p} u, e^{-2 \pi i q / p} v\right) .
$$

More generally, one obtains a space $L_{b}(p, q)$ that is topologically $L(p, q)$ by starting with a metric (6.39) for $S_{b}^{3}$ and imposing the identifications

$$
\left(\chi, \phi_{1}, \phi_{2}\right) \sim\left(\chi, \phi_{1}+2 \pi / p, \phi_{2}-2 \pi q / p\right) .
$$

Aside from the case of $L(p,-1)$ [57], lens spaces cannot be written as Seifert manifolds in such a way that the base is a smooth Riemann surface, without orbifold points (the analysis of [26] is also restricted to Seifert manifolds with a smooth base).

The only case in which it is possible to localize on a squashed lens space $L_{b}(p, q)$ using the $V_{\mu}=0$ supergravity background (6.44) on $S_{b}^{3}$ is that of $L_{b}(p, 1)$, because only in this case does the $\mathbb{Z}_{p}$ action (6.48) preserve the Killing spinors (6.46).

On $L_{b}(p, 1)$, the values of the supergravity background fields and the BPS equations are the same as on $S_{b}^{3}$. However, the localization locus is different because $\pi_{1}\left(L_{b}(p, 1)\right)=\mathbb{Z}_{p}$. Upon summing over holonomies $\mathfrak{m} \in \Lambda_{W}^{\vee} / p \Lambda_{W}^{\vee}$ where $\Lambda_{W}^{\vee} \subset \mathfrak{t}$ is the coweight lattice of $G[56,67]$, the partition function of $\mathcal{N}=2$ Chern-Simons theory on $L_{b}(p, 1)$ is

$$
Z_{L_{b}(p, 1)}=\frac{1}{|\mathcal{W}|} \sum_{\mathfrak{m}} \int_{\mathfrak{t}} d \hat{\sigma}_{0} e^{-\frac{k \pi i}{p} \operatorname{Tr}\left(\hat{\sigma}_{0}^{2}-\mathfrak{m}^{2}\right)} \prod_{\alpha>0} \prod_{ \pm} 2 \sinh \left(\pi b^{ \pm 1} \alpha\left(\hat{\sigma}_{0} \pm i \mathfrak{m}\right) / p\right)
$$

One can obtain from this matrix model the expectation value of a Wilson loop wrapping the non-contractible cycle (see [56] and references therein).

Now suppose we want to localize Wilson loops on $L_{b}(p, 1)$ with $b^{2}$ rational, so that the orbits of the Killing vector close into torus knots. For any Wilson loop along a torus knot in $S_{b}^{3}$, one can easily compute the expectation value of its image in $L_{b}(p, 1)$ by writing down the appropriate matrix model. One could in principle take a different approach to localizing Wilson loops on $L_{b}(p, q)$, which is more akin to that in [57]: instead of viewing $L_{b}(p, q)$ as a quotient of $S_{b}^{3}$, exhibit it directly as a Seifert fibration and consider loops wrapping the circle fibers (for rational $b^{2}$, the $L_{b}(p, q)$ are Seifert fibrations over $S^{2}$ with two singular fibers). This was accomplished in [57] for the case of round $L(p,-1)$. 
In the special case of $S^{2} \times S^{1}=L(0,1)$, the $V_{\mu}=0$ background (with $K$ generating translations along the $S^{1}$ ) computes the topologically twisted index [34] with a negative unit of $R$-symmetry flux through the $S^{2}$. One can place Wilson loops along the $S^{1}$ over any point on the $S^{2}$.

\subsection{Localization on solid torus}

In all compact manifolds that we have considered, the half-BPS sector includes a very limited subset of the possible loop observables. It is natural to ask whether the localization argument can be applied to a basic "building block" that can then be used to assemble Wilson loop configurations of more complicated topologies. The natural candidate for such a building block is a solid torus [33, 68]. Here, we take only the most tentative possible step toward making this program precise by localizing on a solid torus dressed by a Wilson loop. This constructive approach has appeared in the supersymmetric context in the guise of holomorphic blocks [60,61], and also as a gluing formula of a suggestively similar form to surgery in pure Chern-Simons theory [57]; however, both of these incarnations depend on more than topological data.

Localization on a solid torus is a simple application of the results of [58]. We restrict our attention to Dirichlet boundary conditions. These boundary conditions eliminate half of the fermions and, with the appropriate choice of localizing term, do not require introducing boundary degrees of freedom. For pure $\mathcal{N}=2$ gauge theory, we can preserve two supersymmetries on the solid torus, which gives us precisely the $1 \mathrm{D} \mathcal{N}=2$ supersymmetry required for cancellation of the line shift.

The solid torus is constructed by starting with toroidal coordinates (6.38) on round $S^{3}$ and restricting to $\chi \in\left[0, \chi_{0}\right]$ with $\chi_{0}<\pi / 2$. In the standard supergravity background (6.44) and frame (6.43) (with $f=\ell_{1}=\ell_{2}=\ell$ ), we may choose a gauge in which $A^{(R)}=0$ and the Killing spinors are as in (6.46). Seeing as $\gamma^{\chi}=\frac{1}{\ell} \gamma^{1}$ in our frame, we have

$$
-\ell e^{-i\left(\phi_{1}+\phi_{2}\right)} \gamma^{\chi} \xi=\tilde{\xi}
$$

(recall that $-\gamma^{\chi} \xi=\gamma^{\chi} \cdot \xi$ ). Up to a slight difference in conventions, this is precisely the condition satisfied by Killing spinors preserved by the boundary conditions of [58] on the solid torus. Specifically, the boundary conditions that we wish to impose are

$$
A_{\tilde{\mu}}\left|=a_{\tilde{\mu}}, \quad \sigma\right|=\sigma_{0}, \quad \ell e^{-i\left(\phi_{1}+\phi_{2}\right)} \gamma^{\chi} \lambda|=\tilde{\lambda}|
$$

where $\tilde{\mu} \in\left\{\phi_{1}, \phi_{2}\right\}$ denotes the directions tangent to the boundary $T^{2}$, "" denotes restriction to the boundary $\chi=\chi_{0}$, and $a_{\tilde{\mu}}$ and $\sigma_{0}$ are constants. The fields $A_{\chi}$ and $D$ are left free. Given (6.50), one easily sees that these boundary conditions are compatible with the relevant SUSY' transformations, which are given by (6.33) with the top sign (note, however, that we are not working in the left-invariant frame).

The boundary terms in the SUSY' variation of the curved-space Chern-Simons action (A.45) are given by (A.46). For the Yang-Mills action on the solid torus, we write the fermion kinetic terms symmetrically between $\lambda$ and $\tilde{\lambda}$ (following [58]) to ensure that 
the boundary conditions kill surface terms without the need to introduce a compensating boundary action. Namely, we use as a localizing term

$$
\left.\left.\mathcal{L}_{(\mathrm{YM})}\right|_{S^{3}} \equiv \mathcal{L}_{\mathrm{YM}}\right|_{S^{3}, \tilde{\lambda} \gamma^{\mu} D_{\mu} \lambda \rightarrow \frac{1}{2}\left(\tilde{\lambda} \gamma^{\mu} D_{\mu} \lambda+\lambda \gamma^{\mu} D_{\mu} \tilde{\lambda}\right)},
$$

where $\left.\mathcal{L}_{\mathrm{YM}}\right|_{S^{3}}$ is defined by choosing the top sign in (6.35) (the parentheses in (YM) are a mnemonic for symmetrization). One computes that the corresponding boundary terms are

$$
\begin{aligned}
\left.\delta^{\prime} \mathcal{L}_{(\mathrm{YM})}\right|_{S^{3}}=\frac{1}{2} \nabla_{\mu} & \operatorname{Tr}\left[\frac{1}{2} \sqrt{g}^{-1} \epsilon^{\mu \nu \rho} F_{\nu \rho}(\xi \tilde{\lambda}+\tilde{\xi} \lambda)+i \sqrt{g}^{-1} \epsilon^{\mu \nu \rho} D_{\nu} \sigma\left(\xi \gamma_{\rho} \tilde{\lambda}-\tilde{\xi} \gamma_{\rho} \lambda\right)\right. \\
& \left.+i F^{\mu \nu}\left(\xi \gamma_{\nu} \tilde{\lambda}+\tilde{\xi} \gamma_{\nu} \lambda\right)-D^{\mu} \sigma(\xi \tilde{\lambda}-\tilde{\xi} \lambda)+\left(D-\frac{i \sigma}{\ell}\right)\left(\xi \gamma^{\mu} \tilde{\lambda}-\tilde{\xi} \gamma^{\mu} \lambda\right)\right] .
\end{aligned}
$$

By (6.51), all of the Chern-Simons and Yang-Mills boundary terms vanish. Finally, the Yang-Mills action written in this way remains $Q$-exact with these boundary conditions, again without the need to add a compensating boundary action [58].

Given (6.51), one can choose a gauge in which the saddle points of the Yang-Mills action are given by $A_{\chi}=0, A_{\tilde{\mu}}=a_{\tilde{\mu}}, \sigma=\sigma_{0}$ where the constants $a_{\tilde{\mu}}$ and $\sigma_{0}$ satisfy $\left[a_{\tilde{\mu}}, \sigma_{0}\right]=0$. Moreover, regularity of the gauge field at $\chi=0$ requires $a_{\phi_{2}}=0$; as long as $\chi_{0}<\pi / 2, a_{\phi_{1}}$ can be nonzero, and it is only this component on which a localized Wilson loop depends. Namely, a BPS Wilson loop along a curve $\gamma$ at fixed $\chi \in\left[0, \chi_{0}\right]$ localizes as follows:

$$
W=\operatorname{Tr}_{R} P \exp \left[i \oint_{\gamma} d \tau\left(A_{\mu} \dot{x}^{\mu}-i \sigma|\dot{x}|\right)\right] \rightarrow\langle W\rangle=\operatorname{Tr}_{R} e^{2 \pi i \ell\left(a_{\phi_{1}}-i \sigma_{0}\right)} .
$$

For $\chi \in\left(0, \chi_{0}\right], \gamma$ is an unknot represented as a $(1,1)$ torus knot. At the core of the torus $(\chi=0), \gamma$ is a $(1,0)$ torus knot. For any $\chi, \gamma$ has length $2 \pi \ell$. Because the value of $\sigma$ at the saddle point is fixed rather than integrated over, the expectation value of a Wilson loop is trivial to compute: both the classical contribution and the one-loop determinants cancel in the normalized expectation value.

Note that $\chi$ parametrizes the time direction in Hamiltonian quantization on $T^{2}$. Hence we would like to interpret (6.53) (or rather, the corresponding unnormalized expectation value) as the wavefunction of a state in the Hilbert space on $T^{2}$. This interpretation is not straightforward. One obvious shortcoming is that with the half-BPS Dirichlet boundary conditions used for localization, one cannot obtain an arbitrary wavefunctional of the boundary fields from the path integral on the torus; rather, it is evaluated at particular boundary values of the gauge field. It is tempting to compare this result to that obtained from holomorphic (coherent state) quantization [21, 69]. There, one obtains affine (WeylKac) characters at level $k$. Recall that an affine character is a combination of a Virasoro character and an ordinary Weyl character: for $\widehat{G}_{k}$ with vacuum representation of highest weight $\lambda$,

$$
\chi_{\lambda}^{(k)}\left(\tau, \theta^{i}\right)=q^{-c / 24} \operatorname{tr}_{\lambda}^{(k)}\left(q^{L_{0}} e^{i \theta^{i} H^{i}}\right)
$$

where the $H^{i}$ are Cartan generators. However, to arrive at an answer that is a function (rather than a functional) of the constant $\phi_{1}$-component, we have effectively chosen a real polarization [21]; moreover, the localization calculation implicitly fixes a complex structure on the $T^{2}$ rather than allowing it to be arbitrary. Hence we cannot hope to reproduce the 
Kac-Moody part of the affine character, which depends on the modular parameter $\tau$; only the Weyl character for compact $G$ is visible in this calculation. What we can do is isolate the shift in the highest weight $\lambda$, because the Weyl character already fixes $\lambda$. This is enough for our purposes. The constant Cartan element in the supersymmetric boundary condition for the gauge field is identified with the Cartan parameter of the Hodge decomposition on the torus (D.12), which determines the Cartan angles that appear in the Weyl character. Note that after fixing a gauge, we must still add a boundary term to make the variational principle well-defined in our real polarization (see appendix D.2); this term vanishes on the localization locus because it involves the product of both components of the gauge field along the boundary torus.

To be slightly more precise, the wavefunctions (D.26) obtained via holomorphic quantization are functionals of $A_{z}$; restricting them to functions of $A_{z}=a_{z}$ where $a_{z}$ is a constant in the Cartan subalgebra shows that a basis for the physical wavefunctions is given by Weyl-Kac characters at level $k$, labeled by distinct $\lambda \in \Lambda_{W} /\left(\mathcal{W} \ltimes k \Lambda_{R}\right)$ :

$$
\psi_{\lambda}\left(a_{z}\right) \equiv e^{-\frac{k \operatorname{Im} \tau}{\pi} \operatorname{Tr} a_{z}^{2}} \chi_{\lambda}^{(k)}(\bar{\tau}, u), \quad u \equiv-\frac{\operatorname{Im} \tau}{\pi} a_{z}
$$

For constant $a_{z}$, one might naïvely make a change of variables to interpret the wavefunctions as functions of the coordinates $a_{1}$. However, it is the passage from holomorphic to real polarization that involves nontrivial Jacobians and leads to the famous shifts [21]. Instead of setting $A_{z}=a_{z}$, one should integrate out the modes that are not constant and in the Cartan to obtain an effective wavefunction for $a_{z}$, leading to an effective wavefunction in $a_{1}$ that coincides with the naïve one up to the famous shifts (see appendix D.4).

Localization on the solid torus $D^{2} \times S^{1}$ has previously been discussed in [59], but this discussion does not fit neatly into our framework for Seifert manifolds because it uses both a different metric and different boundary conditions than [58]. Nonetheless, it is also found in [59] that the $\mathcal{N}=2$ Yang-Mills action can be written in a $Q$-exact form without surface terms. On the other hand, the $\mathcal{N}=2$ Chern-Simons action is invariant under neither SUSY nor gauge transformations in the presence of a boundary. Two proposals are given in [59] for maintaining gauge invariance, namely that the compensating boundary action should contain either a chiral 2D $(0,2)$ theory or a trivially supersymmetric gauged chiral WZW model obtained by viewing gauge parameters as physical fields on the boundary. The second option makes direct contact with the Chern-Simons wavefunction computed in holomorphic polarization: the Kähler potential in the inner product (D.18) of coherent states appears from the localization point of view as a boundary term necessary to preserve half-BPS boundary conditions (again, the localization computation selects a constant gauge field, for which the wavefunction becomes a Weyl-Kac character). However, as in holomorphic quantization, one obtains a character in the representation $\lambda$ by taking Weyl-invariant linear combinations of generalized theta functions rather than by directly evaluating the path integral with a Wilson loop in the representation $\lambda$; it is not clear how such a Wilson loop would fit into the approach of [59]. ${ }^{22}$

\footnotetext{
${ }^{22}$ The computation of [59] can be viewed as that of a half-index with Neumann boundary conditions for the vector multiplet. For a discussion of how to recover Weyl-Kac characters from the half-index with Dirichlet $(0,2)$ boundary conditions in $\mathcal{N}=2$ Chern-Simons theories, see [70].
} 


\section{Matching $\mathcal{N}=0$ and $\mathcal{N}=2$ line operators}

So far, we have explained the quantum-mechanical non-renormalization of the weight only for certain classes of BPS observables in pure $\mathcal{N}=2$ Chern-Simons, which can be computed via localization on three-manifolds that admit a real, nowhere vanishing Killing vector. This amounts to an explanation of the renormalization of the weight for a similarly restricted set of observables in the corresponding $\mathcal{N}=0$ theory. The correspondence is as follows: embed the $\mathcal{N}=0$ theory in an $\mathcal{N}=2$ theory with the appropriate level ("integrate in" auxiliary fields), and then for those links that are deformable to a BPS configuration, deform them to said configuration and enrich them with $\sigma$ as in (6.16). This operation is trivial at the level of the functional integral. Clearly, this procedure is not possible for arbitrary links, even though all observables are explicitly computable by topological means. While we cannot explain the non-renormalization of the weight for completely general observables, we have sketched how one might approach a more general understanding by localizing on a solid torus. In this section, we make some further comments on the correspondence between $\mathcal{N}=0$ and $\mathcal{N}=2$ observables.

\section{1 (Non-)renormalization}

To substantiate the claim that the natural UV completion of Chern-Simons theory should have $\mathcal{N}=2$ supersymmetry, it is (as mentioned in the introduction) important to fix unambiguous definitions of the level $k$ and the representation $\lambda$. Throughout, we have used the canonical definition that the $k$ in $\mathcal{N}=0 G_{k}$ Chern-Simons theory is the level of the corresponding 2D WZW model, where it appears in correlation functions and has a precise physical meaning. Relative to this definition, the level that appears in the Chern-Simons braiding matrix with parameter $q$ is $k+h .^{23}$ This shift is independent of regularization scheme, i.e., the question of how the renormalized coupling depends on the bare coupling. Said differently, our $k \equiv k_{\text {phys }}$ is what determines the dimension of the Hilbert space and changes sign under time reversal, while $k_{\text {phys }}+h$ is what appears in correlation functions. The relation of $k_{\text {phys }}$ to some UV parameter $k_{\text {bare }}$ (e.g., via $k_{\text {phys }}=k_{\text {bare }}+h$ or $k_{\text {phys }}=k_{\text {bare }}$ ) is a question of regularization scheme and not physically meaningful.

On the other hand, $\lambda$ determines the conjugacy class of the holonomy around a Wilson loop to be $e^{-2 \pi i \lambda / k}$, as measured by another loop that links it. This relation, derived from the classical EOMs (see appendix D.3), receives quantum corrections. For example, in the case of $\mathrm{SU}(2)$ (and using our convention for $\lambda$ from section 4), the classical and quantum holonomies are $e^{2 \pi i j \sigma_{3} / k}$ and $e^{2 \pi i(j+1 / 2) \sigma_{3} /(k+2)}$, respectively. To interpret the statement that " $\lambda$ is not renormalized" in the $\mathcal{N}=2$ setting, it should be kept in mind that Wilson loops are typically written not in terms of the bare $\lambda$, but rather in terms of an effective $\lambda$ that corresponds to having integrated out the fermions along the line.

\subsection{D point of view}

For completeness, let us comment on how the differences between the bosonic and supersymmetric theories bear on the mapping of line operators between the two theories.

\footnotetext{
${ }^{23}$ Monodromy matrices in Chern-Simons theory follow from $R$-matrices in braid representations, and by "braiding matrix," we mean the half-monodromy matrix [68].
} 
An obvious difference is that the $\mathcal{N}=2$ theory contains extra bulk fields, as well as both supersymmetric and non-supersymmetric line operators. Wilson loops in $\mathcal{N}=0$ CS are functions of the gauge field $A$, while Wilson loops in $\mathcal{N}=2 \mathrm{CS}$ are (schematically) functions of the combination $A+\sigma$. A collection of the former loops in an arbitrary smooth configuration and a collection of the latter loops in the same configuration have identical correlation functions in the respective theories, up to an appropriate identification of parameters. This is true even if the configuration is not BPS from the point of view of the latter theory, hence not calculable using localization, as one sees by integrating out $\sigma$. Moreover, in the latter theory, correlation functions of non-intersecting loops not involving local operators constructed from the extra bulk fields are independent of whether the loop operators are written as functions of $A$ or of $A+\sigma$. However, such correlation functions can still have contact terms with integrated local operators; these contact terms differ for $A$ and $A+\sigma$ loops. The Schwinger-Dyson equation for $A$ says that both $\mathcal{N}=0$ and $\mathcal{N}=2$ loops have contact terms with the equation of motion for $A$. The Schwinger-Dyson equation for $\sigma$ says that only $\mathcal{N}=2$ loops have contact terms with the auxiliary scalar $D$ in the $\mathcal{N}=2$ vector multiplet. At finite Yang-Mills coupling, the $A$ and $\sigma$ EOMs involve fermionic sources, but these irrelevant terms are $Q$-exact, so do not affect correlation functions of BPS loops. If one is only interested in correlators of non-intersecting loops, as we are, then these issues are not relevant. For a related discussion of the loop equation for BPS Wilson loops, see section 4 of [71].

To properly define the localizing term requires both a metric and a spin structure (the latter because the fermions become dynamical at finite Yang-Mills coupling), neither of which seem to be necessary to define the bosonic theory [27]. ${ }^{24}$ But recall that a metric is already needed both to regularize and to gauge-fix the bosonic theory [10]. Moreover, recall that computing observables in $\mathcal{N}=0$ Chern-Simons requires choosing a framing of $M^{3}$, which automatically fixes a spin structure. Therefore, even at finite Yang-Mills coupling, the regularized pure $\mathcal{N}=2$ Chern-Simons theory does not depend on any additional geometric data beyond that required to compute observables in the $\mathcal{N}=0$ theory. ${ }^{25}$

\subsection{A quasi-2D point of view}

We now show that there exists a one-to-one correspondence between line operators in the bosonic and supersymmetric theories that is clear only if we take into account both shifts.

As can be seen in canonical quantization, the distinct Wilson lines in pure ChernSimons theory are in one-to-one correspondence with the ground states of the theory on a (spatial) torus. To explain what "distinct" means, we must identify the precise equivalence

\footnotetext{
${ }^{24}$ The Killing spinor equations (6.1) require only a $\operatorname{spin}^{c}$ structure, which exists on any orientable threemanifold [24].

${ }^{25}$ There are at least two qualifications to this statement. First, while the non-topological localizing terms are $Q$-exact, the metric still enters into the computation of observables in a more essential way in the $\mathcal{N}=2$ theory because BPS Wilson loops must lie along isometries. However, just as in the $\mathcal{N}=0$ theory, smoothly deforming links in the (non-manifestly topological) $\mathcal{N}=2$ theory leaves correlation functions unchanged (to see this, set the coefficient of the localizing term to zero and integrate out the extra bulk fields). Second, the localization procedure determines the framing of knots, which is additional data beyond the framing of the three-manifold (the latter cancels in normalized expectation values).
} 
classes of Wilson lines that map to these ground states. $\mathrm{SU}(2)_{k}$ Chern-Simons on a torus has $k+1$ ground states labeled by half-integers $j=0, \ldots, k / 2$. These can equivalently be viewed as the $k+1$ primary operators in the $\mathrm{SU}(2)_{k}$ WZW model. From the 3D point of view, however, a Wilson line can carry any spin $j$. To respect the $2 \mathrm{D}$ truncation, all such lines fall into equivalence classes labeled by the basic lines $j=0, \ldots, k / 2$. The equivalence relations turn out to be a combination of Weyl conjugation and translation [21]:

$$
j \sim-j, \quad j \sim j+k .
$$

As reviewed in appendix D.3, the story is similar for general $G$. Line operators are subject to equivalence relations given by the action of the affine Weyl group at level $k\left(\mathcal{W} \ltimes k \Lambda_{R}^{\vee}\right.$ where $\Lambda_{R}^{\vee}$ is the coroot lattice of $G$ ), whose fundamental domain we refer to as an affine Weyl chamber or a Weyl alcove and which contains all inequivalent weights (corresponding to integrable representations of $\left.\widehat{G}_{k}\right){ }^{26}$

Now consider the correlation functions of these lines. Two basic observables of SU(2) $k$ Chern-Simons on $S^{3}$ are the expectation value of an unknotted spin- $j$ Wilson loop and the expectation value of two Wilson loops of spins $j, j^{\prime}$ in a Hopf link:

$$
\left\langle W_{j}\right\rangle_{\mathcal{N}=0}=\frac{S_{0 j}}{S_{00}}, \quad\left\langle W_{j} W_{j^{\prime}}\right\rangle_{\mathcal{N}=0}=\frac{S_{j j^{\prime}}}{S_{00}}
$$

Recall that the modular $S$-matrix of $\mathrm{SU}(2)_{k}$ is given by (2.11) in a basis of integrable representations. The formulas (7.2) apply only to Wilson loops with $j$ within the restricted range $0, \ldots, k / 2$. Indeed, (2.11) is not invariant under the equivalence relations (7.1). Nonetheless, let us naïvely extend these formulas to arbitrary $j, j^{\prime}$. The first positive value of $j$ for which $\left\langle W_{j}\right\rangle=0$ is that immediately above the truncation threshold: $j=(k+1) / 2$. More generally, from (7.2), it is clear that a line of spin $j$ and a line of spin $j+k+2$ have identical correlation functions, while lines with $j=n(k / 2+1)-1 / 2$ for any integer $n$ vanish identically. Here, one should distinguish the trivial line $j=0$, which has $\left\langle W_{0}\right\rangle=1$ and trivial braiding with all other lines, from nonexistent lines, which have $\left\langle W_{j}\right\rangle=0$ and vanishing correlation functions with all other lines. On the other hand, a line with $j$ and a line with $j+k / 2+1$ have the same expectation value and braiding, up to a sign. In other words, at the level of correlation functions, $\mathrm{SU}(2)_{k}$ Wilson lines are antiperiodic with period $k / 2+1$.

An analogous antiperiodicity phenomenon holds for arbitrary simple, compact $G$. In the WZW model, the fusion rule eigenvalues (computed from the $S$-matrix elements) are equal to the finite Weyl characters of $G$, evaluated on some special Cartan angles that respect the truncation of the relevant representations [73]. For example, in $\mathrm{SU}(2)_{k}$, $\lambda_{\ell}^{(n)}=S_{\ell n} / S_{0 n}$ is the Weyl character $\chi_{\ell}(\theta)$ in $(1.2)$ evaluated at $\theta / 2=(2 n+1) \pi /(k+2)$ for $n=0, \ldots, k / 2$, chosen such that the Weyl character of $\operatorname{spin} \ell=(k+1) / 2$ vanishes.

The (anti)periodicity of $S$ under $j \rightarrow j+(k+2) / 2$ can be understood in terms of the renormalized parameters $K=k+2$ and $J=j+1 / 2 \cdot{ }^{27}$ In the $\mathcal{N}=2$ theory, a $J$ Wilson

\footnotetext{
${ }^{26}$ The equivalence classes of Wilson lines in abelian Chern-Simons can be found in appendix C of [72].

${ }^{27}$ See also [22]. I thank V. Mikhaylov for correspondence on this point.
} 
line has holonomy $e^{2 \pi i J \sigma_{3} / K}$, so the equivalence relations are

$$
J \sim-J, \quad J \sim J+K \quad \Longleftrightarrow \quad j \sim-1-j, \quad j \sim j+k+2 .
$$

The inequivalent values of $j$ are $-1 / 2, \ldots,(k+1) / 2$. The extremal values $j=-1 / 2$ and $j=(k+1) / 2$ correspond to identically zero line operators, and the remaining values are the same as in the $\mathcal{N}=0$ formulation. In other words, in contrast to (7.2) for $\mathcal{N}=0$ $\mathrm{SU}(2)_{k}$ on $S^{3}$, we have for $\mathcal{N}=2 \mathrm{SU}(2)_{K}$ on $S^{3}$ that

$$
\left\langle W_{J}\right\rangle_{\mathcal{N}=2}=\frac{S_{\frac{1}{2} J}}{S_{\frac{1}{2} \frac{1}{2}}}, \quad\left\langle W_{J} W_{J^{\prime}}\right\rangle_{\mathcal{N}=2}=\frac{S_{J J^{\prime}}}{S_{\frac{1}{2} \frac{1}{2}}}, \quad S_{J J^{\prime}} \equiv \sqrt{\frac{2}{K}} \sin \left[\frac{(2 J)\left(2 J^{\prime}\right) \pi}{K}\right],
$$

where the bare $J$ must satisfy $J \geq 1 / 2$ for supersymmetry not to be spontaneously broken. In the supersymmetric theory, labeling lines by $J=1 / 2, \ldots,(k+1) / 2$, the $J=0$ line does not exist due to the vanishing Grassmann integral over the zero modes of the fermion in the $\mathcal{N}=2$ coadjoint orbit sigma model. The conclusion is that the $\mathcal{N}=2$ theory has the same set of independent line operators as the $\mathcal{N}=0$ theory. In the $\mathcal{N}=2$ formulation, the $S$-matrix $S_{j j^{\prime}}$ is explicitly invariant under the equivalence relations (7.3).

For general $G$, let $\Lambda=\lambda+\rho$ and $K=k+h$. Then $\Lambda$, modulo the action of the affine Weyl group at level $K$, takes values in an affine Weyl chamber at level $K$. Those $\lambda=\Lambda-\rho$ for $\Lambda$ at the boundary of the chamber correspond to nonexistent lines, while those for $\Lambda$ in the interior are in one-to-one correspondence with weights in the affine Weyl chamber at level $k$ (for further details, see appendix D.4).

It would be interesting to understand both shifts from an intrinsically $2 \mathrm{D}$ point of view, namely to translate the equivalence between $(J, K)$ and $(j, k)$ into an equivalence between ordinary and super WZW models [74-76].

\section{Discussion}

Using $\mathrm{SU}(2)$ as a case study, we have supersymmetrized the coadjoint orbit quantum mechanics on a Wilson line in flat space from both intrinsically $1 \mathrm{D}$ and $3 \mathrm{D}$ points of view, providing several complementary ways to understand the shift in the representation $j$. We have described how to extend this understanding to certain compact Euclidean manifolds. For some classes of observables in Chern-Simons theory, the existence of an auxiliary supersymmetry lends itself not only to conceptual unity, but also to increased computability.

For arbitrary simple groups, one has both generic and degenerate coadjoint orbits, corresponding to quotienting $G$ by the maximal torus $T$ or by a Levi subgroup $L \supset T$ (see [77] and references therein). For example, the gauge group $\mathrm{SU}(N+1)$ has for a generic orbit the phase space $\mathrm{SU}(N+1) / \mathrm{U}(1)^{N}$, a flag manifold with real dimension $N^{2}+$ $N$ (corresponding to a regular weight); on the other hand, the most degenerate orbit is $\mathrm{SU}(N+1) /(\mathrm{SU}(N) \times \mathrm{U}(1)) \cong S^{2 N+1} / S^{1} \cong \mathbb{C P}^{N}$, which has $2 N$ real dimensions and a simple Kähler potential (corresponding to a weight that lies in the most symmetric part of the boundary of the positive Weyl chamber). The quantization of the phase space $\mathbb{C P}^{N}$ is 
well-known and can be made very explicit in terms of coherent states (see, e.g., section 5 of [8]). The Fubini-Study metric for $\mathbb{C P}^{N}$ follows from covering the manifold with $N+1$ patches with the Kähler potential in each patch being the obvious generalization of that for $\mathrm{SU}(2)$. In principle, one can carry out a similar analysis with $\mathrm{SU}(N+1)$ Killing vectors. We will not attempt the full analysis in this paper. We simply remark that in general, the shift of a fundamental weight by the Weyl vector is no longer a fundamental weight, so one would need a qualitatively different sigma model than the original to describe the coadjoint orbit of the shifted weight. An option is not to work in local coordinates at all, along the lines of [14] (however, this approach seems harder to supersymmetrize).

Finally, perhaps this story is more natural in a setting with twice as much supersymmetry $(3 \mathrm{D} \mathcal{N}=4)$, where one has the option of twisting spatial rotations by either of the $\mathrm{SU}(2) R$-symmetry groups, allowing for the construction of $1 / 4$-BPS Wilson or vortex loops supported on arbitrary curves [78].

\section{Acknowledgments}

I thank B. Le Floch, S. Giombi, I. Klebanov, V. Mikhaylov, S. Pufu, P. Putrov, N. Seiberg, H. Verlinde, B. Willett, E. Witten, and I. Yaakov for helpful discussions or correspondence. I would like to give a special thanks to N. Seiberg for suggesting this line of investigation. I am also grateful to M. Dedushenko, S. Pufu, and R. Yacoby for collaboration on related work. This work was supported in part by the NSF GRFP under Grant No. DGE-1656466 and by the Graduate School at Princeton University.

\section{A Conventions}

\section{A.1 Gauge transformations}

We use $\left[T^{a}, T^{b}\right]=i f^{a b c} T^{c}$ with $f^{a b c}$ real and $\operatorname{Tr}\left(T^{a} T^{b}\right)=\frac{1}{2} \delta^{a b}$. We have

$$
\mathcal{L}_{\mathrm{CS}}^{g}=\epsilon^{\mu \nu \rho} \operatorname{Tr}\left(A_{\mu}^{g} \partial_{\nu} A_{\rho}^{g}-\frac{2 i}{3} A_{\mu}^{g} A_{\nu}^{g} A_{\rho}^{g}\right), \quad \mathcal{L}_{\mathrm{CS}} \equiv \mathcal{L}_{\mathrm{CS}}^{\mathrm{id}}
$$

Writing $g=e^{i \theta^{a} T^{a}}$, we have $A_{\mu}^{g}=g A_{\mu} g^{-1}-i \partial_{\mu} g g^{-1}$ and

$$
\mathcal{L}_{\mathrm{CS}}^{g}=\mathcal{L}_{\mathrm{CS}}-i \epsilon^{\mu \nu \rho} \partial_{\mu} \operatorname{Tr}\left(A_{\nu} g^{-1} \partial_{\rho} g\right)-\frac{1}{3} \epsilon^{\mu \nu \rho} \operatorname{Tr}\left[\left(\partial_{\mu} g g^{-1}\right)\left(\partial_{\nu} g g^{-1}\right)\left(\partial_{\rho} g g^{-1}\right)\right] .
$$

In coordinate-free notation, $A^{g}=g A g^{-1}-i d g g^{-1}$ and

$$
\operatorname{Tr}\left[A^{g} d A^{g}-\frac{2 i}{3}\left(A^{g}\right)^{3}\right]=\operatorname{Tr}\left[A d A-\frac{2 i}{3} A^{3}-i d\left(A g^{-1} d g\right)-\frac{1}{3}\left(d g g^{-1}\right)^{3}\right] .
$$

\section{A.2 $1 \mathrm{D} \mathcal{N}=2$}

We work in Lorentzian ${ }^{28} 1 \mathrm{D} \mathcal{N}=2$ superspace with coordinates $\left(t, \theta, \theta^{\dagger}\right)$ and let $\epsilon$ be a complex spinor parameter. The representations of the supercharges as differential operators

\footnotetext{
${ }^{28}$ To Euclideanize the following, take $\tau=i t$ and $i S=-S_{E}$.
} 
on superspace and the supercovariant derivatives are

$$
\begin{array}{ll}
\hat{Q}=\partial_{\theta}+i \theta^{\dagger} \partial_{t}, & \hat{Q}^{\dagger}=\partial_{\theta^{\dagger}}+i \theta \partial_{t}, \\
D=\partial_{\theta}-i \theta^{\dagger} \partial_{t}, & D^{\dagger}=\partial_{\theta^{\dagger}}-i \theta \partial_{t} .
\end{array}
$$

The nonvanishing anticommutators are

$$
\left\{\hat{Q}, \hat{Q}^{\dagger}\right\}=-\left\{D, D^{\dagger}\right\}=2 i \partial_{t}
$$

A general superfield takes the form

$$
\Xi(t, \theta)=\phi(t)+\theta \psi(t)+\theta^{\dagger} \chi(t)+\theta \theta^{\dagger} F(t)
$$

with SUSY acting as $\delta \Xi=\left(\epsilon \hat{Q}+\epsilon^{\dagger} \hat{Q}^{\dagger}\right) \Xi$, or in components as

$$
\begin{aligned}
\delta \phi & =\epsilon \psi+\epsilon^{\dagger} \chi, \\
\delta \psi & =-i \epsilon^{\dagger} \dot{\phi}+\epsilon^{\dagger} F, \\
\delta \chi & =-i \epsilon \dot{\phi}-\epsilon F, \\
\delta F & =-i \epsilon \dot{\psi}+i \epsilon^{\dagger} \dot{\chi}
\end{aligned}
$$

All components of $\Xi$ are complex. The vector multiplet satisfies

$$
V=V^{\dagger} \Longrightarrow V(t, \theta)=\phi(t)+\theta \psi(t)-\theta^{\dagger} \psi^{\dagger}(t)+\theta \theta^{\dagger} F(t) .
$$

The SUSY transformations of its components $\phi$ (real), $\psi$ (complex), and $F$ (real) are

$$
\begin{aligned}
\delta \phi & =\epsilon \psi-\epsilon^{\dagger} \psi^{\dagger}, \\
\delta \psi & =-i \epsilon^{\dagger} \dot{\phi}+\epsilon^{\dagger} F, \\
\delta \psi^{\dagger} & =i \epsilon \dot{\phi}+\epsilon F, \\
\delta F & =-i \epsilon \dot{\psi}-i \epsilon^{\dagger} \dot{\psi}^{\dagger} .
\end{aligned}
$$

Here, $F$ plays the role of the non-dynamical gauge field, so that the 1D Chern-Simons action $\int F$ is automatically both gauge- and SUSY-invariant. The chiral multiplet satisfies

$$
D^{\dagger} \Phi=0 \Longrightarrow \Phi(t, \theta)=\phi(t)+\theta \psi(t)-i \theta \theta^{\dagger} \dot{\phi}(t) .
$$

The SUSY transformations of its components $\phi$ (complex) and $\psi$ (complex) are

$$
\begin{aligned}
\delta \phi & =\epsilon \psi, \\
\delta \psi & =-2 i \epsilon^{\dagger} \dot{\phi} .
\end{aligned}
$$

To integrate over superspace, we use $d^{2} \theta \equiv d \theta^{\dagger} d \theta$.

The 1D SUSY' transformations are derived as follows. In Wess-Zumino gauge, we have $\left.V\right|_{\mathrm{WZ}}=\theta \theta^{\dagger} F$, which transforms under SUSY to $V^{\prime}=\left.V\right|_{\mathrm{WZ}}+\left.\delta V\right|_{\mathrm{WZ}}$. To preserve Wess-Zumino gauge, we choose the compensatory super gauge transformation parameter $\Phi=i \Lambda$ such that $\left.\delta V\right|_{\mathrm{WZ}}+\frac{1}{2}\left(\Phi+\Phi^{\dagger}\right)$ is $O\left(\theta \theta^{\dagger}\right)$ :

$$
\left.e^{2 V^{\prime}} \rightarrow e^{\Phi} e^{2 V^{\prime}} e^{\Phi^{\dagger}} \Longleftrightarrow V^{\prime} \rightarrow V\right|_{\mathrm{WZ}},
$$


which means that $\delta^{\prime}$ acts trivially on the vector multiplet. For the chiral multiplet, only the transformation rule for $\psi$ is modified by taking $\partial_{0} \rightarrow D_{0}$ :

$$
\begin{aligned}
\delta^{\prime} \phi & =\epsilon \psi, \\
\delta^{\prime} \psi & =-2 i \epsilon^{\dagger} D_{0} \phi,
\end{aligned}
$$

where the gauge field appearing in $D_{0}$ is the single nonzero component of $\left.V\right|_{\text {wz }}$.

\section{A.3 $3 \mathrm{D} \mathcal{N}=2$}

We raise and lower spinor indices on the left by $\epsilon^{\alpha \beta}=-\epsilon_{\alpha \beta}\left(\epsilon^{12} \equiv 1\right)$, with $\psi \chi \equiv \epsilon^{\alpha \beta} \psi_{\alpha} \chi_{\beta}$ (cf. [79]). This convention requires that we distinguish matrix multiplication (".") from spinor contraction (no symbol), which differ by a sign:

$$
\left(\gamma^{a} \xi\right)_{\alpha}=-\left(\gamma^{a}\right)_{\alpha}{ }^{\beta} \xi_{\beta} \Longleftrightarrow \gamma^{a} \xi=-\gamma^{a} \cdot \xi^{29}
$$

The basic Fierz identities, written with a free spinor index (taken to be lower by default), take the same form for both commuting and anticommuting spinors:

$$
\xi(\zeta \lambda)+\zeta(\lambda \xi)+\lambda(\xi \zeta)=0, \quad 2 \lambda(\xi \zeta)+\xi(\zeta \lambda)+\gamma^{\mu} \xi\left(\zeta \gamma_{\mu} \lambda\right)=0
$$

By default, our spinors are anticommuting; the notation "| 0 " applied to a Grassmann-odd spinor denotes its Grassmann-even version. Spinors satisfy $\psi \chi= \pm \chi \psi, \psi \gamma^{\mu} \chi=\mp \chi \gamma^{\mu} \psi$, $\overline{\psi \chi}=\mp \bar{\psi} \bar{\chi}$ depending on whether they anticommute (top sign) or commute (bottom sign).

Spinors that would be conjugate (e.g., $\lambda, \bar{\lambda}$ ) in Lorentzian signature are independent in Euclidean signature (e.g., $\lambda, \tilde{\lambda}$ ). Lower and upper indices denote the fundamental and antifundamental, respectively. In Lorentzian signature, as in $1 \mathrm{D}$, we use $x^{*}, \bar{x}, x^{\dagger}$ interchangeably to denote the complex conjugate of $x$. In Euclidean signature, we use bars and stars interchangeably to denote complex conjugation, while daggers denote Hermitian conjugation: $\left(\psi^{\dagger}\right)^{\alpha}=\left(\psi_{\alpha}\right)^{*}$ (the combination $\xi^{\dagger} \psi$ is $\mathrm{SU}(2)$-invariant).

\section{A.3.1 Lorentzian}

In $\mathbb{R}^{1,2}$ (with signature -++ ), the $3 \mathrm{D}$ gamma matrices are

$$
\left(\gamma^{\mu=0,1,2}\right)_{\alpha \beta}=\left(-1, \sigma^{1}, \sigma^{3}\right)_{\alpha \beta}, \quad \epsilon^{\alpha \beta} \gamma_{\gamma \alpha}^{\mu} \gamma_{\beta \delta}^{\nu}=\eta^{\mu \nu} \epsilon_{\gamma \delta}-\epsilon^{\mu \nu \rho}\left(\gamma_{\rho}\right)_{\gamma \delta} .
$$

With lowered indices, these matrices are real and symmetric, so that $\theta \gamma^{\mu} \bar{\theta}$ is real. We take $\epsilon^{012}=1$; note that the identity $\epsilon^{\mu \nu \lambda} \epsilon_{\lambda}^{\rho \sigma}=\eta^{\mu \sigma} \eta^{\nu \rho}-\eta^{\mu \rho} \eta^{\nu \sigma}$ differs by a sign relative to the Euclidean case. The $3 \mathrm{D} \mathcal{N}=2$ algebra is

$$
\left\{Q_{\alpha}, \bar{Q}_{\beta}\right\}=2 \gamma_{\alpha \beta}^{\mu} P_{\mu}+2 i \epsilon_{\alpha \beta} Z, \quad\left\{Q_{\alpha}, Q_{\beta}\right\}=0 .
$$

The representations of the supercharges as differential operators on superspace and the supercovariant derivatives are

$$
\begin{array}{ll}
\mathcal{Q}_{\alpha}=\frac{\partial}{\partial \theta^{\alpha}}-i \gamma_{\alpha \beta}^{\mu} \bar{\theta}^{\beta} \partial_{\mu}, & \overline{\mathcal{Q}}_{\alpha}=-\frac{\partial}{\partial \bar{\theta}^{\alpha}}+i \theta^{\beta} \gamma_{\beta \alpha}^{\mu} \partial_{\mu}, \\
D_{\alpha}=\frac{\partial}{\partial \theta^{\alpha}}+i \gamma_{\alpha \beta}^{\mu} \bar{\theta}^{\beta} \partial_{\mu}, & \bar{D}_{\alpha}=-\frac{\partial}{\partial \bar{\theta}^{\alpha}}-i \theta^{\beta} \gamma_{\beta \alpha}^{\mu} \partial_{\mu} .
\end{array}
$$

We abbreviate $\bar{\partial}_{\alpha} \equiv \partial / \partial \bar{\theta}^{\alpha}, \partial_{\beta} \equiv \partial / \partial \theta^{\beta}$ and define $\int d^{4} \theta \theta^{2} \bar{\theta}^{2}=1$.

\footnotetext{
${ }^{29}$ We also sometimes use "." to denote multiplication in the appropriate representation of the gauge group: for example, $\left[D_{\mu}, D_{\nu}\right](\cdots)=-i F_{\mu \nu} \cdot(\cdots)$.
} 
In component form, the SUSY transformations of the $3 \mathrm{D} \mathcal{N}=2$ vector and chiral multiplets (5.2) and (5.3) are

$$
\begin{aligned}
\delta C & =-(\xi \chi-\bar{\xi} \bar{\chi}), \\
\delta \chi_{\alpha}=\overline{\delta \bar{\chi}_{\alpha}} & =i \bar{\xi}_{\alpha} \sigma+\gamma_{\alpha \beta}^{\mu} \bar{\xi}^{\beta}\left(A_{\mu}+i \partial_{\mu} C\right)-\xi_{\alpha}(M+i N), \\
\delta(M+i N)=\overline{\delta(M-i N)} & =-2 i\left(\bar{\xi} \bar{\lambda}-\bar{\xi} \gamma^{\mu} \partial_{\mu} \chi\right), \\
\delta \sigma & =-(\xi \bar{\lambda}-\bar{\xi} \lambda), \\
\delta A_{\mu} & =i\left(\xi \gamma_{\mu} \bar{\lambda}+\bar{\xi} \gamma_{\mu} \lambda-\xi \partial_{\mu} \chi-\bar{\xi} \partial_{\mu} \bar{\chi}\right), \\
\delta \lambda_{\alpha}=\overline{\delta \bar{\lambda}_{\alpha}} & =-i \xi_{\alpha} D-i \gamma_{\alpha \beta}^{\mu} \xi^{\beta} \partial_{\mu} \sigma-\epsilon^{\mu \nu \rho}\left(\gamma_{\rho}\right)_{\alpha \beta} \xi^{\beta} \partial_{\mu} A_{\nu}, \\
\delta D & =-\left(\xi \gamma^{\mu} \partial_{\mu} \bar{\lambda}-\bar{\xi} \gamma^{\mu} \partial_{\mu} \lambda\right)
\end{aligned}
$$

(following from $\left.\delta_{\xi} V=(\xi \mathcal{Q}-\bar{\xi} \overline{\mathcal{Q}}) V\right)$ and

$$
\begin{aligned}
\delta A & =-\sqrt{2} \xi \psi, \\
\delta \psi_{\alpha} & =-\sqrt{2} \xi_{\alpha} F+i \sqrt{2} \gamma_{\alpha \beta}^{\mu} \bar{\xi}^{\beta} \partial_{\mu} A, \\
\delta F & =i \sqrt{2} \bar{\xi} \gamma^{\mu} \partial_{\mu} \psi
\end{aligned}
$$

(following from $\left.\delta_{\xi} \Phi=(\xi \mathcal{Q}-\bar{\xi} \overline{\mathcal{Q}}) \Phi\right)$. The 3D SUSY' transformations are derived as follows. Under SUSY, $\left.V\right|_{\mathrm{WZ}}$ transforms into $\left.V^{\prime} \equiv V\right|_{\mathrm{WZ}}+\left.\delta V\right|_{\mathrm{WZ}}$. To preserve Wess-Zumino gauge, we choose $\Lambda$ such that $\left.\delta V\right|_{\mathrm{wZ}}+\frac{1}{2}(\Lambda+\bar{\Lambda})$ is $O(\theta \bar{\theta})$ and set the lowest component of $\Lambda$ (the parameter of ordinary gauge transformations) to zero. With these choices, and to first order in $\xi, \bar{\xi}$, the super gauge transformation $e^{2 V^{\prime}} \rightarrow e^{\bar{\Lambda}} e^{2 V^{\prime}} e^{\Lambda}$ truncates to

$$
V^{\prime} \rightarrow V^{\prime}+\frac{1}{2}(\Lambda+\bar{\Lambda})+\frac{1}{2}\left[V^{\prime}, \Lambda-\bar{\Lambda}\right],
$$

from which we read off (5.9). SUSY' also modifies the chiral multiplet transformation laws by terms involving vector multiplet fields, so that $\Phi+\delta \Phi \rightarrow e^{-\Lambda}(\Phi+\delta \Phi)$ and $\bar{\Phi}+\delta \bar{\Phi} \rightarrow$ $(\bar{\Phi}+\delta \bar{\Phi}) e^{-\bar{\Lambda}}$, from which (5.10) follows.

\section{A.3.2 Euclidean}

In $\mathbb{R}^{3}$, we have

$$
\left(\gamma^{\mu=0,1,2}\right)_{\alpha \beta}=\left(-i, \sigma^{1}, \sigma^{3}\right)_{\alpha \beta}, \quad \epsilon^{\alpha \beta} \gamma_{\gamma \alpha}^{\mu} \gamma_{\beta \delta}^{\nu}=\delta^{\mu \nu} \epsilon_{\gamma \delta}-i \epsilon^{\mu \nu \rho}\left(\gamma_{\rho}\right)_{\gamma \delta} .
$$

In particular, with lowered indices, the gamma matrices are no longer real. We often write $\mu=1,2,3$ in place of $\mu=0,1,2$ in Euclidean signature.

The Euclidean SUSY' transformations of a vector multiplet are

$$
\begin{aligned}
\delta^{\prime} \sigma & =-(\xi \tilde{\lambda}-\tilde{\xi} \lambda), \\
\delta^{\prime} A_{\mu} & =i\left(\xi \gamma_{\mu} \tilde{\lambda}+\tilde{\xi} \gamma_{\mu} \lambda\right), \\
\delta^{\prime} \lambda & =-i \xi D-i \gamma^{\mu} \xi D_{\mu} \sigma-\frac{i}{2} \epsilon^{\mu \nu \rho} \gamma_{\rho} \xi F_{\mu \nu}, \\
\delta^{\prime} \tilde{\lambda} & =i \tilde{\xi} D+i \gamma^{\mu} \tilde{\xi} D_{\mu} \sigma-\frac{i}{2} \epsilon^{\mu \nu \rho} \gamma_{\rho} \tilde{\xi} F_{\mu \nu}, \\
\delta^{\prime} D & =-\left(\xi \gamma^{\mu} D_{\mu} \tilde{\lambda}-\tilde{\xi} \gamma^{\mu} D_{\mu} \lambda\right)+[\xi \tilde{\lambda}+\tilde{\xi} \lambda, \sigma]
\end{aligned}
$$


(compare to (5.9)), and those of a fundamental chiral multiplet are

$$
\begin{aligned}
& \delta^{\prime} A=-\sqrt{2} \xi \psi \\
& \delta^{\prime} \psi=-\sqrt{2} \xi F+i \sqrt{2} \gamma^{\mu} \tilde{\xi} D_{\mu} A+i \sqrt{2} \tilde{\xi} \sigma A \\
& \delta^{\prime} F=i \sqrt{2} \tilde{\xi} \gamma^{\mu} D_{\mu} \psi-i \sqrt{2} \sigma \tilde{\xi} \psi-2 i \tilde{\xi} \tilde{\lambda} A
\end{aligned}
$$

(compare to (5.10)). Writing $\delta^{\prime}=\delta_{\xi}^{\prime}+\delta_{\tilde{\xi}}^{\prime}$ with $\left(\delta_{\xi}^{\prime}\right)^{2}=\left(\delta_{\tilde{\xi}}^{\prime}\right)^{2}=0$, the SUSY' algebra is

$$
\left[\delta_{\xi_{1}}^{\prime}, \delta_{\xi_{2}}^{\prime}\right](\cdot)=\left[\delta_{\tilde{\xi}_{1}}^{\prime}, \delta_{\tilde{\xi}_{2}}^{\prime}\right](\cdot)=0, \quad\left[\delta_{\xi}^{\prime}, \delta_{\tilde{\xi}}^{\prime}\right](\cdot)=2 i \xi \gamma^{\mu} \tilde{\xi} D_{\mu}(\cdot)+2 i \xi \tilde{\xi} \sigma \cdot(\cdot)
$$

The Lagrangians of interest are

$$
\begin{aligned}
\left.\mathcal{L}_{\mathrm{CS}}\right|_{\mathbb{R}^{3}} & =\frac{k}{4 \pi i} \operatorname{Tr}\left[\epsilon^{\mu \nu \rho}\left(A_{\mu} \partial_{\nu} A_{\rho}-\frac{2 i}{3} A_{\mu} A_{\nu} A_{\rho}\right)-2 \lambda \tilde{\lambda}+2 i D \sigma\right], \\
\left.\mathcal{L}_{\mathrm{YM}}\right|_{\mathbb{R}^{3}} & =\frac{1}{g^{2}} \operatorname{Tr}\left(\frac{1}{4} F_{\mu \nu} F^{\mu \nu}+\frac{1}{2} D_{\mu} \sigma D^{\mu} \sigma-\frac{1}{2} D^{2}-i \tilde{\lambda} \gamma^{\mu} D_{\mu} \lambda+i \tilde{\lambda}[\sigma, \lambda]\right),
\end{aligned}
$$

where the path integration contour is over purely imaginary $D$. We find that

$$
\frac{1}{g^{2}} \delta_{\xi}^{\prime} \delta_{\tilde{\xi}}^{\prime} \operatorname{Tr}\left(\frac{1}{2} \lambda \tilde{\lambda}-i D \sigma\right)=\left.\xi \tilde{\xi} \mathcal{L}_{\mathrm{YM}}\right|_{\mathbb{R}^{3}}
$$

The Euclidean Yang-Mills action is in fact both $Q$-exact and $\tilde{Q}$-exact because in this case, the commutator of $\delta_{\xi}^{\prime}, \delta_{\tilde{\xi}}^{\prime}$ in (A.27) is the sum of a total derivative and a commutator, the latter of which vanishes inside the trace.

In curved Euclidean space, making appropriate modifications by the metric gives, e.g.,

$$
\epsilon^{\alpha \beta} \gamma_{\gamma \alpha}^{\mu} \gamma_{\beta \delta}^{\nu}=g^{\mu \nu} \epsilon_{\gamma \delta}-i \sqrt{g}^{-1} \epsilon^{\mu \nu \rho}\left(\gamma_{\rho}\right)_{\gamma \delta}
$$

( $\epsilon^{\mu \nu \rho}$ always denotes the Levi-Civita symbol; we make the corresponding tensor explicit by writing $\sqrt{g}^{-1} \epsilon^{\mu \nu \rho}$ or $\sqrt{g} \epsilon_{\mu \nu \rho}$ ). One can construct the superconformal algebra on a curved space that admits a conformal Killing spinor by diffeomorphism- and Weyl-covariantizing the corresponding flat-space Poincaré supersymmetry (or SUSY') algebra, in addition to replacing the constant supersymmetry parameters by conformal Killing spinors [80]. The conformal Killing spinor condition is

$$
\nabla_{\mu} \xi=\gamma_{\mu} \xi^{\prime} \Longleftrightarrow \xi^{\prime}=\frac{1}{3} \gamma^{\mu} \nabla_{\mu} \xi
$$

The subalgebra of the superconformal algebra that generates only isometries of the curved space is the curved-space analogue of the flat-space super-Poincaré algebra. On spinors,

$$
\nabla_{\mu} \psi=\left(\partial_{\mu}+\omega_{\mu}^{a b} \sigma_{a b}\right) \psi
$$

with $\sigma^{a b}=\frac{1}{8}\left[\gamma^{a}, \gamma^{b}\right]$. The Weyl weight $w(\varphi)$ of a field is the charge appearing in $\varphi \rightarrow$ $e^{-w \Omega} \varphi$. Our convention is that tensors with lower coordinate indices have zero Weyl weight (with raised indices, they gain a Weyl weight due to the metric) while tensors with tangent space indices generally have nonzero Weyl weight, with the difference being due 
to the transformation of the vielbein. The opposite is true for the metric, which is not a dynamical field: Weyl transformations act nontrivially on $g_{\mu \nu}$ and leave the tangent space metric unchanged. Thus for a scalar $\varphi$ and fermion $\psi$ of Weyl weight $w$,

$$
\begin{aligned}
\partial_{\mu} \varphi & \rightarrow e^{-w \Omega}\left(\partial_{\mu}-w \partial_{\mu} \Omega\right) \varphi \\
\gamma^{\mu} \nabla_{\mu} \psi & \rightarrow e^{-(w+1) \Omega} \gamma^{\mu} \nabla_{\mu} \psi+e^{-(w+1) \Omega}\left(\frac{d-1}{2}-w\right) \gamma^{\mu} \partial_{\mu} \Omega \psi .
\end{aligned}
$$

Note that $w\left(\gamma_{\mu}\right)=-1$ and $w\left(g_{\mu \nu}\right)=-2($ hence $w(\sqrt{g})=-d)$.

For the vector multiplet, our conventions fix the following Weyl weights:

$$
w(\sigma)=1, w\left(A_{\mu}\right)=0, w(\xi)=w(\tilde{\xi})=-1 / 2, w(\lambda)=w(\tilde{\lambda})=3 / 2, w(D)=2 .
$$

Aside from $A_{\mu}$ (which has $\left[A_{\mu}\right]=1$ ), these coincide with the scaling dimensions. In $d=3$, we have from (A.34) and (A.35) that

$$
w\left(\gamma^{\mu} \xi D_{\mu} \sigma+\frac{2}{3} \sigma \gamma^{\mu} \nabla_{\mu} \xi\right)=\frac{3}{2}, \quad w\left(\xi \gamma^{\mu} D_{\mu} \tilde{\lambda}-\frac{1}{3} \tilde{\lambda} \gamma^{\mu} \nabla_{\mu} \xi\right)=2
$$

and similarly for $(\xi, \tilde{\lambda}) \leftrightarrow(\tilde{\xi}, \lambda)$. To Weyl-covariantize (A.25), we replace the terms $\gamma^{\mu} \xi D_{\mu} \sigma$ and $\xi \gamma^{\mu} D_{\mu} \tilde{\lambda}$ with the expressions of well-defined Weyl weight in (A.36) (and similarly for $(\xi, \tilde{\lambda}) \leftrightarrow(\tilde{\xi}, \lambda)$ ), leading to the curved-space SUSY' transformations for a vector multiplet:

$$
\begin{aligned}
\delta^{\prime} \sigma & =-(\xi \tilde{\lambda}-\tilde{\xi} \lambda), \\
\delta^{\prime} A_{\mu} & =i\left(\xi \gamma_{\mu} \tilde{\lambda}+\tilde{\xi} \gamma_{\mu} \lambda\right), \\
\delta^{\prime} \lambda & =-i \xi D-i \gamma^{\mu} \xi D_{\mu} \sigma-\frac{i}{2} \sqrt{g}^{-1} \epsilon^{\mu \nu \rho} \gamma_{\rho} \xi F_{\mu \nu}-\frac{2 i}{3} \sigma \gamma^{\mu} \nabla_{\mu} \xi \\
\delta^{\prime} \tilde{\lambda} & =i \tilde{\xi} D+i \gamma^{\mu} \tilde{\xi} D_{\mu} \sigma-\frac{i}{2} \sqrt{g}^{-1} \epsilon^{\mu \nu \rho} \gamma_{\rho} \tilde{\xi} F_{\mu \nu}+\frac{2 i}{3} \sigma \gamma^{\mu} \nabla_{\mu} \tilde{\xi}, \\
\delta^{\prime} D & =-\left(\xi \gamma^{\mu} D_{\mu} \tilde{\lambda}-\tilde{\xi} \gamma^{\mu} D_{\mu} \lambda\right)+[\xi \tilde{\lambda}+\tilde{\xi} \lambda, \sigma]+\frac{1}{3}\left(\tilde{\lambda} \gamma^{\mu} \nabla_{\mu} \xi-\lambda \gamma^{\mu} \nabla_{\mu} \tilde{\xi}\right)
\end{aligned}
$$

where $D_{\mu}(\cdot)=\nabla_{\mu}(\cdot)-i\left[A_{\mu},(\cdot)\right]$ and $F_{\mu \nu}=\partial_{\mu} A_{\nu}-\partial_{\nu} A_{\mu}-i\left[A_{\mu}, A_{\nu}\right]$. Now consider a chiral multiplet with non-canonical scaling dimensions

$$
([A],[\psi],[F])=(\Delta, \Delta+1 / 2, \Delta+1),
$$

which coincide with the Weyl weights. In $d=3$, we have from (A.34) and (A.35) that

$$
w\left(\gamma^{\mu} \tilde{\xi} D_{\mu} A+\frac{2 \Delta}{3} A \gamma^{\mu} \nabla_{\mu} \tilde{\xi}\right)=\Delta+\frac{1}{2}, \quad w\left(\tilde{\xi} \gamma^{\mu} D_{\mu} \psi-\frac{2 \Delta-1}{3} \psi \gamma^{\mu} \nabla_{\mu} \tilde{\xi}\right)=\Delta+1 .
$$

To Weyl-covariantize (A.26), we replace $\gamma^{\mu} \tilde{\xi} D_{\mu} A$ and $\tilde{\xi} \gamma^{\mu} D_{\mu} \psi$ with the expressions in (A.38), leading to the curved-space SUSY' transformations for a fundamental chiral multiplet:

$$
\begin{aligned}
& \delta^{\prime} A=-\sqrt{2} \xi \psi, \\
& \delta^{\prime} \psi=-\sqrt{2} \xi F+i \sqrt{2}\left(\gamma^{\mu} \tilde{\xi} D_{\mu} A+\frac{2 \Delta}{3} A \gamma^{\mu} \nabla_{\mu} \tilde{\xi}\right)+i \sqrt{2} \tilde{\xi} \sigma A, \\
& \delta^{\prime} F=i \sqrt{2}\left(\tilde{\xi} \gamma^{\mu} D_{\mu} \psi-\frac{2 \Delta-1}{3} \psi \gamma^{\mu} \nabla_{\mu} \tilde{\xi}\right)-i \sqrt{2} \sigma \tilde{\xi} \psi-2 i \tilde{\xi} \tilde{\lambda} A
\end{aligned}
$$

where $D_{\mu}(\cdot)=\nabla_{\mu}(\cdot)-i A_{\mu}(\cdot)$. 
Closure of the 3D $\mathcal{N}=2$ superconformal algebra (i.e., the curved-space SUSY' algebra with conformal Killing spinor parameters) on the auxiliary fields requires a refinement of the conformal Killing spinor condition [50, 51, 81], namely that $\xi, \tilde{\xi}$ satisfy both (A.32) and

$$
\gamma^{\mu} \gamma^{\nu} \nabla_{\mu} \nabla_{\nu} \xi=h \xi \Longleftrightarrow 3 \gamma^{\mu} \nabla_{\mu} \xi^{\prime}=h \xi
$$

for some scalar function $h$. Using $\left[\nabla_{\mu}, \nabla_{\nu}\right] \psi=R_{\mu \nu}{ }^{a b} \sigma_{a b} \psi$ on fermions and $R_{\mu \nu \rho \sigma} \gamma^{\mu} \gamma^{\nu} \gamma^{\rho} \gamma^{\sigma}$ $=-2 R$, we deduce that $h=-3 R / 8$. Explicitly, the algebra is as follows. For $\xi, \tilde{\xi}$ satisfying (A.32), define the parameters

$$
\begin{aligned}
U^{\mu} & \equiv 2 i \xi \gamma^{\mu} \tilde{\xi}, & \epsilon_{\mu \nu} & \equiv 2 \sqrt{g} \epsilon_{\mu \nu \rho}\left(\tilde{\xi} \nabla^{\rho} \xi-\xi \nabla^{\rho} \tilde{\xi}\right), \\
\rho & \equiv \frac{2 i}{3} \nabla_{\mu}\left(\xi \gamma^{\mu} \tilde{\xi}\right), & \alpha & \equiv \frac{2 i}{3}\left(\nabla_{\mu} \xi \gamma^{\mu} \tilde{\xi}-\xi \gamma^{\mu} \nabla_{\mu} \tilde{\xi}\right) .
\end{aligned}
$$

For the vector multiplet, $\left[\delta_{\xi_{1}}^{\prime}, \delta_{\xi_{2}}^{\prime}\right]=\left[\delta_{\tilde{\xi}_{1}}^{\prime}, \delta_{\tilde{\xi}_{2}}^{\prime}\right]=0$ on all fields and

$$
\begin{aligned}
{\left[\delta_{\xi}^{\prime}, \delta_{\tilde{\xi}}^{\prime}\right] \sigma } & =U^{\mu} D_{\mu} \sigma+\rho \sigma \\
{\left[\delta_{\xi}^{\prime}, \delta_{\tilde{\xi}}^{\prime}\right] A_{\mu} } & =2 D_{\mu}(\xi \tilde{\xi} \sigma)+U^{\nu} F_{\nu \mu}, \\
{\left[\delta_{\xi}^{\prime}, \delta_{\tilde{\xi}}^{\prime}\right] F_{\mu \nu} } & =U^{\rho} D_{\rho} F_{\mu \nu}+2 i \xi \tilde{\xi}\left[\sigma, F_{\mu \nu}\right]+\epsilon_{\mu \rho} F^{\rho}{ }_{\nu}-\epsilon_{\nu \rho} F^{\rho}{ }_{\mu}+2 \rho F_{\mu \nu}, \\
{\left[\delta_{\xi}^{\prime}, \delta_{\tilde{\xi}}^{\prime}\right] \lambda } & =U^{\mu} D_{\mu} \lambda+2 i \xi \tilde{\xi}[\sigma, \lambda]+\epsilon_{\mu \nu} \sigma^{\mu \nu} \lambda+\frac{3}{2} \rho \lambda-\alpha \lambda \\
{\left[\delta_{\xi}^{\prime}, \delta_{\tilde{\xi}}^{\prime} \tilde{\lambda}\right.} & =U^{\mu} D_{\mu} \tilde{\lambda}+2 i \xi \tilde{\xi}[\sigma, \tilde{\lambda}]+\epsilon_{\mu \nu} \sigma^{\mu \nu} \tilde{\lambda}+\frac{3}{2} \rho \tilde{\lambda}+\alpha \tilde{\lambda} \\
{\left[\delta_{\xi}^{\prime}, \delta_{\tilde{\xi}}^{\prime}\right] D } & =U^{\mu} D_{\mu} D+2 i \xi \tilde{\xi}[\sigma, D]+2 \rho D+\frac{2 i}{3} \sigma\left(\xi \gamma^{\mu} \gamma^{\nu} \nabla_{\mu} \nabla_{\nu} \tilde{\xi}-\tilde{\xi} \gamma^{\mu} \gamma^{\nu} \nabla_{\mu} \nabla_{\nu} \xi\right)
\end{aligned}
$$

The last term vanishes given (A.40). Thus on gauge-covariant fields, the nonvanishing commutators are a sum of translation, gauge transformation, Lorentz rotation, dilatation, and $R$-rotation. ${ }^{30}$ For a fundamental chiral multiplet whose bottom component has scaling dimension $\Delta$, we have $\left[\delta_{\xi_{1}}^{\prime}, \delta_{\xi_{2}}^{\prime}\right]=\left[\delta_{\tilde{\xi}_{1}}^{\prime}, \delta_{\tilde{\xi}_{2}}^{\prime}\right]=0$ on all fields except for

$$
\left[\delta_{\tilde{\xi}_{1}}^{\prime}, \delta_{\tilde{\xi}_{2}}^{\prime}\right] F=\frac{4 \Delta}{3}\left(\tilde{\xi}_{1} \gamma^{\mu} \gamma^{\nu} \nabla_{\mu} \nabla_{\nu} \tilde{\xi}_{2}-\tilde{\xi}_{2} \gamma^{\mu} \gamma^{\nu} \nabla_{\mu} \nabla_{\nu} \tilde{\xi}_{1}\right) A,
$$

which vanishes provided that (A.40) is satisfied. We then have that

$$
\begin{aligned}
& {\left[\delta_{\xi}^{\prime}, \delta_{\tilde{\xi}}^{\prime}\right] A=U^{\mu} D_{\mu} A+2 i \xi \tilde{\xi} \sigma A+\Delta \rho A-\Delta \alpha A,} \\
& {\left[\delta_{\xi}^{\prime}, \delta_{\tilde{\xi}}^{\prime}\right] \psi=U^{\mu} D_{\mu} \psi+2 i \xi \tilde{\xi} \sigma \psi+\epsilon_{\mu \nu} \sigma^{\mu \nu} \psi+(\Delta+1 / 2) \rho \psi+(1-\Delta) \alpha \psi,} \\
& {\left[\delta_{\xi}^{\prime}, \delta_{\tilde{\xi}}^{\prime}\right] F=U^{\mu} D_{\mu} F+2 i \xi \tilde{\xi} \sigma F+(\Delta+1) \rho F+(2-\Delta) \alpha F .}
\end{aligned}
$$

Thus the $R$-charges are fixed by $\Delta$. Similar commutators hold for an antichiral multiplet. Finally, the curved-space Chern-Simons action is given by

$$
\left.\mathcal{L}_{\mathrm{CS}}\right|_{M^{3}}=\frac{k}{4 \pi i} \operatorname{Tr}\left[\sqrt{g}^{-1} \epsilon^{\mu \nu \rho}\left(A_{\mu} \partial_{\nu} A_{\rho}-\frac{2 i}{3} A_{\mu} A_{\nu} A_{\rho}\right)-2 \lambda \tilde{\lambda}+2 i D \sigma\right],
$$

\footnotetext{
${ }^{30}$ Under a Lorentz transformation $F_{\mu \nu} \rightarrow \Lambda_{\mu}{ }^{\alpha} \Lambda_{\nu}{ }^{\beta} F_{\alpha \beta}$ where $\Lambda_{\alpha \beta}=g_{\alpha \beta}+\epsilon_{\alpha \beta}+O\left(\epsilon^{2}\right)$ with $\epsilon_{\alpha \beta}=-\epsilon_{\beta \alpha}$, the change in $F_{\mu \nu}$ is $\epsilon_{\mu \alpha} F^{\alpha}{ }_{\nu}-\epsilon_{\nu \alpha} F^{\alpha}{ }_{\mu}$. The change in $\lambda$ under a rotation is $\epsilon^{\alpha \beta} \sigma_{\alpha \beta} \lambda$.
} 
integrated against the standard volume element $\sqrt{g} d^{3} x$. We compute that

$$
\left.\delta^{\prime} \mathcal{L}_{\mathrm{CS}}\right|_{M^{3}}=\frac{k}{4 \pi} \nabla_{\mu} \operatorname{Tr}\left[\sqrt{g}^{-1} \epsilon^{\mu \nu \rho}\left(\xi \gamma_{\nu} \tilde{\lambda}+\tilde{\xi} \gamma_{\nu} \lambda\right) A_{\rho}-2\left(\xi \gamma^{\mu} \tilde{\lambda}-\tilde{\xi} \gamma^{\mu} \lambda\right) \sigma\right]
$$

(compare to (5.14)). Aside from possible boundary conditions, no special conditions need to be imposed on $\xi$ and $\tilde{\xi}$ to ensure SUSY'-invariance of the Chern-Simons action.

\section{B Gauging SUSY NLSMs}

Here, we describe how to gauge a global symmetry (under which the fields do not transform in a linear representation) at the nonlinear level while preserving global SUSY.

\section{B.1 Kähler potential}

We first consider a supersymmetric sigma model described by a Kähler potential. We work in $0+1$ dimensions (the logic is the same in higher dimensions), namely $\mathcal{N}=2$ quantum mechanics with Kähler target space, and illustrate the logic with the simplest example of a $\mathbb{C P}^{1}$ sigma model parametrized by a single complex scalar, with isometry group $\mathrm{SU}(2)$ acting by linear fractional transformations (the example relevant for the main text). Let $\Phi$ be a $1 \mathrm{D} \mathcal{N}=2$ chiral superfield. In the patch containing the origin, $\mathbb{C P}^{1}$ has coordinates $\Phi, \Phi^{\dagger}$ with Kähler potential $K=\log \left(1+|\Phi|^{2}\right)$. To gauge the action $S=\int d t d^{2} \theta K$, consider infinitesimal local $\mathrm{SU}(2)$ transformations parametrized by chiral superfields $\Lambda_{i}$ : $\delta_{\mathrm{SU}(2)} \Phi=\Lambda_{i} X_{i}$. The corresponding change in $K$ is given by (4.15). We wish to cancel the terms involving $J_{i}$ in (4.15), leaving only a Kähler transformation, by means of an appropriate counterterm:

$$
K \rightarrow K+\Gamma\left(\Phi, \Phi^{\dagger}, V\right) .
$$

The construction of $\Gamma$ satisfying $\delta_{\mathrm{SU}(2)} \Gamma=i\left(\Lambda_{i}-\bar{\Lambda}_{i}\right) J_{i}$ is a special case of a more general problem. An action invariant under $H$ can be promoted to an action invariant under $G \supset H$ by adding a counterterm constructed out of the original fields and fields $V$ parametrizing the coset $G / H$, with the condition that the counterterm vanishes when $V=0$ :

$$
L_{G}(X, V)=L_{H}(X)+L_{\mathrm{ct}}(X, V), \quad L_{\mathrm{ct}}(X, 0)=0 .
$$

In particular, a vector superfield can be thought of as parametrizing $G_{\mathbb{C}} / G$, with gauge transformations corresponding to the action of $G_{\mathbb{C}}$ by left multiplication.

The interpretation of a vector superfield as a coset parameter proceeds as follows [41]. Complexified gauge parameters implement gauge transformations in $G_{\mathbb{C}}$. An arbitrary element of $G_{\mathbb{C}}$ can be written as

$$
g=e^{v_{i} T_{i}} e^{i u_{i} T_{i}}=(\text { Hermitian})(\text { unitary })
$$

where $u_{i}$ and $v_{i}$ are real and $T_{i}$ are Hermitian $\left(u_{i}\right.$ are coordinates on $G$, and $v_{i}$ are coordinates on $G_{\mathbb{C}} / G$ ). The left cosets of $G$ in $G_{\mathbb{C}} / G$ are thus represented by

$$
v=e^{v_{i} T_{i}}
$$


$G_{\mathbb{C}}$ acts naturally on $G_{\mathbb{C}} / G$ by left multiplication, which can be written as a combination of $G$ - and $G_{\mathbb{C}} / G$ - transformations $v^{\prime}=u_{0} v u_{0}^{-1}$ and $v^{\prime 2}=v_{0} v^{2} v_{0}$ parametrized by

$$
u_{0}=e^{i u_{0 i} T_{i}} \in G, \quad v_{0}=e^{v_{0 i} T_{i}} \in G_{\mathbb{C}} / G
$$

The infinitesimal form is

$$
\delta e^{2 v_{i} T_{i}}=i\left(u_{0 j}-i v_{0 j}\right) T_{j} e^{2 v_{i} T_{i}}-i e^{2 v_{i} T_{i}}\left(u_{0 j}+i v_{0 j}\right) T_{j} \equiv i \epsilon e^{2 v_{i} T_{i}}-i e^{2 v_{i} T_{i}} \bar{\epsilon}
$$

If we identify $\epsilon_{i}$ with the lowest component of $\Lambda_{i}$ and $v_{i}$ with the lowest component of $V_{i}$, then this transformation is simply the lowest component of the super gauge transformation

$$
\delta e^{2 V}=i \Lambda e^{2 V}-i e^{2 V} \bar{\Lambda} \Longleftrightarrow e^{2 V^{\prime}}=e^{i \Lambda} e^{2 V} e^{-i \bar{\Lambda}}
$$

where $V=V_{i} T_{i}$ and $\Lambda=\Lambda_{i} T_{i}$.

In our case of interest, $G=\mathrm{SU}(2)$ with $\mathrm{SU}(2)_{\mathbb{C}}=\mathrm{SL}(2, \mathbb{C})$. On a function of $\Phi, \Phi^{\dagger}, V$, a local $\mathrm{SU}(2)$ variation can be written as

$$
\delta_{\mathrm{SU}(2)}=\Lambda_{i} X_{i} \partial_{\Phi}+\bar{\Lambda}_{i} \bar{X}_{i} \partial_{\Phi^{\dagger}}+\delta_{\mathrm{SU}(2)} V \partial_{V}=\operatorname{Re}\left(\Lambda_{i}\right) \mathcal{P}_{i}+i \operatorname{Im}\left(\Lambda_{i}\right) \mathcal{O}_{i}
$$

where (suppressing variations of $V$ )

$$
\mathcal{P}_{i}=X_{i} \partial_{\Phi}+\bar{X}_{i} \partial_{\Phi^{\dagger}}+\cdots \equiv P_{i}+\cdots, \quad \mathcal{O}_{i}=X_{i} \partial_{\Phi}-\bar{X}_{i} \partial_{\Phi^{\dagger}}+\cdots \equiv O_{i}+\cdots
$$

(Re and Im are shorthand for the appropriate linear combinations of $\Lambda_{i}$ and $\bar{\Lambda}_{i}$, which are not chiral superfields). The $O_{i}$ satisfy $\left[O_{i}, O_{j}\right]=\epsilon_{i j k} O_{k}$ and $O_{i} J_{j}=O_{j} J_{i}=i\left(2 J_{i} J_{j}-\frac{1}{2} \delta_{i j}\right)$.

We focus on the $\mathcal{O}_{i}$ part in $\delta_{\mathrm{SU}(2)}$ because the undesirable terms in (4.15) involve $\operatorname{Im}\left(\Lambda_{i}\right)$. Clearly, $K$ is invariant (up to a Kähler transformation) under gauge transformations with $\Lambda_{i}$ purely real (i.e., under $G \subset G_{\mathbb{C}}$ ). With $\Lambda_{i}$ purely imaginary, gauge transformations (in the part of $G_{\mathbb{C}}$ not in $G$, namely $G_{\mathbb{C}} / G$ ) can be implemented by the $\mathcal{O}_{i}$. Now we do a formal manipulation (forgetting that the parameters must be chiral superfields): let $\Lambda_{i}=i R_{i}$ with $R_{i}$ real to isolate $\mathcal{O}_{i}$; then up to Kähler transformations,

$$
\delta_{\mathrm{SU}(2)} K=-i\left(\Lambda_{i}-\bar{\Lambda}_{i}\right) J_{i}=2 R_{i} J_{i}=i R_{i} \mathcal{O}_{i} K=i R_{i} O_{i} K
$$

To cancel this variation, we demand that $i R_{i} \mathcal{O}_{i} \Gamma=-2 R_{i} J_{i}$, subject to the boundary condition $\Gamma\left(\Phi, \Phi^{\dagger}, V=0\right)=0$. Exponentiating, we want:

$$
\left(e^{i R_{i} \mathcal{O}_{i}}-1\right) \Gamma=\frac{e^{i R_{i} \mathcal{O}_{i}}-1}{i R_{j} \mathcal{O}_{j}}\left(-2 R_{k} J_{k}\right)
$$

If we take $R_{i}=V_{i}$, then $e^{i R_{i} \mathcal{O}_{i}}$ transforms $V$ to zero. Thus by the boundary condition,

$$
\Gamma=\frac{e^{i V_{i} \mathcal{O}_{i}}-1}{i V_{j} \mathcal{O}_{j}}\left(2 V_{k} J_{k}\right)=\frac{e^{i V_{i} O_{i}}-1}{i V_{j} O_{j}}\left(2 V_{k} J_{k}\right)=2 \int_{0}^{1} d \alpha e^{i \alpha V_{i} O_{i}} V_{j} J_{j}
$$

where the derivatives in $\mathcal{O}$ do not act on the $V$, thus justifying the replacement $\mathcal{O} \rightarrow O$. For completeness, one should check that $\mathcal{P}_{i} \Gamma=0$, which we do not do explicitly here. 


\section{B.2 Higher-derivative terms}

Our applications require gauging supersymmetric sigma models containing higherderivative terms involving the Grassmann-odd superfield $D \Phi$. Concretely, consider $K^{\prime}$ in (4.21), associated to which are fermionic Noether currents as in (4.23), for which we wish to construct a counterterm $\Gamma^{\prime}$ satisfying (4.24). First define the bosonic operators

$$
O_{i}^{\prime}=X_{i} \partial_{\Phi}-\bar{X}_{i} \partial_{\Phi^{\dagger}}+D X_{i} \partial_{D \Phi}-\left(D X_{i}\right)^{\dagger} \partial_{(D \Phi)^{\dagger}} \Longrightarrow O_{i}^{\prime} K^{\prime}=-2 i J_{i}^{\prime}
$$

as well as the fermionic operators

$$
P_{i}=X_{i} \partial_{D \Phi} \Longrightarrow P_{i} K^{\prime}=-i I_{i}, P_{i}^{\dagger} K^{\prime}=i I_{i}^{\dagger}
$$

(note that $D^{\dagger} \Phi^{\dagger}=-(D \Phi)^{\dagger}$ and $\left.\left(\partial_{D \Phi}\right)^{\dagger}=-\partial_{(D \Phi)^{\dagger}}\right)$. We may write

$$
\delta_{\mathrm{SU}(2)} K^{\prime}=-i\left(\Lambda_{i}-\Lambda_{i}^{\dagger}\right) J_{i}^{\prime}-i D\left(\Lambda_{i}-\Lambda_{i}^{\dagger}\right) I_{i}-i D^{\dagger}\left(\Lambda_{i}-\Lambda_{i}^{\dagger}\right) I_{i}^{\dagger}
$$

by virtue of $D \Lambda_{i}^{\dagger}=D^{\dagger} \Lambda_{i}=0$, so that if $\Lambda_{i}=i \xi_{i}$ with $\xi_{i}$ real, then

$$
\begin{aligned}
\delta_{\mathrm{SU}(2)} K^{\prime} & =2\left(\xi_{i} J_{i}^{\prime}+D \xi_{i} I_{i}+D^{\dagger} \xi_{i} I_{i}^{\dagger}\right) \\
& =i\left(\xi_{i} O_{i}^{\prime}+2 D \xi_{i} P_{i}-2 D^{\dagger} \xi_{i} P_{i}^{\dagger}\right) K^{\prime} .
\end{aligned}
$$

To cancel this variation, we demand that

$$
i\left(\xi_{i} O_{i}^{\prime}+2 D \xi_{i} P_{i}-2 D^{\dagger} \xi_{i} P_{i}^{\dagger}\right) \Gamma^{\prime}=-2\left(\xi_{i} J_{i}^{\prime}+D \xi_{i} I_{i}+D^{\dagger} \xi_{i} I_{i}^{\dagger}\right),
$$

subject to the usual boundary condition. This exponentiates to give

$$
\left(e^{i\left(\xi_{i} O_{i}^{\prime}+2 D \xi_{i} P_{i}-2 D^{\dagger} \xi_{i} P_{i}^{\dagger}\right)}-1\right) \Gamma^{\prime}=-\frac{e^{i\left(\xi_{i} O_{i}^{\prime}+2 D \xi_{i} P_{i}-2 D^{\dagger} \xi_{i} P_{i}^{\dagger}\right)}-1}{i\left(\xi_{j} O_{j}^{\prime}+2 D \xi_{j} P_{j}-2 D^{\dagger} \xi_{j} P_{j}^{\dagger}\right)} 2\left(\xi_{k} J_{k}^{\prime}+D \xi_{k} I_{k}+D^{\dagger} \xi_{k} I_{k}^{\dagger}\right) .
$$

Setting $\xi_{i}=V_{i}$, the boundary condition yields

$$
\begin{aligned}
\Gamma^{\prime} & =\frac{e^{i\left(V_{i} O_{i}^{\prime}+2 D V_{i} P_{i}-2 D^{\dagger} V_{i} P_{i}^{\dagger}\right)}-1}{i\left(V_{j} O_{j}^{\prime}+2 D V_{j} P_{j}-2 D^{\dagger} V_{j} P_{j}^{\dagger}\right)} 2\left(V_{k} J_{k}^{\prime}+D V_{k} I_{k}+D^{\dagger} V_{k} I_{k}^{\dagger}\right) \\
& =2 \int_{0}^{1} d \alpha e^{i \alpha\left(V_{i} O_{i}^{\prime}+2 D V_{i} P_{i}-2 D^{\dagger} V_{i} P_{i}^{\dagger}\right)}\left(V_{j} J_{j}^{\prime}+D V_{j} I_{j}+D^{\dagger} V_{j} I_{j}^{\dagger}\right) .
\end{aligned}
$$

\section{Details on the $\mathcal{N}=2$ coadjoint orbit}

\section{C.1 $\mathbb{C P}^{1}$ sigma model}

Our conventions for the geometry of $\mathbb{C P}^{1}$ are as follows. Stereographic projection yields the relation $z=e^{i \varphi} / \tan (\theta / 2)$ between the coordinate $z$ on $\mathbb{C P}^{1}$ and spherical coordinates $(\theta, \varphi)$ on $S^{2}$ (we use $\varphi$ to avoid confusion with $\phi$ ). We thus get

$$
d s^{2}=\frac{\operatorname{Re}(d z \otimes d \bar{z})}{\left(1+|z|^{2}\right)^{2}}=\frac{1}{4}\left(d \theta^{2}+\sin ^{2} \theta d \varphi^{2}\right),
$$


the round metric on $S^{2}$ of radius 1/2. In this setup, the projection is done from the north pole of the $S^{2}$ at $(0,0,1)^{T}$. The adjoint action of $\mathrm{SU}(2)$ on $\mathfrak{s u}(2) \cong \mathbb{R}^{3}$ descends to an action on $S^{2} \subset \mathbb{R}^{3}$, giving rise to a two-to-one map $\mathrm{SU}(2) \rightarrow \mathrm{SO}(3)$ :

$$
\pm\left(\begin{array}{cc}
a & b \\
-\bar{b} & \bar{a}
\end{array}\right) \mapsto\left(\begin{array}{ccc}
\operatorname{Re}\left(a^{2}-b^{2}\right) & \operatorname{Im}\left(a^{2}+b^{2}\right) & -2 \operatorname{Re}(a b) \\
-\operatorname{Im}\left(a^{2}-b^{2}\right) & \operatorname{Re}\left(a^{2}+b^{2}\right) & 2 \operatorname{Im}(a b) \\
2 \operatorname{Re}(a \bar{b}) & 2 \operatorname{Im}(a \bar{b}) & |a|^{2}-|b|^{2}
\end{array}\right) .
$$

Alternatively, $\mathrm{SU}(2)$ acts on $\mathbb{C P}^{1}$ by linear fractional transformations:

$$
z \rightarrow \frac{a z+b}{-\bar{b} z+\bar{a}}, \quad K \rightarrow K-\log (\bar{a}-\bar{b} z)-\log (a-b \bar{z}) .
$$

Combined with stereographic projection, this results in the following map $\mathrm{SU}(2) \rightarrow \mathrm{SO}(3)$ :

$$
\pm\left(\begin{array}{cc}
a & b \\
-\bar{b} & \bar{a}
\end{array}\right) \mapsto\left(\begin{array}{ccc}
\operatorname{Re}\left(a^{2}-b^{2}\right) & -\operatorname{Im}\left(a^{2}+b^{2}\right) & -2 \operatorname{Re}(a b) \\
\operatorname{Im}\left(a^{2}-b^{2}\right) & \operatorname{Re}\left(a^{2}+b^{2}\right) & -2 \operatorname{Im}(a b) \\
2 \operatorname{Re}(a \bar{b}) & -2 \operatorname{Im}(a \bar{b}) & |a|^{2}-|b|^{2}
\end{array}\right) .
$$

We use the latter convention. The sign differences between (C.2) and (C.4) have the following consequence. The point $(\sin \theta \cos \varphi, \sin \theta \sin \varphi, \cos \theta)^{T}$ of $S^{2}$ transforms via the image of (4.10) under the map (C.4), which coincides with the action of the standard rotation generators

$$
\vec{D}=-i\left(-\sin \varphi \partial_{\theta}-\cos \varphi \cot \theta \partial_{\varphi}, \cos \varphi \partial_{\theta}-\sin \varphi \cot \theta \partial_{\varphi}, \partial_{\varphi}\right),
$$

satisfying $\left[D_{i}, D_{j}\right]=i \epsilon_{i j k} D_{k}$, only after flipping the sign of $\epsilon_{2}$ in (4.10). Finally, by "Hopf map," we mean the map $\mathrm{SU}(2) \rightarrow S^{2}$ that sends a given element of $\mathrm{SU}(2)$ to the point to which it sends the north pole $(0,0,1)^{T}$ of $S^{2}$, according to (C.4). Stereographic projection then allows us to identify an $\mathrm{SU}(2)$ element with the point $z=-a / \bar{b}$ of $\mathbb{C P}^{1}$.

In writing (3.9) as a sigma model to $\mathbb{C P}^{1}$, we identified $g=\left(\begin{array}{cc}a & b \\ -\bar{b} & \bar{a}\end{array}\right)=\left(\begin{array}{cc}a & \frac{r}{a} \\ -r & \bar{a}\end{array}\right)$ with the complex scalar $\phi$ (playing the role of $z$ in the previous paragraph) via the Hopf map and an appropriate (partial) gauge fixing. To see that the map (4.5) is equivariant with respect to the action of $\mathrm{SU}(2)$, consider an arbitrary $\mathrm{SU}(2)$ transformation, either global or local:

$$
g \rightarrow\left(\begin{array}{cc}
a_{\ell} & b_{\ell} \\
-\bar{b}_{\ell} & \bar{a}_{\ell}
\end{array}\right) g=\left(\begin{array}{cc}
a_{\ell} a-b_{\ell} r & a_{\ell} r+b_{\ell} \bar{a} \\
-\bar{a}_{\ell} r-\bar{b}_{\ell} a & \bar{a}_{\ell} \bar{a}-\bar{b}_{\ell} r
\end{array}\right)
$$

(" $\ell$ " stands for "left"). To preserve the reality condition on $b$, as required by (4.5), we must again gauge away the phase of the off-diagonal components. This is achieved by multiplying $a_{\ell} a-b_{\ell} r$ by $-e^{i \theta}$ and $a_{\ell} r+b_{\ell} \bar{a}$ by $-e^{-i \theta}$ where $\theta$ is the phase of $a_{\ell} r+b_{\ell} \bar{a}$ (the minus sign preserves the " $(a, r)=(+,-)$ " convention), so that

$$
a \rightarrow a^{\prime}=-\frac{\left(a_{\ell} a-b_{\ell} r\right)\left(a_{\ell} r+b_{\ell} \bar{a}\right)}{\left|a_{\ell} r+b_{\ell} \bar{a}\right|}, \quad r \rightarrow r^{\prime}=-\left|a_{\ell} r+b_{\ell} \bar{a}\right|, \quad \phi \rightarrow \phi^{\prime}=\frac{a_{\ell} \phi+b_{\ell}}{-\bar{b}_{\ell} \phi+\bar{a}_{\ell}}
$$

under SU(2). Using (4.5), we indeed find that $\left(a^{\prime}, r^{\prime}\right)=\left(\phi^{\prime},-1\right) / \sqrt{1+\left|\phi^{\prime}\right|^{2}}$, for which the minus sign in $r(\phi)$ is crucial. 
Now, as in (4.10), let us drop the $\ell$ subscripts on the $\mathrm{SU}(2)$ transformation parameters. Under global SU(2), the Wess-Zumino term $\mathcal{L}_{0}$ in (4.7) picks up a total derivative,

$$
\mathcal{L}_{0} \rightarrow \mathcal{L}_{0}+i \partial_{t} \log \left(\frac{-\bar{b} \phi+\bar{a}}{-b \phi^{\dagger}+a}\right)
$$

while $\mathcal{L}_{A}$ in (4.8) is invariant. Under local $\mathrm{SU}(2)$, the variation of their sum $\mathcal{L}=\mathcal{L}_{0}+\mathcal{L}_{A}$ (more conveniently written in terms of the $\epsilon_{i}$ in (4.10)) is

$$
\delta_{\mathrm{SU}(2)} \mathcal{L}=\partial_{t}\left(\epsilon_{3}-\frac{\epsilon_{1}+i \epsilon_{2}}{2} \phi-\frac{\epsilon_{1}-i \epsilon_{2}}{2} \phi^{\dagger}\right) .
$$

Thus the SU(2) gauge invariance of the $g$ Lagrangian (3.9) only holds up to total derivatives in the $\phi$ Lagrangian $\mathcal{L}$ due to the necessity of gauge-fixing the action of $\mathrm{U}(1)$ on the right. Upon promoting $\phi$ to the superfield $\Phi$, global SU(2) acts as

$$
\Phi \rightarrow \frac{a \Phi+b}{-\bar{b} \Phi+\bar{a}} \Longleftrightarrow(\phi, \psi, \dot{\phi}) \rightarrow\left(\frac{a \phi+b}{-\bar{b} \phi+\bar{a}}, \frac{\psi}{(-\bar{b} \phi+\bar{a})^{2}}, \frac{\dot{\phi}}{(-\bar{b} \phi+\bar{a})^{2}}\right),
$$

under which the $\psi^{\dagger} \psi$ term in $\tilde{\mathcal{L}}_{0}(4.9)$ and the $\dot{\phi} \dot{\phi}^{\dagger}$ term in $\mathcal{L}^{\prime}(4.21)$ are invariant.

\section{C.2 Effective action}

Upon changing variables to $\psi^{\prime}=\psi /\left(1+|\phi|^{2}\right)$, the path integral for $\tilde{\mathcal{L}}_{\text {tot }}$ acquires a Jacobian that renormalizes the action by $-2 \operatorname{tr} \log \left(1+|\phi|^{2}\right)$. Integrating by parts, we have

$$
\tilde{\mathcal{L}}_{\text {tot }} \supset j \mathcal{L}+\frac{i\left(\psi^{\dagger} \dot{\psi}-\dot{\psi}^{\dagger} \psi\right)}{2 \mu\left(1+|\phi|^{2}\right)^{2}}+\frac{(\mathcal{L} / \mu-j) \psi^{\dagger} \psi}{\left(1+|\phi|^{2}\right)^{2}}=j \mathcal{L}+\psi^{\prime \dagger}\left(\frac{i \partial_{t}+\mathcal{L}}{\mu}-j\right) \psi^{\prime} .
$$

Performing the path integral over $\psi$ thus generates the effective action

$$
\operatorname{tr} \log \left(\frac{i \partial_{t}}{\mu}-j\right)+\sum_{n=1}^{\infty} \frac{(-1)^{n+1}}{n} \operatorname{tr} X^{n}
$$

where $X \equiv\left(i \partial_{t}-\mu^{\prime}\right)^{-1} \mathcal{L}$ and $\mu^{\prime} \equiv j \mu$. Inserting complete sets of states ${ }^{31}$ yields

$$
\begin{aligned}
\operatorname{tr} X^{n} & =\int \frac{d t_{1} \cdots d t_{n} d E_{1} \cdots d E_{n}}{(2 \pi)^{2 n-1}}\left\langle t_{n} \mid E_{1}\right\rangle\left\langle E_{1}\left|\left(\frac{\mathcal{L}\left(t_{1}\right)}{E_{1}-\mu^{\prime}}\right)\right| t_{1}\right\rangle\left\langle t_{1}|\cdots| E_{n}\right\rangle\left\langle E_{n}\left|\left(\frac{\mathcal{L}\left(t_{n}\right)}{E_{n}-\mu^{\prime}}\right)\right| t_{n}\right\rangle \\
& =\int \frac{d E_{1} \cdots d E_{n-1}}{(2 \pi)^{n-1}} G\left(E_{1}, \ldots, E_{n-1}\right) \tilde{\mathcal{L}}\left(E_{1}\right) \cdots \tilde{\mathcal{L}}\left(E_{n-1}\right) \tilde{\mathcal{L}}\left(-E_{1}-\cdots-E_{n-1}\right)
\end{aligned}
$$

with $\tilde{\mathcal{L}}(E) \equiv \frac{1}{2 \pi} \int d t e^{i E t} \mathcal{L}(t)$ and integral kernel

$$
G\left(E_{1}, \ldots, E_{n-1}\right)=\int \frac{d E}{E\left(E+E_{1}\right) \cdots\left(E+E_{1}+\cdots+E_{n-1}\right)},
$$

whose $\mu^{\prime}$-dependence drops out after suitably redefining $E$. For $n>1$, one can regulate this integral by shifting the contour of integration to $\mathbb{R} \pm i \epsilon$, in which case it obviously

\footnotetext{
${ }^{31}$ Normalized as $\langle t \mid E\rangle=e^{-i E t} \Longrightarrow \int \frac{d E}{2 \pi}|E\rangle\left\langle E\left|=\int \frac{d t}{2 \pi}\right| t\right\rangle\langle t|=1$.
} 
vanishes (the integrand falls off faster than $1 / E$, and the sum of the residues vanishes). When $n=1$,

$$
\operatorname{tr} X=\int \frac{d t d E}{2 \pi}\langle t \mid E\rangle\left\langle E\left|\left(\frac{\mathcal{L}}{i \partial_{t}-\mu^{\prime}}\right)\right| t\right\rangle=\frac{1}{2 \pi} \int \frac{d E}{E} \int d t \mathcal{L} .
$$

In this case, the $E$ integral evaluates to $\pm i \pi$ depending on how we shift the pole away from the real line. Hence the only relevant term in the one-loop effective action is

$$
\operatorname{tr} X= \pm \frac{i}{2} \int d t \mathcal{L}
$$

namely the tadpole with one external $\mathcal{L}$ leg (we have ignored the vacuum energy).

\section{C.3 Canonical quantization}

\section{C.3.1 Bosonic system}

We begin by working in spherical coordinates $(\theta, \varphi)$ to make the connection to the monopole problem manifest. It is instructive to quantize the system $L_{B}$ in arbitrary gauge. For simplicity, we consider only longitudinal gauges for the monopole vector potential, parametrized by $\alpha$ in (4.31), that preserve the classical U(1) symmetry manifest when $\alpha=0$ :

$$
\vec{A}=\frac{(j-\alpha)+j \cos \theta}{r \sin \theta} \hat{\varphi}
$$

The gauges $S, E$, and $N$ that we have defined by setting $\alpha=(0, j, 2 j)$ are good near the south pole $(=0)$, equator, and north pole $(=\infty)$, respectively.

At finite $\mu$, the phase space is $(2+2)$-dimensional. The classical Lagrangian (4.31) is

$$
L_{B}=((j-\alpha)+j \cos \theta) \dot{\varphi}+\frac{\dot{\theta}^{2}}{2 \mu}+\frac{\sin ^{2} \theta \dot{\varphi}^{2}}{2 \mu},
$$

from which we obtain the canonical momenta and classical Hamiltonian

$$
\pi_{\theta}=\frac{\dot{\theta}}{\mu}, \quad \pi_{\varphi}=(j-\alpha)+j \cos \theta+\frac{\sin ^{2} \theta \dot{\varphi}}{\mu}, \quad H_{\mathrm{cl}}=\frac{\mu}{2}\left[\left(\frac{\pi_{\varphi}-(j-\alpha)-j \cos \theta}{\sin \theta}\right)^{2}+\pi_{\theta}^{2}\right] .
$$

The "good" angular momentum operators $\vec{L}$ in the presence of a monopole are well-known generalizations of the standard $\vec{D}$ in (C.5), giving the quantum Hamiltonian (4.33) (with a subscript $j$ to emphasize the spin) where

$$
\vec{L}=\vec{D}+\left(\frac{(j+(j-\alpha) \cos \theta) \cos \varphi}{\sin \theta}, \frac{(j+(j-\alpha) \cos \theta) \sin \varphi}{\sin \theta}, \alpha-j\right)
$$

which satisfy $\left[L_{i}, L_{j}\right]=i \epsilon_{i j k} L_{k}$. The corresponding classical expressions $\vec{L}_{\mathrm{cl}}$, obtained simply by substituting $\pi_{\theta}$ and $\pi_{\varphi}$ for $-i \partial_{\theta}$ and $-i \partial_{\varphi}$ in (C.5) and (C.15), satisfy the expected Poisson brackets $\left[\left(L_{i}\right)_{\mathrm{cl}},\left(L_{j}\right)_{\mathrm{cl}}\right]_{\mathrm{PB}}=\epsilon_{i j k}\left(L_{k}\right)_{\mathrm{cl}}$ with respect to $\left(\theta, \pi_{\theta}, \varphi, \pi_{\varphi}\right) \cdot{ }^{32}$

\footnotetext{
${ }^{32}$ The $\vec{L}_{\mathrm{cl}}$ can also be written in a gauge-independent manner in terms of the velocities $\dot{\theta}, \dot{\varphi}$ :

$$
\vec{L}_{\mathrm{cl}}=j(\sin \theta \cos \varphi, \sin \theta \sin \varphi, \cos \theta)+O(1 / \mu) .
$$
}

In this form, the $\mu \rightarrow \infty$ limit is manifest. 
At $\mu=\infty$, the phase space is $(1+1)$-dimensional and we have $\pi_{\varphi}=(j-\alpha)+j \cos \theta$, $\pi_{\theta}=0$. Hence there is a distinguished polarization in which $\varphi$ is the canonical coordinate:

$$
\left(L_{1}\right)_{\mathrm{cl}}=\cos \varphi \sqrt{j^{2}-\left(L_{3}\right)_{\mathrm{cl}}^{2}}, \quad\left(L_{2}\right)_{\mathrm{cl}}=\sin \varphi \sqrt{j^{2}-\left(L_{3}\right)_{\mathrm{cl}}^{2}}, \quad\left(L_{3}\right)_{\mathrm{cl}}=\pi_{\varphi}-(j-\alpha) .
$$

The Poisson brackets on the reduced phase space $\left(\varphi, \pi_{\varphi}\right)$ take the same form as for finite $\mu$. The corresponding quantum operators satisfying $\left[L_{3}, L_{ \pm}\right]= \pm L_{ \pm}$and $\left[L_{+}, L_{-}\right]=2 L_{3}$ are

$$
L_{ \pm}=\sqrt{j \pm L_{3}} e^{ \pm i \varphi} \sqrt{j \mp L_{3}}, \quad L_{3}=-i \partial_{\varphi}-(j-\alpha),
$$

where we have set $L_{ \pm}=L_{1} \pm i L_{2}$.

It is easy to recast the above statements in complex coordinates $\left(\phi, \phi^{\dagger}\right)$. At finite $\mu$, we immediately obtain the quantum angular momentum operators (4.34). Their classical counterparts $\left(L_{i}\right)_{\mathrm{cl}}$, obtained simply by substituting $\pi_{\phi}$ for $-i \partial_{\phi}$ and $\pi_{\phi^{\dagger}}$ for $-i \partial_{\phi^{\dagger}}$, satisfy the expected Poisson brackets on the full phase space $\left(\phi, \pi_{\phi}, \phi^{\dagger}, \pi_{\phi^{\dagger}}\right)$. On the other hand, the classical canonical momenta following from (4.31) are

$$
\pi_{\phi}=\left(\pi_{\phi^{\dagger}}\right)^{\dagger}=-\frac{i j \phi^{\dagger}}{1+|\phi|^{2}}+\frac{i \alpha}{2 \phi}+\frac{2 \dot{\phi}^{\dagger}}{\mu\left(1+|\phi|^{2}\right)^{2}} .
$$

While canonical quantization does not fix the quantum representations of these momenta, we infer with the aid of (C.18) that the quantization rules for the velocities are

$$
\begin{gathered}
\frac{2 i \dot{\phi}^{\dagger}}{\mu} \stackrel{q}{\rightarrow}\left(1+|\phi|^{2}\right)^{2} \frac{\partial}{\partial \phi}-j \phi^{\dagger}\left(1+|\phi|^{2}\right)+\frac{\alpha}{2 \phi}\left(1+|\phi|^{2}\right)^{2}, \\
-\frac{2 i \dot{\phi}}{\mu} \stackrel{q}{\rightarrow}-\left(1+|\phi|^{2}\right)^{2} \frac{\partial}{\partial \phi^{\dagger}}-j \phi\left(1+|\phi|^{2}\right)+\frac{\alpha}{2 \phi^{\dagger}}\left(1+|\phi|^{2}\right)^{2},
\end{gathered}
$$

where " $\stackrel{q}{\rightarrow}$ " means "is represented quantumly by." After accounting for ordering ambiguities in the classical Hamiltonian, these rules indeed lead to the correct quantum Hamiltonian (4.33). At $\mu=\infty, \phi$ and $\phi^{\dagger}$ are no longer independent canonical variables. Letting the coordinate be $\phi$, we distinguish the corresponding momentum on the reduced phase space from that in (C.18) with an extra subscript: $\left(\pi_{\phi}\right)_{\text {red }}=2 \lim _{\mu \rightarrow \infty} \pi_{\phi}$ (the factor of two comes from integration by parts), in terms of which

$$
\left(L_{+}\right)_{\mathrm{cl}}=-i \phi^{2}\left(\pi_{\phi}\right)_{\mathrm{red}}+(2 j-\alpha) \phi, \quad\left(L_{-}\right)_{\mathrm{cl}}=i\left(\pi_{\phi}\right)_{\mathrm{red}}+\frac{\alpha}{\phi}, \quad\left(L_{3}\right)_{\mathrm{cl}}=i \phi\left(\pi_{\phi}\right)_{\mathrm{red}}-(j-\alpha) .
$$

Note that this holomorphic polarization differs from the $\varphi$-based polarization that we used in spherical coordinates, which treats $\phi$ and $\phi^{\dagger}$ equally. The Poisson brackets with respect to $\left(\phi,\left(\pi_{\phi}\right)_{\text {red }}\right)$ are then as expected: $\left[\left(L_{3}\right)_{\mathrm{cl}},\left(L_{ \pm}\right)_{\mathrm{cl}}\right]_{\mathrm{PB}}=\mp i\left(L_{ \pm}\right)_{\mathrm{cl}}$ and $\left[\left(L_{+}\right)_{\mathrm{cl}},\left(L_{-}\right)_{\mathrm{cl}}\right]_{\mathrm{PB}}=$ $-2 i\left(L_{3}\right)_{\mathrm{cl}}$. Representing $\left(\pi_{\phi}\right)_{\text {red }}$ by $-i \partial_{\phi}$ gives the correct quantum operators (4.35).

\section{C.3.2 Supersymmetric system}

Here, for simplicity, we set $\alpha=0$ ( $S$ gauge), keep $\mu$ finite, and work in complex coordinates $\phi, \phi^{\dagger}$. The canonical momenta for $L_{B}+L_{F}$ are

$$
\pi_{\phi}=-\left[j+\frac{\psi^{\dagger} \psi}{\mu\left(1+|\phi|^{2}\right)^{2}}\right] \frac{i \phi^{\dagger}}{1+|\phi|^{2}}+\frac{2 \dot{\phi}^{\dagger}}{\mu\left(1+|\phi|^{2}\right)^{2}}, \quad \pi_{\psi}=\frac{i \psi^{\dagger}}{2 \mu\left(1+|\phi|^{2}\right)^{2}},
$$


where $\pi_{\phi^{\dagger}}=\pi_{\phi}^{\dagger}$ and $\pi_{\psi^{\dagger}}=-\pi_{\psi}^{\dagger}$ (note that $\psi$ and $\psi^{\dagger}$ are not independent canonical coordinates). Defining $\chi$ as in (4.36), the classical Hamiltonian can be written as

$$
H_{\mathrm{cl}}^{\prime}=\frac{\mu}{2}\left(1+|\phi|^{2}\right)^{2}\left[\pi_{\phi}+\left(j+\chi^{\dagger} \chi\right) \frac{i \phi^{\dagger}}{1+|\phi|^{2}}\right]\left[\pi_{\phi^{\dagger}}-\left(j+\chi^{\dagger} \chi\right) \frac{i \phi}{1+|\phi|^{2}}\right]+j \mu \chi^{\dagger} \chi
$$

To quantize, we impose the canonical (anti)commutation relations

$$
\left[\phi, \pi_{\phi}\right]=\left[\phi^{\dagger}, \pi_{\phi^{\dagger}}\right]=i, \quad\left\{\chi, \chi^{\dagger}\right\}=1 .
$$

The last relation introduces (in addition to the ambiguities already present when quantizing $H_{\mathrm{cl}}$ ) fermion ordering ambiguities in $H_{\mathrm{cl}}^{\prime}$, which allow us to determine the quantum Hamiltonian corresponding to $H_{\mathrm{cl}}^{\prime}$ up to two constants $c_{1,2}$ :

$$
H^{\prime}=H_{j-c_{1}}+\frac{\mu}{2}\left(1+|\phi|^{2}\right)\left[2 j+\frac{\left(1-2 c_{1}\right)|\phi|^{2}}{1+|\phi|^{2}}-\left(\phi \frac{\partial}{\partial \phi}-\phi^{\dagger} \frac{\partial}{\partial \phi^{\dagger}}\right)\right] \chi^{\dagger} \chi-c_{2} j \mu
$$

Hermiticity requires that $c_{1,2}$ be real numbers, and the Dirac quantization condition further requires that $c_{1}$ be a half-integer. It turns out that $H^{\prime}$ can be diagonalized separately in the bosonic and fermionic sectors of the Hilbert space

$$
\left(L^{2}\left(S^{2}, \mathbb{C}\right) \otimes|0\rangle\right) \oplus\left(L^{2}\left(S^{2}, \mathbb{C}\right) \otimes \chi^{\dagger}|0\rangle\right)
$$

by the "good" angular momentum operators (4.34):

$$
\begin{aligned}
H^{\prime} & =\left(\begin{array}{cc}
H_{j-c_{1}}-c_{2} j \mu & 0 \\
0 & H_{j-c_{1}+1}+\left(1-c_{2}\right) j \mu
\end{array}\right) \\
& =\frac{\mu}{2}\left(\begin{array}{cc}
\ell_{b}\left(\ell_{b}+1\right)-\left(j-c_{1}\right)^{2}-2 c_{2} j & 0 \\
0 & \ell_{f}\left(\ell_{f}+1\right)-\left(j-c_{1}+1\right)^{2}+2\left(1-c_{2}\right) j
\end{array}\right),
\end{aligned}
$$

where $\ell_{b} \geq j-c_{1}$ and $\ell_{f} \geq j-c_{1}+1$. The constants $c_{1,2}$ are then uniquely fixed by demanding that the quantum theory be supersymmetric, namely that each positive energy level have equal numbers of bosonic and fermionic states and that SUSY not be spontaneously broken. Under these conditions, we find that $c_{1}=c_{2}+1 / 4 j=1 / 2$, leading to precisely the quantum Hamiltonian stated in (4.40). In particular, $\ell_{b, f}$ are half-integers and the $2 j$ bosonic ground states of zero energy occur at $\ell_{b}=j-1 / 2$. Having fixed $c_{1,2}$, it is convenient to note that the "fermionic" monopole angular momenta

$$
\vec{L}_{f}=\left.\vec{L}\right|_{j+\chi^{\dagger} \chi-1 / 2} \equiv \vec{D}+\left(j+\chi^{\dagger} \chi-1 / 2\right)\left(\frac{\phi+\phi^{\dagger}}{2}, \frac{\phi-\phi^{\dagger}}{2 i},-1\right)
$$

(compare to (C.15)), where

$$
\vec{D}=\left(\frac{1-\phi^{2}}{2} \frac{\partial}{\partial \phi}-\frac{1-\left(\phi^{\dagger}\right)^{2}}{2} \frac{\partial}{\partial \phi^{\dagger}},-\frac{1+\phi^{2}}{2 i} \frac{\partial}{\partial \phi}-\frac{1+\left(\phi^{\dagger}\right)^{2}}{2 i} \frac{\partial}{\partial \phi^{\dagger}}, \phi \frac{\partial}{\partial \phi}-\phi^{\dagger} \frac{\partial}{\partial \phi^{\dagger}}\right)
$$

in complex coordinates, diagonalize $H^{\prime}$ as in (4.38). 
Classically, the supercharges follow from the Noether procedure: ${ }^{33}$

$$
Q=\frac{2 i \dot{\phi}^{\dagger} \psi}{\mu\left(1+|\phi|^{2}\right)^{2}}, \quad Q^{\dagger}=-\frac{2 i \dot{\phi} \psi^{\dagger}}{\mu\left(1+|\phi|^{2}\right)^{2}} .
$$

Quantumly, the supercharges are represented by the nilpotent operators (4.37), which are adjoints with respect to the Fubini-Study measure on the sphere:

$$
\sin \theta d \varphi \wedge d \theta=\frac{2 i d \phi \wedge d \phi^{\dagger}}{\left(1+|\phi|^{2}\right)^{2}}
$$

In deriving (4.37), ordering ambiguities are fixed by demanding that $\frac{1}{2}\left\{Q, Q^{\dagger}\right\}=H^{\prime}$, where $H^{\prime}$ is known from (4.40). One can verify that both $Q, Q^{\dagger}$ annihilate the ground states of $H^{\prime}$ and commute with the $\left(L_{f}\right)_{i}$ in (C.29). Analysis of the supersymmetry transformations then shows that the velocities are represented by ${ }^{34}$

$$
\begin{gathered}
\frac{2 i \dot{\phi}^{\dagger}}{\mu} \stackrel{q}{\rightarrow}\left(1+|\phi|^{2}\right)^{2} \frac{\partial}{\partial \phi}-\left(j+\chi^{\dagger} \chi-1 / 2\right) \phi^{\dagger}\left(1+|\phi|^{2}\right), \\
-\frac{2 i \dot{\phi}}{\mu} \stackrel{q}{\rightarrow}-\left(1+|\phi|^{2}\right)^{2} \frac{\partial}{\partial \phi^{\dagger}}-\left(j+\chi^{\dagger} \chi-1 / 2\right) \phi\left(1+|\phi|^{2}\right) .
\end{gathered}
$$

These relations are consistent with the representations (4.37) of the supercharges as quantum operators if we choose the following ordering:

$$
Q=\frac{\sqrt{\mu} \chi}{1+|\phi|^{2}}\left(\frac{2 i \dot{\phi}^{\dagger}}{\mu}\right), \quad Q^{\dagger}=\left(-\frac{2 i \dot{\phi}}{\mu}\right) \frac{\sqrt{\mu} \chi^{\dagger}}{1+|\phi|^{2}},
$$

where the parenthesized expressions are understood as differential operators acting on the left. This understanding allows us to identify the ordering prescription needed to directly quantize the classical Hamiltonian, written in terms of velocities as

$$
H_{\mathrm{cl}}^{\prime}=H_{\mathrm{cl}}+j \mu \chi^{\dagger} \chi, \quad H_{\mathrm{cl}}=\frac{2 \dot{\phi} \dot{\phi}^{\dagger}}{\mu\left(1+|\phi|^{2}\right)^{2}} .
$$

We have seen that the quantum Hamiltonian is

$$
H^{\prime}=\frac{1}{2}\left\{Q, Q^{\dagger}\right\}=H_{j+\chi^{\dagger} \chi-1 / 2}+j \mu \chi^{\dagger} \chi-\frac{\mu}{2}(j-1 / 2),
$$

where

$$
H_{j}=-\frac{\mu}{2}\left[\left(1+|\phi|^{2}\right)^{2} \frac{\partial}{\partial \phi} \frac{\partial}{\partial \phi^{\dagger}}+j\left(1+|\phi|^{2}\right)\left(\phi \frac{\partial}{\partial \phi}-\phi^{\dagger} \frac{\partial}{\partial \phi^{\dagger}}\right)-j^{2}|\phi|^{2}\right]
$$

in complex coordinates. Applying (C.33) and (C.34) to the symmetrized expression

$$
H_{\mathrm{cl}}=\frac{\mu}{4}\left[\left(\frac{2 i \dot{\phi}^{\dagger}}{\mu}\right) \frac{1}{\left(1+|\phi|^{2}\right)^{2}}\left(-\frac{2 i \dot{\phi}}{\mu}\right)+\left(-\frac{2 i \dot{\phi}}{\mu}\right) \frac{1}{\left(1+|\phi|^{2}\right)^{2}}\left(\frac{2 i \dot{\phi}^{\dagger}}{\mu}\right)\right]
$$

\footnotetext{
${ }^{33} \mathrm{Up}$ to total derivatives, we have the local SUSY variation $\delta\left(L_{B}+L_{F}\right)=-i \dot{\epsilon} Q-i \dot{\epsilon}^{\dagger} Q^{\dagger}$.

${ }^{34}$ The quantum supercharges generate the SUSY transformation of any $\mathcal{O}=\mathcal{O}\left(\phi, \phi^{\dagger}, \psi, \psi^{\dagger}\right)$ via $\delta \mathcal{O}=$ $\left[\epsilon Q+\epsilon^{\dagger} Q^{\dagger}, \mathcal{O}\right]$. The relations (C.33) and (C.34) ensure that the SUSY transformations of a chiral superfield are correctly realized, or specifically that $\left[\epsilon Q+\epsilon^{\dagger} Q^{\dagger}, \psi\right]$ and $\left[\epsilon Q+\epsilon^{\dagger} Q^{\dagger}, \psi^{\dagger}\right]$ equate to $\delta \psi=-2 i \epsilon^{\dagger} \dot{\phi}$ and $\delta \psi^{\dagger}=2 i \epsilon \dot{\phi}^{\dagger}$, respectively.
} 
shows that $H_{\mathrm{cl}} \stackrel{q}{\rightarrow} H_{j+\chi^{\dagger} \chi-1 / 2}$. Further stipulating that

$$
\chi^{\dagger} \chi \stackrel{q}{\rightarrow} \chi^{\dagger} \chi-\left(\frac{1}{2}-\frac{1}{4 j}\right)
$$

reproduces precisely (C.37) from (C.36). In summary, the supersymmetric system $L_{B}+L_{F}$ in $S$ gauge can be quantized by applying the quantization rules (C.33), (C.34), (C.40) necessary to implement the supersymmetry algebra to the properly ordered classical expressions (C.35) and (C.39). ${ }^{35}$

Finally, judicious application of the aforementioned quantization rules ${ }^{36}$ shows that the classical expressions that, when quantized, give rise to the quantum operators $\left(L_{f}\right)_{i}$ are

$$
\begin{aligned}
\left(\vec{L}_{f}\right)_{\mathrm{cl}} & =\vec{V}+\frac{j+\chi^{\dagger} \chi-1 / 2}{1+|\phi|^{2}}\left(\phi+\phi^{\dagger},-i\left(\phi-\phi^{\dagger}\right),-\left(1-|\phi|^{2}\right)\right), \\
\vec{V} & \equiv \frac{\left(i\left(1-\phi^{2}\right) \dot{\phi}^{\dagger}-i\left(1-\left(\phi^{\dagger}\right)^{2}\right) \dot{\phi},-\left(1+\phi^{2}\right) \dot{\phi}^{\dagger}-\left(1+\left(\phi^{\dagger}\right)^{2}\right) \dot{\phi}, 2 i\left(\phi \dot{\phi}^{\dagger}-\phi^{\dagger} \dot{\phi}\right)\right)}{\mu\left(1+|\phi|^{2}\right)^{2}} .
\end{aligned}
$$

Classically, the bosonic angular momenta (C.21) take the form

$$
\left(L_{1}\right)_{\mathrm{cl}}=j\left(\frac{\phi+\phi^{\dagger}}{1+|\phi|^{2}}\right), \quad\left(L_{2}\right)_{\mathrm{cl}}=-i j\left(\frac{\phi-\phi^{\dagger}}{1+|\phi|^{2}}\right), \quad\left(L_{3}\right)_{\mathrm{cl}}=-j\left(\frac{1-|\phi|^{2}}{1+|\phi|^{2}}\right),
$$

so that $\left(\vec{L}_{f}\right)_{\mathrm{cl}}$ reduces to $\vec{L}_{\mathrm{cl}}$ with $j-1 / 2$ as $\mu \rightarrow \infty$ (note the differences in normalization and signs between the $J_{i}$ in $(4.16)$ and the $\left.\left(L_{i}\right)_{\mathrm{cl}}\right)$.

\section{Quantization of Chern-Simons theory}

Here, we review some basic aspects of the quantization of Chern-Simons theory with simple, compact $G$ that are relevant to our discussion in the main text, following [10, 21] (our conventions in section A.1 entail some differences in formulas relative to those references).

\section{D.1 Generalities}

Let $\Sigma$ be an oriented Riemann surface, to which canonical quantization on $\Sigma \times \mathbb{R}$ associates a Hilbert space $\mathcal{H}_{\Sigma}$. In temporal gauge $A_{0}=0$, the action (2.1) is $-\frac{k}{4 \pi} \int_{M^{3}} A_{1}^{a} \dot{A}_{2}^{a} d^{3} x$; we have the Poisson brackets and the (source-free) Gauss law constraint

$$
\left[A_{i}^{a}(x), A_{j}^{b}(y)\right]_{\mathrm{PB}}=\frac{4 \pi}{k} \epsilon_{i j} \delta^{a b} \delta^{(2)}(x-y), \quad \epsilon^{i j} F_{i j}^{a}=0 .
$$

If we impose the constraints before quantizing, then the physical phase space is the moduli space of flat connections on $\Sigma$, modulo gauge transformations: $\mathcal{M}=\operatorname{Hom}\left(\pi_{1}(\Sigma), G\right) / G$. $\mathcal{M}$ inherits a symplectic structure from the space of all connections $A_{i}^{a}(x)$ and has finite volume with respect to the symplectic volume element, so $\mathcal{H}_{\Sigma}$ is finite-dimensional.

\footnotetext{
${ }^{35}$ Again, these quantization rules do not fix the quantum representations of the canonical momenta $\pi_{\phi}$ and $\pi_{\phi^{\dagger}}$, but because we have chosen the boundary terms in the classical Lagrangian such that it is manifestly real, these representations are necessarily adjoints with respect to (C.32).

${ }^{36}$ Namely, after symmetrization of the form $f(\phi) \dot{\phi}^{\dagger}=\sqrt{f(\phi)} \dot{\phi}^{\dagger} \sqrt{f(\phi)}$ and $f\left(\phi^{\dagger}\right) \dot{\phi}=\sqrt{f\left(\phi^{\dagger}\right)} \dot{\phi} \sqrt{f\left(\phi^{\dagger}\right)}$.
} 
Choosing a complex structure $J$ on $\Sigma$ induces a Kähler structure on $\mathcal{M}$, after which $\mathcal{H}_{\Sigma}$ has the interpretation as the space of holomorphic sections of $\mathcal{L}^{k}$ where $\mathcal{L}$ is a suitable holomorphic line bundle over $\mathcal{M}$ (e.g., for $\mathrm{SU}(N)$, it is the determinant line bundle of the $\bar{\partial}$ operator on $\Sigma$ ). The symplectic form on $\mathcal{M}$ is the curvature of $\mathcal{L}^{k}$. One can show that the construction of $\mathcal{H}_{\Sigma}$ is canonically independent of $J$.

Here, we have assumed that $\partial \Sigma=0$, so that $\mathcal{H}_{\Sigma}$ is the finite-dimensional vector space of conformal blocks of the $\widehat{G}_{k}$ WZW model on $\Sigma$. If instead $\partial \Sigma \neq 0$, then $\mathcal{H}_{\Sigma}$ is an infinite-dimensional representation of the chiral algebra of the 2D CFT.

"Vertical" Wilson lines correspond to marked points $P_{i}$ on $\Sigma$ with an irreducible representation $R_{i}$ of $G$ associated to each. For $\mathcal{H}_{\Sigma ; P_{i}, R_{i}}$ to be nontrivial, all representations $R_{i}$ must correspond to integrable representations of $L G$. The appropriate reduced phase space $\mathcal{M}_{P_{i}, R_{i}}$ can be constructed with the aid of the Borel-Weil-Bott theorem.

This procedure can be phrased in the language of geometric quantization (see, e.g., [23]); we will not do so here. Let us instead make this abstract discussion concrete.

\section{D.2 Boundary conditions}

Suppose $\partial M^{3} \neq 0$. The variation of $(2.1)$ has both bulk and boundary components: ${ }^{37}$

$$
\delta S_{\mathrm{CS}}=\frac{k}{2 \pi} \int_{M^{3}} \operatorname{Tr}(\delta A F)+\frac{k}{4 \pi} \int_{\partial M^{3}} \operatorname{Tr}(\delta A A) .
$$

We will always choose boundary conditions such that there are no boundary corrections to the equations of motion. This can be achieved by setting one component of the gauge field to zero at the boundary: for instance, $A_{0}$ in the case of a spatial boundary (e.g., $M^{3}=\Sigma \times \mathbb{R}$ with $\partial \Sigma \neq 0$ and $\left.\partial M^{3}=\partial \Sigma \times \mathbb{R}\right)$ and either $A_{1}$ or $A_{2}$ in the case of a temporal boundary (e.g., $M^{3}=\Sigma \times(-\infty, 0]$ with $\partial \Sigma=0$ and $\left.\partial M^{3}=\Sigma\right)$. These two cases are suited to the "constrain, then quantize" and "quantize, then constrain" approaches, respectively.

When $M^{3}=\Sigma \times \mathbb{R}$, it is convenient to separate the temporal and spatial components of $d=d t \partial_{t}+\tilde{d}$ and $A=A_{0}+\tilde{A}$, giving

$$
S_{\mathrm{CS}}=-\frac{k}{4 \pi} \int_{M^{3}} \operatorname{Tr}\left(\tilde{A} \partial_{t} \tilde{A}\right) d t+\frac{k}{2 \pi} \int_{M^{3}} \operatorname{Tr}\left(A_{0} \tilde{F}\right)+\frac{k}{4 \pi} \int_{\partial M^{3}} \operatorname{Tr}\left(A_{0} \tilde{A}\right)
$$

where $\tilde{F}=\tilde{d} \tilde{A}-i \tilde{A}^{2}$. If $\partial \Sigma \neq 0$, then we impose $\left.A_{0}\right|_{\partial M^{3}}=0$, which kills the boundary term in (D.3). This boundary condition implies that gauge transformations independent of time on the boundary are global (because, in an alternate quantization, they act nontrivially on the wavefunctions of physical states), while only those that reduce to the identity on the boundary are truly gauge. Further integrating out $A_{0}$ in (D.3) enforces $\tilde{F}=0$, and we arrive at an effective action for $\tilde{A}$ satisfying this constraint by substituting such $\tilde{A}$ into the first term of (D.3) (gauge-equivalent choices of $\tilde{A}$ yield the same action).

\footnotetext{
${ }^{37}$ In coordinates, the variation of $\mathcal{L}_{\mathrm{CS}}$ in $(\mathrm{A} .1)$ is $\delta \mathcal{L}_{\mathrm{CS}}=\epsilon^{\mu \nu \rho} \operatorname{Tr}\left(\delta A_{\mu} F_{\nu \rho}\right)+\partial_{\nu}\left[\epsilon^{\mu \nu \rho} \operatorname{Tr}\left(A_{\mu} \delta A_{\rho}\right)\right]$. For an infinitesimal gauge transformation, $\delta A_{\mu}^{a}=D_{\mu} \theta^{a}$ and both terms reduce to boundary terms, which combine to give $\delta \mathcal{L}_{\mathrm{CS}}=\frac{1}{2} \epsilon^{\mu \nu \rho} \partial_{\nu}\left(A_{\rho}^{a} \partial_{\mu} \theta^{a}\right)$. This is indeed the infinitesimal form of $-i \epsilon^{\mu \nu \rho} \partial_{\mu} \operatorname{Tr}\left(A_{\nu} g^{-1} \partial_{\rho} g\right)$ from (A.2) (for an infinitesimal gauge transformation, the Pontryagin density term does not contribute).
} 
When $\partial M^{3}=\Sigma$, it is natural to compute wavefunctions in the path integral formalism. In the gauge $A_{0}=0$, the phase space coordinates are the two components of $\tilde{A}$ in the $\Sigma$ direction, one of which represents the canonical coordinate on which the wavefunction(al) depends. ${ }^{38}$ Specifying nonzero values of $A_{1}$ on the boundary requires adding a term

$$
\frac{k}{4 \pi} \int_{\partial M^{3}} \operatorname{Tr}\left(A_{1} A_{2}\right)
$$

to (2.1) so that the boundary term in the variation of the total action is $\frac{k}{2 \pi} \int_{\partial M^{3}} \operatorname{Tr}\left(\delta A_{1} A_{2}\right)$, which vanishes because $\left.\delta A_{1}\right|_{\partial M^{3}}=0$. Specifying $A_{2}$ requires a term of the opposite sign as in (D.4). Aside from ensuring no boundary corrections to the equations of motion, the necessity of the boundary action (D.4) follows from consistency of the canonical formalism in which $A_{1}, A_{2}$ are conjugate variables $q, p$ : it is precisely the surface term that, when added to $\left.S_{\mathrm{CS}}\right|_{A_{0}=0}$, brings the action to the standard form $\propto \int p \dot{q}$.

\section{D.3 Real polarization}

In this subsection, we take $M^{3}=\Sigma \times \mathbb{R}$.

\section{D.3.1 $\Sigma=D^{2}$}

Because $D^{2}$ is simply connected, as is $G$, the flatness constraint $\tilde{F}=0$ is solved by

$$
\tilde{A}=-i \tilde{d} U U^{-1}
$$

where $U: M^{3} \rightarrow G$ is single-valued. The change of variables $D \tilde{A} \delta(\tilde{F}) \rightarrow D U$ in the path integral incurs no Jacobian [21]. Setting $\left.A_{0}\right|_{\partial M^{3}}=0$, the effective action (D.3) when written in terms of $U$ is

$$
S_{\text {eff }}=k S_{C}^{+}(U) \equiv-\frac{k}{4 \pi} \int_{\partial M^{3}} \operatorname{Tr}\left(U^{-1} \partial_{\phi} U U^{-1} \partial_{t} U\right) d \phi d t-\frac{k}{12 \pi} \int_{M^{3}} \operatorname{Tr}\left(U^{-1} d U\right)^{3}
$$

where $\phi$ denotes the angular coordinate on $D^{2}$ and the chiral WZW (CWZW) action $S_{C}^{+}(U)$ depends only on the boundary values of $U$. The action (D.6) is invariant under the following transformation on $\partial M^{3}$ :

$$
U(\phi, t) \rightarrow \tilde{V}(\phi) U(\phi, t) V(t) .
$$

Since $\tilde{V}$ is a global symmetry, the Hilbert space is a representation of the loop group $L G$. On the other hand, $V$ is a gauge symmetry. The classical phase space is then $L G / G$, where $L G$ is the space of flat $G$-connections on $D^{2}$ modulo gauge transformations that reduce to the identity on $\partial D^{2}=S^{1}$ and the quotient by $G$ fixes the $V$ gauge symmetry. This space inherits a symplectic structure from the gauge-fixed Lagrangian [82].

Our primary interest is in the case where the $D^{2}$ contains a source in the representation $\lambda$. Its effect is modeled by adding to (D.3) the coadjoint orbit action

$$
i \int_{\mathbb{R}} \operatorname{Tr}\left(\lambda g^{-1}\left(\partial_{t}-i A_{t}\right) g\right) d t=\frac{i}{2 \pi} \int_{\partial M^{3}} \operatorname{Tr}\left(\lambda g^{-1}\left(\partial_{t}-i A_{t}\right) g\right) d \phi d t
$$

\footnotetext{
${ }^{38}$ Here, we have in mind a real polarization where the canonical coordinate is, e.g., $A_{1}$ or $A_{2}$ rather than $A_{z}$; we will discuss the holomorphic polarization later.
} 
where $g(t) \in G$ and $\lambda$ (which fixes a maximal torus $T \subset G$ ) is written in a basis of Cartan generators. As discussed in section 3.2, the gauge invariance of (D.8) under $g(t) \rightarrow g(t) h(t)$ with $h(t) \in T$ suffers from global anomalies unless $\lambda$ is quantized as a weight. ${ }^{39}$ By itself, (D.8) describes the quantum mechanics of $g$ coupled to the background gauge field $A_{t}$, with classical phase space $G / T$ and symplectic structure $\operatorname{Tr}\left(\lambda\left(g^{-1} \delta g\right)^{2}\right)$. Putting the source at the origin and integrating over $A_{t}$ in the total action (D.3) plus (D.8) yields the constraint

$$
\frac{k}{2 \pi} \tilde{F}(x, t)+g(t) \lambda g^{-1}(t) \delta^{(2)}(x) d^{2} x=0 .
$$

At any given $t$, integrating (D.9) over a disk containing the origin immediately shows that the logarithm of the holonomy of the flat connection $\tilde{A}$ around the source is $-\frac{2 \pi i}{k} g(t) \lambda g^{-1}(t)$. In other words, the conjugacy class of the holonomy of $\tilde{A}$ is determined by $\lambda$ to be that of $e^{-2 \pi i \lambda / k}$. Explicitly, (D.9) is solved by

$$
\tilde{A}=-i \tilde{d} \tilde{U} \tilde{U}^{-1}, \quad \tilde{U} \equiv U \exp \left[\frac{i}{k} g(t) \lambda g^{-1}(t) \phi\right]
$$

where at any given $t, U$ is single-valued on $D^{2}$ and its value at the origin $U(0, t)$ commutes with $g(t) \lambda g^{-1}(t)$. Substituting (D.10) into the total action (D.3) plus (D.8) and integrating out $g$, whose equation of motion imposes $[\lambda, g]=0$, yields the effective action

$$
S_{\text {eff }}=k S_{C}^{+}(U)-\frac{i}{2 \pi} \int_{\partial M^{3}} \operatorname{Tr}\left(\lambda U^{-1} \partial_{t} U\right) d \phi d t
$$

The action (D.11) is now invariant under (D.7) where $V$ commutes with $\lambda$, so the classical phase space is $L G / T$. The Hilbert space $\mathcal{H}_{\Sigma}$ is the integrable representation $\mathcal{H}_{\lambda}$ of $\widehat{G}_{k}$.

\section{D.3.2 $\Sigma=T^{2}$}

For $G$ connected and simply connected, the most general flat connection on $T^{2}$ is

$$
\tilde{A}=-i \tilde{d} U U^{-1}+U \theta(t) U^{-1}
$$

where $U$ is single-valued and $\theta$ is a $\mathfrak{g}$-valued one-form representing the holonomies. Since $\pi_{1}\left(T^{2}\right)$ is abelian, the two components of $\theta$ can be chosen to lie in a Cartan subalgebra $\mathfrak{t}$ with basis $\vec{H}: \theta=\vec{\theta} \cdot \vec{H}$. This description suffers from a gauge redundancy $\vec{\theta} \sim \vec{\theta}+2 \pi \vec{\alpha}$ where $\vec{\alpha}$ is a one-form valued in $\Lambda_{R}^{\vee}$. Hence the classical phase space is

$$
\mathcal{M}=\frac{T \times T}{\mathcal{W}}
$$

where $T$ is a maximal torus. Substituting (D.12) into (D.3) and using that the change of variables $D \tilde{A} \delta(\tilde{F}) \rightarrow D U D \theta_{1} D \theta_{2}$ entails no Jacobian [21] gives the following effective action for $\theta_{1,2}$, which are naturally interpreted as canonical coordinates and momenta:

$$
S_{\mathrm{eff}}=-\frac{k}{2 \pi} \int_{M^{3}} \overrightarrow{\theta_{1}} \cdot \dot{\vec{\theta}}_{2} d t \Longrightarrow\left[\theta_{1}^{i}, \theta_{2}^{j}\right]=\frac{2 \pi i}{k} \delta^{i j} .
$$

\footnotetext{
${ }^{39} \mathrm{~A}$ weight $\lambda$ of $G$ is integral if for each $t \in \mathfrak{t} \operatorname{such}$ that $\exp (i t)=1 \in G, \lambda(t) \in 2 \pi \mathbb{Z}$; for $G$ semisimple, the integral weights comprise a sublattice of the weight lattice, but these lattices coincide if $G$ is simply connected.
} 
Since the coordinates $\theta_{1}$ are compact, the momentum is quantized and momentum eigenstates are labeled by $\vec{\lambda}$ in the weight lattice $\Lambda_{W}$ of $G$. The momenta $\theta_{2}$ are also compact by virtue of the aforementioned gauge redundancy. Finally, the action of $\mathcal{W}$ leads to a further redundancy $\vec{\theta} \sim \mathcal{W}(\vec{\theta})$. Thus we deduce that the Hilbert space in genus one is

$$
\mathcal{H}_{\Sigma} \cong \frac{\Lambda_{W}}{\mathcal{W} \ltimes k \Lambda_{R}^{\vee}}
$$

where $\Lambda_{R}^{\vee} \cong \Lambda_{R}$ for simply laced $G$ (using our normalization conventions). This coset space is precisely the space of integrable representations of $\widehat{G}_{k}\left(\mathcal{W} \ltimes \Lambda_{R}^{\vee}\right.$ is the affine Weyl group).

\section{D.4 Holomorphic polarization}

In this scheme, we first quantize and then constrain: the classical phase space is the infinitedimensional space of all gauge fields $\mathcal{A}$ rather than the moduli space of flat connections $\mathcal{M}$. Wavefunctions are derived by demanding that they satisfy Gauss's law rather than by evaluating the path integral. This approach has the virtue of making explicit the identification of wavefunctions and conformal blocks of the corresponding 2D CFT on any $\Sigma$.

\section{D.4.1 Coherent states}

In holomorphic quantization, we separate out the holomorphic part of the wavefunction and regard the non-holomorphic part (namely, the exponentiated Kähler potential on $\mathcal{A}$ induced by a choice of complex structure on $\Sigma$ ) as part of the integration measure. Coherent states furnish a resolution of the identity with respect to this measure.

In the gauge $A_{0}=0$, and having fixed a complex structure on $\Sigma$, we obtain from (D.1) the canonical commutation relations

$$
\left[A_{z}^{a}\left(z_{1}\right), A_{\bar{z}}^{b}\left(z_{2}\right)\right]=\frac{2 \pi i}{k} \delta^{a b} \delta^{(2)}\left(z_{1}-z_{2}\right)
$$

where $A_{z}^{a}=\frac{1}{2}\left(A_{1}^{a}-i A_{2}^{a}\right)$. Wavefunctions are holomorphic in $A_{z}$, with the action of $A_{z}^{a}$ and $A_{\bar{z}}^{a}$ being represented thereon by

$$
\mathbf{A}_{z}^{a}=A_{z}^{a}, \quad \mathbf{A}_{\bar{z}}^{a}=-\frac{2 \pi i}{k} \frac{\delta}{\delta A_{z}^{a}}
$$

The coherent state inner product is defined as

$$
\left\langle\Psi_{1} \mid \Psi_{2}\right\rangle=\int D A_{z}^{a}(x) D A_{z}^{a}(x)^{*} e^{\frac{i k}{\pi} \int_{\Sigma} d^{2} z \operatorname{Tr}\left(A_{z} A_{z}^{*}\right)} \Psi_{1}\left[A_{z}\right]^{*} \Psi_{2}\left[A_{z}\right]
$$

on the infinite-dimensional space of functionals of $A_{z}$, of which the physical Hilbert space $\mathcal{H}_{\Sigma}$ is the gauge-invariant subspace.

The following formulas are useful in constructing the physical wavefunctions. We define the WZW actions

$$
S^{ \pm}[g] \equiv-\frac{1}{4 \pi} \int_{\Sigma} d^{2} z \operatorname{Tr}\left(g^{-1} \partial_{z} g g^{-1} \partial_{\bar{z}} g\right) \mp \frac{1}{12 \pi} \int_{M^{3}} \operatorname{Tr}\left(g^{-1} d g\right)^{3},
$$


where $\partial M^{3}=\Sigma$. The corresponding Polyakov-Wiegmann identities are

$$
S^{ \pm}\left[g_{1} g_{2}\right]=S^{ \pm}\left[g_{1}\right]+S^{ \pm}\left[g_{2}\right]-\frac{1}{2 \pi} \int_{\Sigma} d^{2} z \begin{cases}\operatorname{Tr}\left(\partial_{z} g_{2} g_{2}^{-1} g_{1}^{-1} \partial_{\bar{z}} g_{1}\right) & (+), \\ \operatorname{Tr}\left(g_{1}^{-1} \partial_{z} g_{1} \partial_{\bar{z}} g_{2} g_{2}^{-1}\right) & (-),\end{cases}
$$

from which we read off the variations

$$
\delta S^{ \pm}[g]=\frac{1}{2 \pi} \int_{\Sigma} d^{2} z \begin{cases}\operatorname{Tr}\left(g^{-1} \delta g \partial_{z}\left(g^{-1} \partial_{\bar{z}} g\right)\right) & (+), \\ \operatorname{Tr}\left(g^{-1} \delta g \partial_{\bar{z}}\left(g^{-1} \partial_{z} g\right)\right) & (-) .\end{cases}
$$

To begin, consider $\Sigma=S^{2}$. For all $A_{z}$ (not necessarily flat) except in a subset of codimension one, it is possible to write

$$
A_{z}=-i \partial_{z} U U^{-1}
$$

where $U: S^{2} \rightarrow G_{\mathbb{C}}[21,69]$. The unique physical state on $S^{2}$ has wavefunction

$$
\Psi_{0}\left[A_{z}\right]=e^{2 i k S^{-}[U]},
$$

up to normalization. Indeed, we have by (D.21) and (D.17) that

$$
\delta \Psi_{0}\left[A_{z}\right]=\frac{k}{\pi}\left[\int_{\Sigma} d^{2} z \operatorname{Tr}\left(\delta A_{z} \partial_{\bar{z}} U U^{-1}\right)\right] \Psi_{0}\left[A_{z}\right] \Longrightarrow \mathbf{A}_{\bar{z}}^{a} \Psi_{0}\left[A_{z}\right]=-i\left(\partial_{\bar{z}} U U^{-1}\right)^{a} \Psi_{0}\left[A_{z}\right]
$$

(recall the conventions in section A.1). Again in light of (D.17), the Gauss law constraint $\mathbf{F}_{z \bar{z}} \Psi_{0}\left[A_{z}\right]=0$ is satisfied. Under $A_{z} \rightarrow A_{z}^{g}$ (i.e., $\left.U \rightarrow g U\right), \Psi_{0}\left[A_{z}\right]$ transforms by a phase (1-cocycle), which motivates us to represent the gauge transformation $g(x)$ by an operator $\mathbf{U}(g)$ that acts on arbitrary functionals as

$$
\mathbf{U}(g) \Psi\left[A_{z}\right]=e^{-2 i k S^{+}[g]-\frac{k}{\pi} \int_{\Sigma} d^{2} z \operatorname{Tr}\left(A_{z} g^{-1} \partial_{\bar{z}} g\right)} \Psi\left[A_{z}^{g}\right] .
$$

By (D.20), we deduce that $\mathbf{U}(g) \Psi_{0}\left[A_{z}\right]=\Psi_{0}\left[A_{z}\right]$, which is a restatement of the fact that the physical wavefunction is gauge-invariant. $\mathbf{U}(g)$ is unitary with respect to the inner product (D.18) and, by (D.20), satisfies the composition law $\mathbf{U}\left(g_{2}\right) \mathbf{U}\left(g_{1}\right)=\mathbf{U}\left(g_{1} g_{2}\right)$.

\section{D.4.2 $\Sigma=T^{2}$ redux}

The Hodge decomposition in this case (analogous to (D.22) on $S^{2}$ ), which holds for almost all $A_{z}$, is

$$
A_{z}=-i \partial_{z} U U^{-1}+U a_{z} U^{-1}
$$

where $U \in G_{\mathbb{C}}$ and $a_{z}$ lies in a fixed Cartan subalgebra $\mathfrak{t} \subset \mathfrak{g}$ (more precisely, $\mathfrak{t}_{\mathbb{C}}$ ). Gauge invariance with respect to (D.24) restricts physical wavefunctions, which we preemptively label by a subscript $\lambda$, to take the form

$$
\Psi_{\lambda}\left[A_{z}\right]=e^{2 i k S^{-}[U]+\frac{k}{\pi} \int_{\Sigma} d^{2} z \operatorname{Tr}\left(a_{z} U^{-1} \partial_{\bar{z}} U\right)} \psi_{\lambda}\left(a_{z}\right) .
$$

Using this parametrization in (D.18), changing variables from $A_{z}$ to $\left(U, a_{z}\right)$ (which involves a nontrivial Jacobian), and integrating over $U$ leads to an effective quantum mechanics with coherent state inner product

$$
\left\langle\psi_{\lambda_{1}} \mid \psi_{\lambda_{2}}\right\rangle=\int d a_{z} d a_{z}^{*} e^{\frac{i(k+h)}{\pi} \int_{\Sigma} d^{2} z \operatorname{Tr}\left(a_{z} a_{z}^{*}\right)} \psi_{\lambda_{1}}^{\mathrm{eff}}\left(a_{z}\right)^{*} \psi_{\lambda_{2}}^{\mathrm{eff}}\left(a_{z}\right)
$$


The effective wavefunctions $\psi_{\lambda}^{\text {eff }}\left(a_{z}\right)$ are related to the $\psi_{\lambda}\left(a_{z}\right)$ in (D.26) by

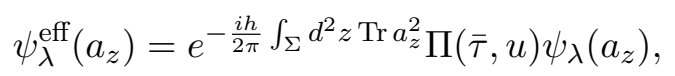

where $\tau$ denotes the complex structure and $u \equiv-\frac{\operatorname{Im} \tau}{\pi} a_{z}$ as in (6.55). In particular, for the $\psi_{\lambda}\left(a_{z}\right)$ in (6.55), the corresponding effective wavefunctions

$$
\psi_{\lambda}^{\text {eff }}\left(a_{z}\right)=e^{-\frac{(k+h) \operatorname{Im} \tau}{\pi}} \operatorname{Tr} a_{z}^{2} \Theta_{\lambda+\rho, k+h}^{-}(\bar{\tau}, u)
$$

are orthogonal with respect to (D.27): $\left\langle\psi_{\lambda_{1}} \mid \psi_{\lambda_{2}}\right\rangle \propto \delta_{\lambda_{1} \lambda_{2}}$. In writing (D.29), we have used

$$
\Pi(\tau, u) \chi_{\lambda}^{(k)}(\tau, u)=\Theta_{\lambda+\rho, k+h}^{-}(\tau, u)
$$

and the same conventions for the geometry of $\Sigma=T^{2}$ as [21], in which the area is $-2 i \operatorname{Im} \tau$; the functional determinant $\Pi$ and the Weyl-odd theta functions $\Theta^{-}$are defined in [21] and references therein (in comparing Lie algebra conventions, note that we use $\operatorname{Tr}\left(T^{a} T^{b}\right)=$ $\frac{1}{2} \delta^{a b},[21]$ uses $\operatorname{Tr}\left(T^{a} T^{b}\right)=-\delta^{a b}$, and [69] uses $\left.\operatorname{Tr}\left(T^{a} T^{b}\right)=-\frac{1}{2} \delta^{a b}\right)$.

The key point is the following. Level- $k$ theta functions for $\Lambda_{R}$ are indexed by weights in $\Lambda_{W} / k \Lambda_{R}$ and transform by phases under large gauge transformations, which act as $u \rightarrow u+r_{1}+\tau r_{2}$ for $r_{1,2} \in \Lambda_{R}$ (hence the integration region for $a_{z}$ in (D.27) is the complex torus $\left.T_{\mathbb{C}}=\mathfrak{t}_{\mathbb{C}} / \Lambda_{R}\right)$. The Weyl-invariant subspace of such theta functions is spanned by Weyl-Kac characters at level $k$. However, Weyl-Kac characters at $k, \lambda$ are expressible in terms of theta functions at $k+h, \lambda+\rho$. Therefore, rather than labeling the spectrum by $\lambda$ in the Weyl alcove $\Lambda_{W} /\left(\mathcal{W} \ltimes k \Lambda_{R}\right)$, one may equivalently label it by $\lambda$ in the interior of the dilated Weyl alcove $\Lambda_{W} /\left(\mathcal{W} \ltimes(k+h) \Lambda_{R}\right)$. While this statement is familiar from representation theory [83], the relation between (6.55) and (D.29) gives it a physical interpretation.

\section{E Surgery versus localization}

It is amusing, and possibly even useful, that localization offers an alternative to traditional algebraic or surgery-based methods for the computation of certain knot invariants. Let us compare localization for $\mathcal{N}=2 \mathrm{SU}(2)_{k+2}$ and surgery for $\mathcal{N}=0 \mathrm{SU}(2)_{k}$ on $S^{3}$ in a few examples. The Chern-Simons observables that are accessible to localization on $S^{3}$ include links composed of Hopf fibers: their components are unknots with pairwise linking number one. For such links, one can check that the matrix model results match those from surgery for small numbers of components or sufficiently small representations, but it is possible to obtain clean answers using localization even when the latter method becomes cumbersome.

The localization approach is as follows. For $\mathcal{N}=2 \mathrm{SU}(2)_{k+2}$ with Cartan parametrized by $\operatorname{diag}(a,-a)$, the matrix model of [15], written in (6.37), reduces to

$$
\left\langle W_{j_{1}} \cdots W_{j_{n}}\right\rangle_{\mathcal{N}=2}=-\frac{2}{Z_{\mathcal{N}=2}} \int_{\mathbb{R}} d a e^{-2 \pi i(k+2-i \epsilon) a^{2}} \sinh (2 \pi a)^{2} \operatorname{Tr}_{j_{1}}\left(e^{2 \pi a}\right) \cdots \operatorname{Tr}_{j_{n}}\left(e^{2 \pi a}\right)
$$


where $Z_{\mathcal{N}=2}=e^{i \pi / 4} e^{-i \pi /(k+2)} Z_{\mathcal{N}=0}$ with $Z_{\mathcal{N}=0}$ in (2.6). It is useful to define two equivalent expressions for $\operatorname{Tr}_{j}\left(e^{2 \pi a}\right)$, both before and after applying the Weyl character formula:

$$
\operatorname{Tr}_{j}^{\exp }\left(e^{2 \pi a}\right) \equiv \sum_{m=-j}^{j} e^{4 \pi m a}, \quad \operatorname{Tr}_{j}^{\sinh }\left(e^{2 \pi a}\right) \equiv \frac{\sinh (2 \pi a(2 j+1))}{\sinh (2 \pi a)} .
$$

Note that we label representations by their spin, not by their dimension. Results are conveniently written in terms of $q \equiv e^{2 \pi i /(k+2)}$ and the quadratic Casimirs $C_{2}(j) \equiv j(j+1)$ of the representations. The supersymmetric framing in the $\mathcal{N}=2 \mathrm{SU}(2)_{k+2}$ theory leads to a phase of $q^{-C_{2}(j)}$ for each Wilson line relative to the $\mathcal{N}=0 \mathrm{SU}(2)_{k}$ theory:

$$
\left\langle W_{j_{1}} \cdots W_{j_{n}}\right\rangle_{\mathcal{N}=2}=q^{-C_{2}\left(j_{1}\right)-\cdots-C_{2}\left(j_{n}\right)}\left\langle W_{j_{1}} \cdots W_{j_{n}}\right\rangle_{\mathcal{N}=0} .
$$

From the integration measure in (E.1), we see that although the integrand can always be written as a sum of Gaussians by inserting $\operatorname{Tr}_{j}^{\exp }\left(e^{2 \pi a}\right)$, correlators of two or fewer Wilson loops are particularly simple because each insertion of $\operatorname{Tr}_{j}^{\sinh }\left(e^{2 \pi a}\right)$ cancels a factor of $\sinh (2 \pi a)$. These are precisely the cases where the results have simple expressions in terms of the $S$-matrix elements (2.11).

To describe the surgery approach, we follow [68], where our $q$ is denoted there by $q^{-1}$. A link with $n$ components is conveniently regarded as the closure of a braid on $\geq n$ strands. ${ }^{40}$ Given a link with specified representations for its components, we write its expectation value \langle\rangle$_{\mathcal{N}=0}$ in the $\mathcal{N}=0 \mathrm{SU}(2)_{k}$ theory as a braid group element enclosed in brackets \langle\rangle$_{\#}$, where \# denotes the number of strands (if all components are in the same representation, then the brackets \langle\rangle$_{\#}$ are cyclic). Strands are labeled from left to right, and braid moves are applied from bottom to top. The braid group generator $g_{k}$ corresponds to crossing strand $k$ over strand $k+1$, and the braid group relations are

$$
g_{i} g_{i+1} g_{i}=g_{i+1} g_{i} g_{i+1}, \quad g_{i} g_{j}=g_{j} g_{i}(|i-j| \geq 2) .
$$

Canonical framing is assumed, which means that each unit of writhe (self-intersection number) introduces a factor of $q^{-C_{2}(j)}$ for a line of spin $j$, where self-overcross corresponds to positive writhe. The basic properties that allow us to compute link expectation values are the fusion property (OPE) for cabled unknots, ${ }^{41}$

$$
\left\langle W_{\rho_{1}} W_{\rho_{2}} \cdots\right\rangle_{\mathcal{N}=0}=\left\langle W_{\rho_{1} \otimes \rho_{2}} \cdots\right\rangle_{\mathcal{N}=0}=\sum_{\rho \in \rho_{1} \otimes \rho_{2}}\left\langle W_{\rho} \cdots\right\rangle_{\mathcal{N}=0},
$$

and similar fusion properties for crossed lines (derivable from the braiding matrix), which hold for lines in arbitrary representations of any $G$. Using these properties, one can derive inductively the results for the unknot and Hopf link in arbitrary representations of $\mathrm{SU}(2)$ :

$$
\begin{gathered}
\left\langle W_{j}\right\rangle_{\mathcal{N}=0}=\langle 1\rangle_{1}=\frac{q^{(2 j+1) / 2}-q^{-(2 j+1) / 2}}{q^{1 / 2}-q^{-1 / 2}}, \\
\left\langle W_{j_{1}} W_{j_{2}}\right\rangle_{\mathcal{N}=0}=\left\langle g_{1}^{2}\right\rangle_{2}=\frac{q^{\left(2 j_{1}+1\right)\left(2 j_{2}+1\right) / 2}-q^{-\left(2 j_{1}+1\right)\left(2 j_{2}+1\right) / 2}}{q^{1 / 2}-q^{-1 / 2}} .
\end{gathered}
$$

\footnotetext{
${ }^{40}$ The following is a technical simplification of the original procedure of [10], which involves passing from $S^{2} \times S^{1}$ to $S^{3}$ via braid traces with a "spectator" strand.

${ }^{41}$ From (6.37), BPS Wilson loops in the $\mathcal{N}=2$ theory manifestly satisfy this property as well [55].
} 
Let us, however, restrict our attention to lines in the fundamental of $\mathrm{SU}(2)$ (the situation relevant to the Jones polynomial). For such lines, the basic fusion properties (whose explicit forms we will not need) imply the familiar skein relation

$$
q^{-1 / 4} L_{+}-q^{1 / 4} L_{-}=\left(q^{-1 / 2}-q^{1 / 2}\right) L_{0}
$$

where $L_{+}, L_{-}$, and $L_{0}$ denote overcross, undercross, and no cross, respectively. For fundamental lines, the skein relation (E.8), the writhe relations (factors of $q^{\mp 3 / 4}$ for each self-overcross and self-undercross), and the result $\left\langle W_{1 / 2}\right\rangle_{\mathcal{N}=0}$ for the unknot suffice to determine all link expectation values [68]. Moreover, using (E.8) rather than fusion allows us to consider only fundamental lines at all intermediate steps in the computation.

As an example of the use of (E.8), we compute for the fundamental trefoil knot that

$$
\left\langle g_{1}^{3}\right\rangle_{2}=q^{-7 / 4}\left(1+q+q^{2}-q^{4}\right) .
$$

As a more relevant class of examples, consider $W_{1 / 2}^{n}$ where all components are understood to lie along Hopf fibers. We may construct the corresponding braid on $n$ strands by linking the first with the rest, the second with the remainder, and so on:

$$
\left\langle W_{1 / 2}^{n}\right\rangle_{\mathcal{N}=0}=\left\langle\left(g_{n-1}^{2}\right) \cdots\left(g_{2} \cdots g_{n-2} g_{n-1}^{2} g_{n-2} \cdots g_{2}\right)\left(g_{1} \cdots g_{n-2} g_{n-1}^{2} g_{n-2} \cdots g_{1}\right)\right\rangle_{n} .
$$

Special cases are

$$
\begin{aligned}
& \left\langle W_{1 / 2}\right\rangle_{\mathcal{N}=0}=q^{1 / 2}+q^{-1 / 2}, \\
& \left\langle W_{1 / 2}^{2}\right\rangle_{\mathcal{N}=0}=q^{3 / 2}+q^{1 / 2}+q^{-1 / 2}+q^{-3 / 2}, \\
& \left\langle W_{1 / 2}^{3}\right\rangle_{\mathcal{N}=0}=q^{-3}+q^{-2}+q^{-1}+1+2 q+2 q^{2},
\end{aligned}
$$

where the first two expressions follow from (E.6) and (E.7) and the last expression is computed from $\left\langle W_{1 / 2}^{3}\right\rangle_{\mathcal{N}=0}=\left\langle g_{2}^{2} g_{1} g_{2}^{2} g_{1}\right\rangle_{3}$ using (E.8), cyclicity of \langle\rangle$_{\#}$, and the first of the braid group relations (E.4). Note that when $n \geq 3$, the result for $\left\langle W_{1 / 2}^{n}\right\rangle_{\mathcal{N}=0}$ is not invariant under $q \leftrightarrow q^{-1}$. An inductive argument might suffice to compute $\left\langle W_{1 / 2}^{n}\right\rangle_{\mathcal{N}=0}$ via surgery, or a representation-theoretic point of view might prove more useful, as in the case of torus knots (see [14] and references therein). Regardless, by inserting $\operatorname{Tr}_{1 / 2}^{\exp }\left(e^{2 \pi a}\right)^{n}$ into the localization matrix model (E.1), we compute with almost no effort that

$$
\left\langle W_{1 / 2}^{n}\right\rangle_{\mathcal{N}=2}=\frac{1}{2(1-q)} \sum_{\ell=0}^{n}\left(\begin{array}{l}
n \\
\ell
\end{array}\right) q^{-(n-2 \ell)^{2} / 4}\left(q^{n-2 \ell}+q^{-(n-2 \ell)}-2 q\right) .
$$

Accounting for the framing discrepancy (E.3), we deduce that

$$
\left\langle W_{1 / 2}^{n}\right\rangle_{\mathcal{N}=2}=q^{-3 n / 4}\left\langle W_{1 / 2}^{n}\right\rangle_{\mathcal{N}=0},
$$

from which we read off the Jones polynomial of all links whose components are Hopf fibers. ${ }^{42}$

\footnotetext{
${ }^{42}$ To get the Jones polynomial from SU(2) Chern-Simons with fundamental Wilson loops, we must divide by the expectation value of the unknot, adjust by an overall power of $q$, and redefine $q$ slightly [68]:

$$
\frac{q^{3 w(L) / 4}}{q^{1 / 2}+q^{-1 / 2}}\langle L\rangle \stackrel{q^{1 / 2} \rightarrow-q^{-1 / 2}}{\longrightarrow} V(L)
$$

where $w(L)$ is the writhe of the link $L$. For example, for the unknot $(w=0)$, Hopf link $(w=+2)$, and trefoil $(w=+3)$, we get $1,-q^{-5 / 2}-q^{-1 / 2}$, and $-q^{-4}+q^{-3}+q^{-1}$, respectively. This is a Laurent polynomial in $q^{1 / 2}$ (or, if the link has an odd number of components, in $q$ ).
} 
Finally, one can consider more general $\mathrm{SU}(2)$ representations. For example, the expectation value of a three-component Hopf link with each component in an arbitrary representation is easily computed via localization by inserting $\operatorname{Tr}_{J_{1}, J_{2}}^{\sinh }\left(e^{2 \pi a}\right)$ and $\operatorname{Tr}_{J_{3}}^{\exp }\left(e^{2 \pi a}\right)$ into (E.1):

$$
\left\langle W_{J_{1}} W_{J_{2}} W_{J_{3}}\right\rangle_{\mathcal{N}=2}=\frac{1}{1-q^{-1}} \sum_{\ell=-J_{3}}^{J_{3}}\left(q^{-\left(J_{1}-J_{2}+\ell\right)^{2}}-q^{-\left(1+J_{1}+J_{2}+\ell\right)^{2}}\right) .
$$

This expression is invariant under permutations of $\left\{J_{1}, J_{2}, J_{3}\right\}$. To compare to surgery, one might hope to use a generalized skein relation, which is a linear relation between $N+1$ crossings where $N$ is the number of irreducible representations in the decomposition of the tensor product of two lines. However, skein relations only make sense for all lines in the same representation, and only for the fundamental do they alone suffice to determine knot invariant polynomials [68]. Hence deriving this result using surgery would require appealing to the underlying fusion properties, which is arguably more complicated than evaluating a one-dimensional Gaussian integral.

Open Access. This article is distributed under the terms of the Creative Commons Attribution License (CC-BY 4.0), which permits any use, distribution and reproduction in any medium, provided the original author(s) and source are credited.

\section{References}

[1] D. Birmingham, M. Blau, M. Rakowski and G. Thompson, Topological field theory, Phys. Rept. 209 (1991) 129 [INSPIRE].

[2] J. Kallen, Cohomological localization of Chern-Simons theory, JHEP 08 (2011) 008 [arXiv: 1104.5353] [INSPIRE].

[3] W. Chen, G.W. Semenoff and Y.-S. Wu, Two loop analysis of nonAbelian Chern-Simons theory, Phys. Rev. D 46 (1992) 5521 [hep-th/9209005] [INSPIRE].

[4] E. Witten, Supersymmetric index of three-dimensional gauge theory, hep-th/9903005 [INSPIRE].

[5] S.R. Coleman and B.R. Hill, No More Corrections to the Topological Mass Term in QED in Three-Dimensions, Phys. Lett. B 159 (1985) 184 [inSPIRE].

[6] C. Closset, T.T. Dumitrescu, G. Festuccia, Z. Komargodski and N. Seiberg, Contact Terms, Unitarity and F-Maximization in Three-Dimensional Superconformal Theories, JHEP 10 (2012) 053 [arXiv:1205.4142] [INSPIRE].

[7] C. Closset, T.T. Dumitrescu, G. Festuccia, Z. Komargodski and N. Seiberg, Comments on Chern-Simons Contact Terms in Three Dimensions, JHEP 09 (2012) 091 [arXiv: 1206.5218] [INSPIRE].

[8] R.J. Szabo, Equivariant localization of path integrals, hep-th/9608068 [INSPIRE].

[9] V. Pestun et al., Localization techniques in quantum field theories, J. Phys. A 50 (2017) 440301 [arXiv: 1608.02952] [INSPIRE].

[10] E. Witten, Quantum Field Theory and the Jones Polynomial, Commun. Math. Phys. 121 (1989) 351 [INSPIRE]. 
[11] M. Blau and G. Thompson, Derivation of the Verlinde formula from Chern-Simons theory and the G/G model, Nucl. Phys. B 408 (1993) 345 [hep-th/9305010] [InSPIRE].

[12] M. Blau and G. Thompson, Chern-Simons theory on S1-bundles: Abelianisation and q-deformed Yang-Mills theory, JHEP 05 (2006) 003 [hep-th/0601068] [INSPIRE].

[13] C. Beasley and E. Witten, Non-Abelian localization for Chern-Simons theory, J. Diff. Geom. 70 (2005) 183 [hep-th/0503126] [INSPIRE].

[14] C. Beasley, Localization for Wilson Loops in Chern-Simons Theory, Adv. Theor. Math. Phys. 17 (2013) 1 [arXiv:0911.2687] [INSPIRE].

[15] A. Kapustin, B. Willett and I. Yaakov, Exact Results for Wilson Loops in Superconformal Chern-Simons Theories with Matter, JHEP 03 (2010) 089 [arXiv:0909.4559] [INSPIRE].

[16] A. Kapustin, B. Willett and I. Yaakov, Tests of Seiberg-like Duality in Three Dimensions, arXiv: 1012.4021 [INSPIRE].

[17] O. Aharony, Baryons, monopoles and dualities in Chern-Simons-matter theories, JHEP 02 (2016) 093 [arXiv:1512.00161] [INSPIRE].

[18] O. Aharony, A. Hanany, K.A. Intriligator, N. Seiberg and M.J. Strassler, Aspects of $N=2$ supersymmetric gauge theories in three-dimensions, Nucl. Phys. B 499 (1997) 67 [hep-th/9703110] [INSPIRE].

[19] M. Stone, Supersymmetry and the Quantum Mechanics of Spin, Nucl. Phys. B 314 (1989) 557 [INSPIRE].

[20] D. Stanford and E. Witten, Fermionic Localization of the Schwarzian Theory, JHEP 10 (2017) 008 [arXiv: 1703.04612] [INSPIRE].

[21] S. Elitzur, G.W. Moore, A. Schwimmer and N. Seiberg, Remarks on the Canonical Quantization of the Chern-Simons-Witten Theory, Nucl. Phys. B 326 (1989) 108 [InSPIRE].

[22] V. Mikhaylov and E. Witten, Branes And Supergroups, Commun. Math. Phys. 340 (2015) 699 [arXiv: 1410.1175] [INSPIRE].

[23] M. Schottenloher, A mathematical introduction to conformal field theory, Lect. Notes Phys. 759 (2008) 1.

[24] C. Closset, T.T. Dumitrescu, G. Festuccia and Z. Komargodski, Supersymmetric Field Theories on Three-Manifolds, JHEP 05 (2013) 017 [arXiv:1212.3388] [INSPIRE].

[25] C. Closset, T.T. Dumitrescu, G. Festuccia and Z. Komargodski, The Geometry of Supersymmetric Partition Functions, JHEP 01 (2014) 124 [arXiv:1309.5876] [INSPIRE].

[26] K. Ohta and Y. Yoshida, Non-Abelian Localization for Supersymmetric Yang-Mills-Chern-Simons Theories on Seifert Manifold, Phys. Rev. D 86 (2012) 105018 [arXiv: 1205.0046] [INSPIRE].

[27] R. Dijkgraaf and E. Witten, Topological Gauge Theories and Group Cohomology, Commun. Math. Phys. 129 (1990) 393 [inSPIRE].

[28] H.-C. Kao, K.-M. Lee and T. Lee, The Chern-Simons coefficient in supersymmetric Yang-Mills Chern-Simons theories, Phys. Lett. B 373 (1996) 94 [hep-th/9506170] [INSPIRE].

[29] A.N. Redlich, Gauge Noninvariance and Parity Violation of Three-Dimensional Fermions, Phys. Rev. Lett. 52 (1984) 18 [INSPIRE]. 
[30] A.N. Redlich, Parity Violation and Gauge Noninvariance of the Effective Gauge Field Action in Three-Dimensions, Phys. Rev. D 29 (1984) 2366 [INSPIRE].

[31] R.D. Pisarski and S. Rao, Topologically Massive Chromodynamics in the Perturbative Regime, Phys. Rev. D 32 (1985) 2081 [InSPIRE].

[32] M. Mariño, Chern-Simons theory, matrix integrals and perturbative three manifold invariants, Commun. Math. Phys. 253 (2004) 25 [hep-th/0207096] [INSPIRE].

[33] M. Mariño, Chern-Simons theory, matrix models and topological strings, Int. Ser. Monogr. Phys. 131 (2005) 1 [INSPIRE].

[34] F. Benini and A. Zaffaroni, A topologically twisted index for three-dimensional supersymmetric theories, JHEP 07 (2015) 127 [arXiv:1504.03698] [INSPIRE].

[35] F. Benini and A. Zaffaroni, Supersymmetric partition functions on Riemann surfaces, Proc. Symp. Pure Math. 96 (2017) 13 [arXiv:1605.06120] [INSPIRE].

[36] C. Closset and H. Kim, Comments on twisted indices in 3d supersymmetric gauge theories, JHEP 08 (2016) 059 [arXiv: 1605.06531] [INSPIRE].

[37] G.W. Moore and N. Seiberg, Taming the Conformal Zoo, Phys. Lett. B 220 (1989) 422 [INSPIRE].

[38] A. Kapustin, B. Willett and I. Yaakov, Exact results for supersymmetric abelian vortex loops in 2+1 dimensions, JHEP 06 (2013) 099 [arXiv:1211.2861] [INSPIRE].

[39] N. Drukker, T. Okuda and F. Passerini, Exact results for vortex loop operators in $3 d$ supersymmetric theories, JHEP 07 (2014) 137 [arXiv:1211.3409] [INSPIRE].

[40] K. Hosomichi, A Review on SUSY Gauge Theories on $S^{3}$, in New Dualities of Supersymmetric Gauge Theories, J. Teschner ed., pp. 307-338, Springer (2016) [DOI:10.1007/978-3-319-18769-3_10] [arXiv: 1412.7128] [INSPIRE].

[41] J. Wess and J. Bagger, Supersymmetry and Supergravity, Princeton, U.S.A., Princeton University Press (1992) [INSPIRE].

[42] G.V. Dunne, Aspects of Chern-Simons theory, in Topological Aspects of Low-dimensional Systems: Proceedings, Les Houches Summer School of Theoretical Physics, Session 69, Les Houches, France, July 7-31 1998 [hep-th/9902115] [INSPIRE].

[43] S. Elitzur, Y. Frishman, E. Rabinovici and A. Schwimmer, Origins of Global Anomalies in Quantum Mechanics, Nucl. Phys. B 273 (1986) 93 [INSPIRE].

[44] Y. Noma, Coadjoint Orbits and Wilson Loops in Five Dimensional Topological Gauge Theories, arXiv:0911.2386 [INSPIRE].

[45] E.A. Ivanov, Chern-Simons matter systems with manifest $N=2$ supersymmetry, Phys. Lett. B 268 (1991) 203 [INSPIRE].

[46] B. Willett, Localization on three-dimensional manifolds, J. Phys. A 50 (2017) 443006 [arXiv: 1608.02958] [INSPIRE].

[47] M. Aganagic, A. Klemm, M. Mariño and C. Vafa, Matrix model as a mirror of Chern-Simons theory, JHEP 02 (2004) 010 [hep-th/0211098] [INSPIRE].

[48] V. Pestun, Localization of gauge theory on a four-sphere and supersymmetric Wilson loops, Commun. Math. Phys. 313 (2012) 71 [arXiv:0712.2824] [INSPIRE]. 
[49] D. Gaiotto and X. Yin, Notes on superconformal Chern-Simons-Matter theories, JHEP 08 (2007) 056 [arXiv:0704.3740] [INSPIRE].

[50] N. Hama, K. Hosomichi and S. Lee, Notes on SUSY Gauge Theories on Three-Sphere, JHEP 03 (2011) 127 [arXiv: 1012.3512] [INSPIRE].

[51] D.L. Jafferis, The Exact Superconformal R-Symmetry Extremizes Z, JHEP 05 (2012) 159 [arXiv: 1012.3210] [INSPIRE].

[52] N. Hama, K. Hosomichi and S. Lee, SUSY Gauge Theories on Squashed Three-Spheres, JHEP 05 (2011) 014 [arXiv: 1102.4716] [INSPIRE].

[53] Y. Imamura and D. Yokoyama, $N=2$ supersymmetric theories on squashed three-sphere, Phys. Rev. D 85 (2012) 025015 [arXiv:1109.4734] [INSPIRE].

[54] A. Tanaka, Comments on knotted 1/2 BPS Wilson loops, JHEP 07 (2012) 097 [arXiv: 1204.5975] [INSPIRE].

[55] A. Kapustin and B. Willett, Wilson loops in supersymmetric Chern-Simons-matter theories and duality, arXiv:1302.2164 [INSPIRE].

[56] D. Gang, Chern-Simons theory on $L(p, q)$ lens spaces and Localization, arXiv:0912.4664 [INSPIRE].

[57] C. Closset, H. Kim and B. Willett, Supersymmetric partition functions and the three-dimensional A-twist, JHEP 03 (2017) 074 [arXiv:1701.03171] [INSPIRE].

[58] S. Sugishita and S. Terashima, Exact Results in Supersymmetric Field Theories on Manifolds with Boundaries, JHEP 11 (2013) 021 [arXiv:1308.1973] [INSPIRE].

[59] Y. Yoshida and K. Sugiyama, Localization of $3 d \mathcal{N}=2$ Supersymmetric Theories on $S^{1} \times D^{2}$, arXiv:1409.6713 [inSPIRE].

[60] S. Pasquetti, Factorisation of $N=2$ Theories on the Squashed 3-Sphere, JHEP 04 (2012) 120 [arXiv: 1111.6905] [INSPIRE].

[61] C. Beem, T. Dimofte and S. Pasquetti, Holomorphic Blocks in Three Dimensions, JHEP 12 (2014) 177 [arXiv:1211.1986] [INSPIRE].

[62] M. Fujitsuka, M. Honda and Y. Yoshida, Higgs branch localization of $3 d \mathcal{N}=2$ theories, PTEP 2014 (2014) 123B02 [arXiv:1312.3627] [INSPIRE].

[63] F. Benini and W. Peelaers, Higgs branch localization in three dimensions, JHEP 05 (2014) 030 [arXiv: 1312.6078] [INSPIRE].

[64] G. Festuccia and N. Seiberg, Rigid Supersymmetric Theories in Curved Superspace, JHEP 06 (2011) 114 [arXiv: 1105.0689] [INSPIRE].

[65] Y. Imamura and S. Yokoyama, Index for three dimensional superconformal field theories with general $R$-charge assignments, JHEP 04 (2011) 007 [arXiv: 1101.0557] [INSPIRE].

[66] L.F. Alday, D. Martelli, P. Richmond and J. Sparks, Localization on Three-Manifolds, JHEP 10 (2013) 095 [arXiv: 1307.6848] [inSPIRE].

[67] L.F. Alday, M. Fluder and J. Sparks, The Large $N$ limit of M2-branes on Lens spaces, JHEP 10 (2012) 057 [arXiv: 1204.1280] [INSPIRE].

[68] E. Guadagnini, The Link invariants of the Chern-Simons field theory: New developments in topological quantum field theory, Berlin, Germany, de Gruyter (1993), de Gruyter Expositions in Mathematics 10 (1994) [INSPIRE]. 
[69] M. Bos and V.P. Nair, Coherent State Quantization of Chern-Simons Theory, Int. J. Mod. Phys. A 5 (1990) 959 [inSPIRE].

[70] T. Dimofte, D. Gaiotto and N.M. Paquette, Dual boundary conditions in 3d SCFT's, JHEP 05 (2018) 060 [arXiv: 1712.07654] [INSPIRE].

[71] N. Drukker, D.J. Gross and H. Ooguri, Wilson loops and minimal surfaces, Phys. Rev. D 60 (1999) 125006 [hep-th/9904191] [INSPIRE].

[72] N. Seiberg and E. Witten, Gapped Boundary Phases of Topological Insulators via Weak Coupling, PTEP 2016 (2016) 12C101 [arXiv: 1602.04251] [INSPIRE].

[73] E.P. Verlinde, Fusion Rules and Modular Transformations in 2D Conformal Field Theory, Nucl. Phys. B 300 (1988) 360 [InSPIRE].

[74] M. Faizal, Y. Luo, D.J. Smith, M.-C. Tan and Q. Zhao, Gauge and supersymmetry invariance of $\mathcal{N}=2$ boundary Chern-Simons theory, Nucl. Phys. B 914 (2017) 577 [arXiv: 1601.05429] [INSPIRE].

[75] P. Di Vecchia, V.G. Knizhnik, J.L. Petersen and P. Rossi, A Supersymmetric Wess-Zumino Lagrangian in Two-Dimensions, Nucl. Phys. B 253 (1985) 701 [INSPIRE].

[76] J. Fuchs, More on the Super WZW Theory, Nucl. Phys. B 318 (1989) 631 [InSPIRE].

[77] J. Bernatska and P. Holod, Geometry and topology of coadjoint orbits of semisimple Lie groups, in Geometry, Integrability, and Quantization IX, pp. 146-166, Softex, Sofia (2008) [DOI:10.7546/giq-9-2008-146-166] [arXiv:0801.2913].

[78] B. Assel and J. Gomis, Mirror Symmetry And Loop Operators, JHEP 11 (2015) 055 [arXiv: 1506.01718] [INSPIRE].

[79] K. Intriligator and N. Seiberg, Aspects of $3 d N=2$ Chern-Simons-Matter Theories, JHEP 07 (2013) 079 [arXiv: 1305.1633] [INSPIRE].

[80] N. Doroud, J. Gomis, B. Le Floch and S. Lee, Exact Results in D $=2$ Supersymmetric Gauge Theories, JHEP 05 (2013) 093 [arXiv: 1206.2606] [INSPIRE].

[81] M. Mariño, Lectures on localization and matrix models in supersymmetric Chern-Simons-matter theories, J. Phys. A 44 (2011) 463001 [arXiv:1104.0783] [InSPIRE].

[82] E. Witten, Nonabelian Bosonization in Two-Dimensions, Commun. Math. Phys. 92 (1984) 455 [INSPIRE].

[83] P. Di Francesco, P. Mathieu and D. Sénéchal, Conformal Field Theory, Graduate Texts in Contemporary Physics, Springer-Verlag, New York (1997) [DOI:10.1007/978-1-4612-2256-9].

[84] K. Hori et al., Mirror Symmetry, AMS, Providence, U.S.A. (2003) [INSPIRE]. 